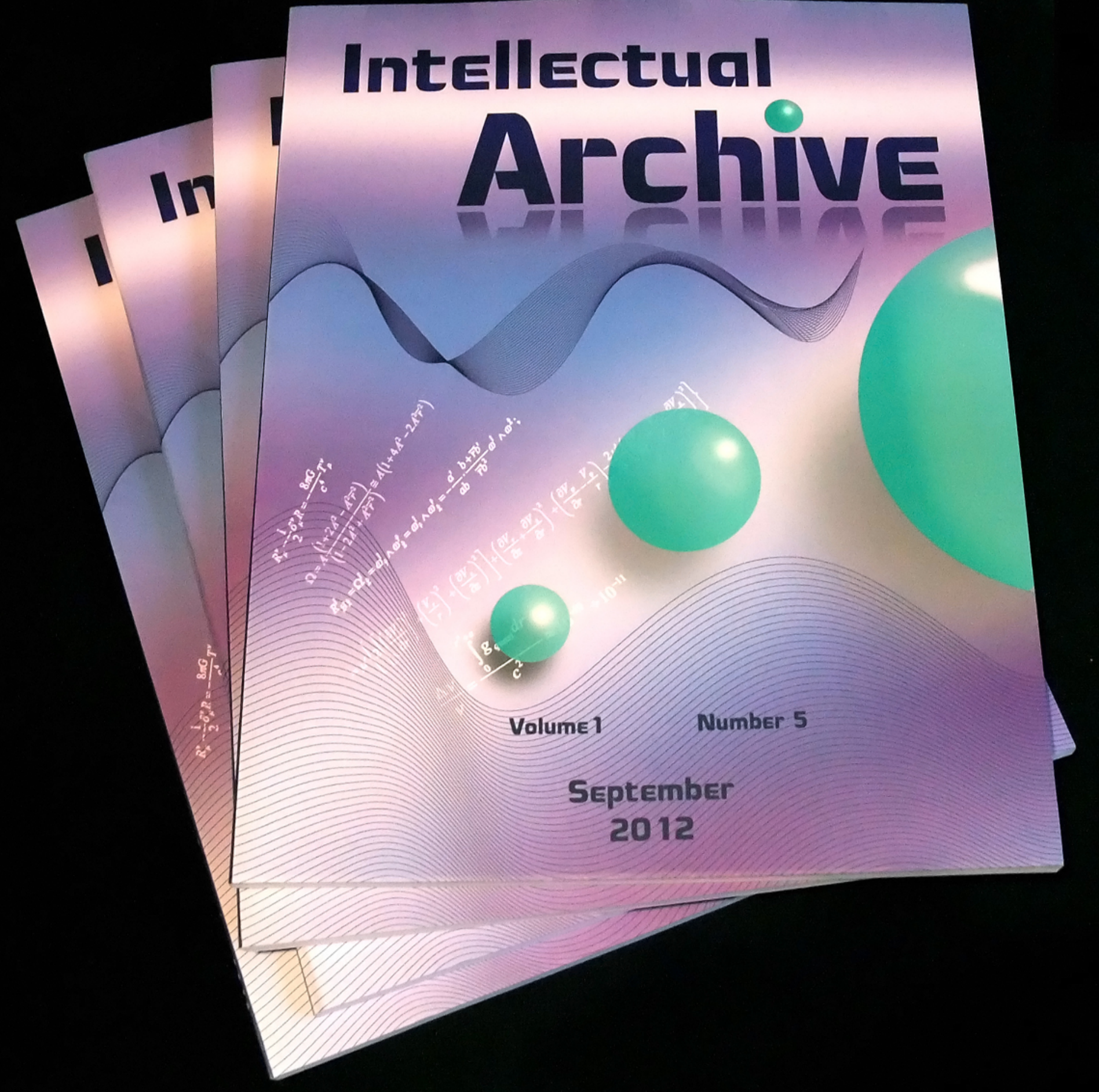




\section{Intel|ectua|}
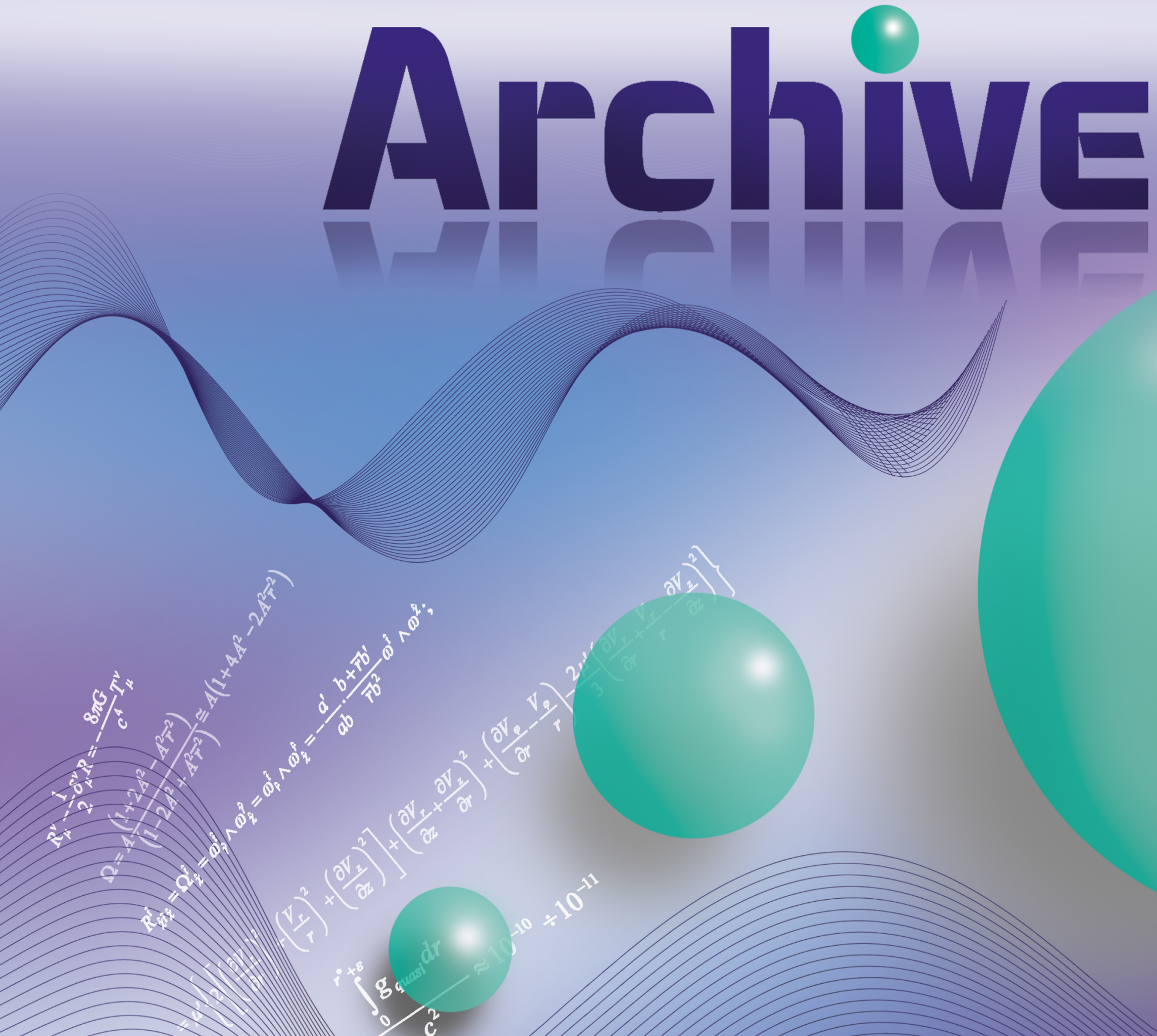


\section{IntellectualArchive}

Volume 1, Number 5

$\begin{array}{ll}\text { Publisher } & \text { : Shiny World Corp. } \\ \text { Address } & : 9350 \text { Yonge Street } \\ & \text { P.O.Box 61533, } \\ & \text { Richmond Hill, Ontario } \\ & \text { L4C 3N0 } \\ & \text { Canada }\end{array}$

E-mail : support@IntellectualArchive.com

Web Site : www.IntellectualArchive.com

Series : Journal

Frequency : Monthly

Month : : September of 2012

ISSN : :1929-4700

Trademark : IntellectualArchive ${ }^{\text {TM }}$

(C) 2012 Shiny World Corp. All Rights Reserved. No reproduction allowed without permission. Copyright and moral rights of all articles belong to the individual authors. 


\section{Intellectual \\ Archive}

Volume 1

Alexander $\mathrm{L}$.

Dmitriev

Daniele Sasso

Alexander Krasulin
Number 5

\section{Table of Contents}

\section{Astronomy}

Tidal interactions and principle of corresponding states: from micro to macro cosmos. A century after van der Waals' Nobel Prize

\section{Physics}

Weight Of The Thermo-Insulated Container Equipped With Electric Heater

Relativistic Theory of Black Holes

\section{Mathematics}

Five-Dimensional Tangent Vectors in Space-Time IV. Generalization of Exterior Calculus

\section{Computer Science}

G.A. Tarnavsky, E.V. Computer Simulation Center in Internet Vorozhtsov

\section{Biology}

J.C. Hodge

How are white squirrels able to survive in a forest?

\section{Economics}

Customers' Motivation as the Basis of Effective Forming of

Communication Mix

\section{Linguistics}

A Cognitive Study on Conceptual Metaphors of Emotion (English / Russian)

\section{Education}

Baisheva M.I.

Semantic development of preschool children

Ecological education of pre-school children in developing environment

Nikolaeva L.V. 


\title{
Tidal interactions and principle of corresponding states: from micro to macro cosmos. A century after van der Waals' Nobel Prize
}

\author{
R. Caimmi*
}

October 2, 2012

\begin{abstract}
The current attempt is aimed to honor the first centennial of Johannes Diderik van der Waals (VDW) awarding Nobel Prize in Physics. The VDW theory of ordinary fluids is reviewed in the first part of the paper, where special effort is devoted to the equation of state and the law of corresponding states. In addition, a few mathematical features involving properties of cubic equations are discussed, for appreciating the intrinsic beauty of the VDW theory. A theory of astrophysical fluids is shortly reviewed in the second part of the paper, grounding on the tensor virial theorem for two-component systems, and an equation of state is formulated with a convenient choice of reduced variables. Additional effort is devoted to particular choices of density profiles, namely a simple guidance case and two cases of astrophysical interest. The related macroisothermal curves are found to be qualitatively similar to VDW isothermal curves below the critical threshold and, for sufficiently steep density profiles, a critical macroisothermal curve exists, with a single horisontal inflexion point. Under the working hypothesis of a phase transition (assumed to be gas-stars) for astrophysical fluids, similar to the vapour-liquid
\end{abstract}

* Physics and Astronomy Department, Padua Univ., Vicolo Osservatorio 3/2, I-35122 Padova, Italy email: roberto.caimmi@unipd.it fax: 39-049-8278212 
phase transition in ordinary fluids, the location of gas clouds, stellar systems, galaxies, cluster of galaxies, on the plane scanned by reduced variables, is tentatively assigned. A brief discussion shows how van der Waals' two great discoveries, namely a gas equation of state where tidal interactions between molecules are taken into account, and the law of corresponding states, related to microcosmos, find a counterpart with regard to macrocosmos. In conclusion, after a century since the awarding of the Nobel Prize in Physics, van der Waals' ideas are still valid and helpful to day for a full understanding of the universe. keywords - galaxies: evolution - dark matter: haloes.

\section{Introduction}

One century ago (1910), the Nobel Prize in Physics was awarded to Johannes Diderik van der Waals (hereafter quoted as VDW). In his doctoral thesis (1873) the ideal gas equation of state was generalized for embracing both the gaseous and the liquid state, where these two states of aggregation not only merge into each other in a continuous manner, but are in fact of the same nature. With respect to ideal gases, the volume of the molecules and the intermolecular tidal forces were taken into account.

The VDW equation was later reformulated in terms of reduced (dimensionless) variables (1880), which allows the description of all substances in terms of a single equation. In other words, the state of any substance, defined by the values of reduced volume, reduced pressure, and reduced temperature, is independent of the nature of the substance. This result is known as the law of corresponding states.

The VDW equation of state in dimensional and reduced form, served as a guide during experiments which ultimately led to hydrogen (1898) and helium (1908) liquefaction. The Cryogenic Laboratory at Leiden had developed under the influence of VDW's theories. For further details on VDW's biography refer to specialized textbooks (e.g., Nobel Lectures 1967).

The current paper has been written in honor of the first centennial of VDW awarding Nobel Prize in Physics. The ideal and VDW equation of state, both in dimensional and reduced form, are reviewed, and a number of features are analysed in detail, in Section 2. Counterparts to ideal and VDW equations of state for astrophysical fluids, or macrogases, are briefly summarized and compared with the classical formulation in Section 3. The discussion and the conclusion are drawn in Section 4. 


\section{Equation of state of ordinary fluids}

Let ordinary fluids be conceived as fluids which can be investigated in laboratory. The simplest description is provided by the theory of ideal gas, where the following restrictive assumptions are made: (i) particles are identical spheres; (ii) the number of particles is extremely large; (iii) the motion of particles is random; (iv) collisions between particles or with the wall of the box are perfectly elastic; (v) interactions between particles or with the wall of the box are null.

The equation of state of ideal gases may be written under the form (e.g., Landau and Lifchitz, 1967, Chap. IV, §42, hereafter quoted as LL67):

$$
p V=k N T \text {; }
$$

where $p$ is the pressure, $V$ the volume, $T$ the temperature, $N$ the particle number, and $k$ the Boltzmann constant.

In getting a better description of ordinary fluids, the above assumption (v) is relaxed and tidal interactions between particles are taken into consideration. The VDW generalization of the equation of state of ideal gases, Eq. (1), reads (van der Waals, 1873):

$$
\left(p+A \frac{N^{2}}{V^{2}}\right)(V-N B)=k N T ;
$$

where $A$ and $B$ are constants which depend on the nature of the particles. More specifically, the presence of an attractive interaction between particles reduces both the force and the frequency of particle-wall collisions: the net effect is a reduction of the pressure, proportional to the square numerical density, expressed as $A(N / V)^{2}$. On the other hand, the whole volume of the box, $V$, is not accessible to particles, in that they are conceived as identical spheres: the free volume within the box is $V-N B$, where $B$ is the volume of a single sphere. For further details refer to specific textbooks (e.g., LL67, Chap. VII, §74).

The isothermal ( $T=$ const) curves for ideal gases are hyperbolas with axes, $p=\mp V$, conformly to Eq. (1). In VDW theory of real gases, the isothermal curves exhibit two extremum points below a threshold, which reduce to a single horisontal inflexion point when a critical temperature is attained, as shown in Fig. 1. Well above the critical isothermal curve, $T \gg T_{\mathrm{c}}$, the trends exhibited by ideal and VDW gases look very similar. Below the critical isothermal curve, $T<T_{\mathrm{c}}$, the behaviour of VDW gases is different with respect to ideal gases and, in addition, the related isothermal curves provide a wrong description within a specific region where saturated vapour and liquid phases coexist. Further details are shown in Fig. 2. Above the critical 

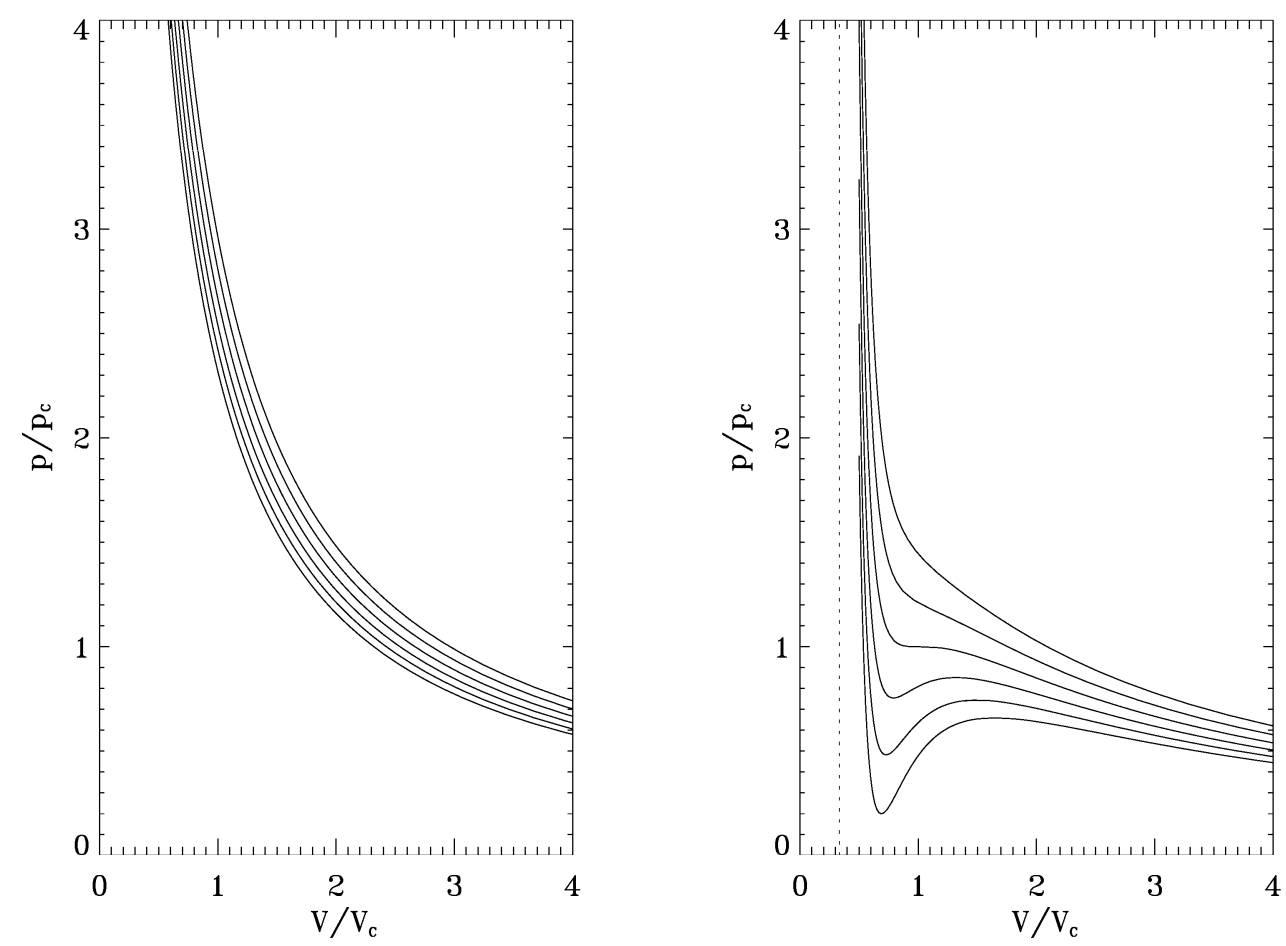

Figure 1: Isothermal curves related to ideal (left panel) and VDW (right panel) gases, respectively. Isothermal curves (from bottom to top) correspond to $T / T_{\mathrm{c}}=20 / 23,20 / 22,20 / 21,20 / 20,20 / 19,20 / 18$. No extremum point exists above the critical isothermal curve, $T / T_{\mathrm{c}}=1$.

isothermal curve $\left(T=T_{\mathrm{c}}\right)$ the trend is similar with respect to ideal gases. Below the critical isothermal curve and on the right of the dashed curve, the supersaturated vapour still behaves as an ideal gas. Below the critical isothermal curve and on the left of the dashed curve, the liquid shows little change in volume as the pressure rises. Within the bell-shaped area bounded by the dashed curve, the liquid phase is in equilibrium with the saturated vapour phase. A diminished volume implies smaller saturated vapour fraction and larger liquid fraction at constant pressure, and vice versa. The VDW equation of state is no longer valid in this region. The dashed curve (including the central branch) is the locus of intersections between VDW and real isothermal curves, the latter being related to constant pressure where liquid and vapour phases coexist. The dotted curve is the locus of VDW isothermal extremum points. 
A specific $\left(T / T_{\mathrm{c}}=20 / 23\right) \mathrm{VDW}$ and corresponding real isothermal curve, are represented in Fig. 3. The VDW isothermal curve and the real isothermal curve coincide within the range, $V \leq V_{\mathrm{A}}$ and $V \geq V_{\mathrm{E}}$. The VDW isothermal curve exhibits two extremum points: a minimum, $B$, and a maximum, $\mathrm{D}$, while the real isothermal curve is flat, within the range, $V_{\mathrm{A}} \leq V \leq V_{\mathrm{E}}$. Configurations related to the VDW isothermal curve within the range, $V_{\mathrm{A}} \leq$ $V \leq V_{\mathrm{B}}$ (due to tension forces acting on the particles yielding superheated liquid), and $V_{\mathrm{D}} \leq V \leq V_{\mathrm{E}}$ (due to the occurrence of undercooled vapour), may be obtained under special conditions, while configurations within the range, $V_{\mathrm{B}} \leq V \leq V_{\mathrm{D}}$, are always unstable. The volumes, $V_{\mathrm{A}}$ and $V_{\mathrm{E}}$, correspond to the maximum value in presence of the sole liquid phase and the minimum value in presence of the sole vapour phase, respectively.

The surfaces, $A B C$ and $C D E$, are equal, as first inferred by Maxwell (e.g., Rostagni, 1957, Chap.XII, §19). The VDW and real isothermal curves represented in Fig. 3 being related to the same temperature, $T$, the cycle, ABCDECA, is completely both isothermal and reversible, and the work, $W$, performed therein cannot be positive to avoid violation of the second law of the thermodynamics. The cycles, ABCA and CDEC, occurring in counterclockwise and clockwise sense, respectively, are also completely both isothermal and reversible. Accordingly, $W_{\mathrm{ABCDECA}}=W_{\mathrm{ABCA}}-W_{\mathrm{CDEC}} \leq 0$. A similar procedure, related to the reversed cycle, ACEDCBA, yields $W_{\text {ACEDCBA }}=$ $W_{\text {CEDC }}-W_{\text {CBAC }} \leq 0$. Then $W_{\text {ABCDECA }}=W_{\text {ACEDCBA }}=0$, which implies $W_{\mathrm{ABCA}}=W_{\mathrm{CDEC}}=W_{\mathrm{CEDC}}=W_{\mathrm{CBAC}}$ and, in turn, the equality between the related surfaces. For further details refer to specific textbooks (e.g., LL67. Chap. VIII, §85).

In order to simplify both notation and calculations, it is convenient to deal with (dimensionless) reduced variables (e.g., Rostagni, 1957, Chap. XII, $\S 16$; LL67, Chap. VIII, $\S 85)$. To this aim, the first step is the knowledge of the parameters related to the critical point, $V_{\mathrm{c}}, p_{\mathrm{c}}, T_{\mathrm{c}}$. Using the VDW equation of state, Eq. (2), the pressure and its first and second partial derivatives, with respect to the volume, read:

$$
\begin{aligned}
& p=\frac{k N T}{V-N B}-A \frac{N^{2}}{V^{2}} ; \quad N=\text { const } ; \\
& \left(\frac{\partial p}{\partial V}\right)_{V, T}=-\frac{k N T}{(V-N B)^{2}}+2 A \frac{N^{2}}{V^{3}} ; \\
& \left(\frac{\partial^{2} p}{\partial V^{2}}\right)_{V, T}=\frac{2 k N T}{(V-N B)^{3}}-6 A \frac{N^{2}}{V^{4}} ;
\end{aligned}
$$

where the domain is $V>N B, V=N B$ is a vertical asymptote, and $p=0$ is a horisontal asymptote. The critical isothermal corresponds to the highest 
temperature allowing a liquid phase, which occurs therein only at the critical point. The critical isothermal curve exhibits neither a minimum nor a maximum, which are replaced by a horisontal inflexion point coinciding with the critical point. Accordingly, $(\partial p / \partial V)_{V_{\mathrm{c}}, T_{\mathrm{c}}}=0,\left(\partial^{2} p / \partial V^{2}\right)_{V_{\mathrm{c}}, T_{\mathrm{c}}}=0$, and $p_{\mathrm{c}}=k N T_{\mathrm{c}} /\left(V_{\mathrm{c}}-N B\right)-A N^{2} / V_{\mathrm{c}}^{2}$. The solution of the related system is:

$$
\begin{aligned}
& V_{\mathrm{c}}=3 N B ; \\
& T_{\mathrm{c}}=\frac{8}{27} \frac{A}{B} \frac{1}{k} ; \\
& p_{\mathrm{c}}=\frac{1}{27} \frac{A}{B^{2}} ; \\
& Z_{c}=\frac{p_{\mathrm{c}} V_{\mathrm{c}}}{k N T_{\mathrm{c}}}=\frac{3}{8} ;
\end{aligned}
$$

where, in general, the compressibility factor, $Z=p V /(k N T)$, defines the degree of departure from the behaviour of ideal gases, for which $Z=1$, according to Eq. (1). For further details refer to specific textbooks (e.g., Rostagni, 1957, Chap. XII, §20; LL67, Chap. VIII, §85).

With regard to the reduced variables:

$$
Y=\frac{V}{V_{\mathrm{c}}} ; \quad \not p=\frac{p}{p_{\mathrm{c}}} ; \quad T=\frac{T}{T_{\mathrm{c}}} ;
$$

the ideal gas equation of state, Eq.(1), and the VDW equation of state, Eq. (2), reduce to:

$$
\begin{aligned}
& \not p V=\frac{8}{3} T ; \\
& \left(\not p+\frac{3}{Y^{2}}\right)\left(Y-\frac{1}{3}\right)=\frac{8}{3} T ; \quad Y>\frac{1}{3} ;
\end{aligned}
$$

and Eqs. (3), (4), and (5), reduce to:

$$
\begin{aligned}
& \not p=\frac{8 T}{3 Y-1}-\frac{3}{V^{2}} ; \\
& \left(\frac{\partial \not p}{\partial Y}\right)_{Y, T}=-\frac{24 T}{(3 Y-1)^{2}}+\frac{6}{Y^{3}} ; \\
& \left(\frac{\partial^{2} \not p}{\partial Y^{2}}\right)_{Y, T}=\frac{144 T}{(3 Y-1)^{3}}-\frac{18}{Y^{4}} ;
\end{aligned}
$$

where, for assigned $T$, the domain of the function, $\not p(V)$, is $Y>1 / 3, Y=$ $1 / 3$ is a vertical asymptote, and $\not p=0$ is a horisontal asymptote. In the 
special case of the critical point, $Y=1, T=1, \not p=1$, the partial derivatives are null, as expected.

The extremum points, via Eq. (14), are defined by the relation:

$$
f(Y)=\frac{(3 Y-1)^{2}}{4 V^{3}}=T
$$

which is satisfied at the critical point, as expected. The function on the left-hand side of Eq. (16) has two extremum points: a minimum at $Y=1 / 3$ (outside the physical domain) and a maximum at $V=1$, where $T=1$. Accordingly, Eq. (16) is never satisfied for $T>1$, which implies no extremum point for related isothermal curves, as expected. The contrary holds for $T<1$, where it can be seen that the third-degree equation associated to Eq. (14) has three real solutions, related to extremum points. One lies outside the physical domain, which implies $Y \leq 1 / 3$. The remaining two are obtained as the intersections between the curve, $f(Y)$, expressed by Eq. (16), and the straight line, $y=T$, keeping in mind that $f(1 / 3)=0, f(1)=1$, and $\lim _{Y \rightarrow+\infty} f(Y)=0$.

The third-degree equation associated to Eq. (14), may be ordered as:

$$
\begin{aligned}
& Y^{3}-9 a V^{2}+6 a Y-a=0 ; \\
& a=\frac{1}{4 T} ;
\end{aligned}
$$

with regard to the standard formulation (e.g., Spiegel, 1968, Chap. 9):

$$
x^{3}+a_{1} x^{2}+a_{2} x+a_{3}=0 ;
$$

the discriminants of Eq. (17a) are:

$$
\begin{aligned}
& Q=\frac{3 a_{2}-a_{1}^{2}}{9}=a(2-9 a) ; \\
& R=\frac{9 a_{1} a_{2}-27 a_{3}-2 a_{1}^{3}}{54}=\frac{a\left(1-18 a+54 a^{2}\right)}{2} ; \\
& D=Q^{3}+R^{2}=\frac{a^{2}(1-4 a)}{4} ;
\end{aligned}
$$

where $D=0$ in the special case of the critical isothermal curve $(T=1, a=$ 1/4), $D<0$ for $T<1$, and $D>0$ for $T>1$. Accordingly, three coincident real solutions exist if $D=0$, three (at least two) different real solutions if $D<0$, one real (outside the physical domain) and two complex coniugate if $D>0$. 
The three real solutions $(D \leq 0)$ may be expressed as (e.g., Spiegel, 1968, Chap. 9):

$$
\begin{aligned}
& Y_{1}=2 \sqrt{-Q} \cos \left(\pi+\frac{\theta}{3}\right)-\frac{1}{3} a_{1} ; \\
& Y_{2}=2 \sqrt{-Q} \cos \left(\pi+\frac{\theta}{3}+\frac{2 \pi}{3}\right)-\frac{1}{3} a_{1} ; \\
& Y_{3}=2 \sqrt{-Q} \cos \left(\pi+\frac{\theta}{3}+\frac{4 \pi}{3}\right)-\frac{1}{3} a_{1} ; \\
& \theta=\arctan \frac{\sqrt{-D}}{R}
\end{aligned}
$$

where $a_{1}=-9 a$ and, in the special case of the critical isothermal curve, $a=1 / 4, Q=-1 / 16, D=0$, which implies $V_{0}=\min \left(V_{1}, V_{2}, Y_{3}\right), Y_{\mathrm{A}}=V_{\mathrm{B}}=$ $V_{\mathrm{C}}=V_{\mathrm{D}}=V_{\mathrm{E}}=\max \left(V_{1}, Y_{2}, V_{3}\right)$. In the special case, $T \rightarrow 0$, Eq. (17a) reduces to a second-degree equation whose solutions are $Y_{01}=Y_{02}=1 / 3$, while the related function is otherwise divergent as $a \rightarrow+\infty$. In general, the extremum points of VDW isothermal curves $(T \leq 1)$ occur at $Y=V_{\mathrm{B}}$ (minimum) and $Y=V_{\mathrm{D}}$ (maximum), $V_{\mathrm{B}} \leq V_{\mathrm{D}}$. As $T \rightarrow 0, V_{\mathrm{B}} \rightarrow 1 / 3$, $V_{\mathrm{D}} \rightarrow+\infty$, where, in all cases, $1 / 3<Y_{\mathrm{B}} \leq 1 \leq Y_{\mathrm{D}}$.

The two areas defined by the intersection of a generic VDW isothermal curve $(T \leq 1)$ and related real isothermal curves (see Fig. 3), are expressed as:

$$
\begin{aligned}
& W_{1}=\int_{V_{\mathrm{A}}}^{V_{\mathrm{C}}} p_{\mathrm{C}} \mathrm{d} V-\int_{V_{\mathrm{A}}}^{V_{\mathrm{C}}} p \mathrm{~d} V=p_{\mathrm{C}} V_{\mathrm{C}}\left[\not p_{C}\left(V_{\mathrm{C}}-Y_{\mathrm{A}}\right)-\int_{Y_{\mathrm{A}}}^{V_{\mathrm{C}}} \not p \mathrm{~d} V\right] \\
& W_{2}=\int_{V_{\mathrm{C}}}^{V_{\mathrm{E}}} p \mathrm{~d} V-\int_{V_{\mathrm{C}}}^{V_{\mathrm{E}}} p_{\mathrm{C}} \mathrm{d} V=p_{\mathrm{C}} V_{\mathrm{C}}\left[\int_{V_{\mathrm{C}}}^{V_{\mathrm{E}}} \not p \mathrm{~d} V-\not p_{C}\left(V_{\mathrm{E}}-V_{\mathrm{C}}\right)\right]
\end{aligned}
$$

and the substitution of Eq. (13) into (23) allows explicit expressions for the integrals. The result is:

$$
\begin{aligned}
& \frac{W_{1}}{p_{\mathrm{C}} V_{\mathrm{C}}}=\not \phi_{\mathrm{C}}\left(V_{\mathrm{C}}-V_{\mathrm{A}}\right)-\frac{8}{3} T \ln \frac{3 V_{\mathrm{C}}-1}{3 V_{\mathrm{A}}-1}+\frac{3\left(V_{\mathrm{C}}-V_{\mathrm{A}}\right)}{V_{\mathrm{A}} V_{\mathrm{C}}} ; \\
& \frac{W_{2}}{p_{\mathrm{C}} V_{\mathrm{C}}}=\frac{8}{3} T \ln \frac{3 Y_{\mathrm{E}}-1}{3 V_{\mathrm{C}}-1}-\frac{3\left(V_{\mathrm{E}}-V_{\mathrm{C}}\right)}{V_{\mathrm{C}} V_{\mathrm{E}}}-\not p_{C}\left(V_{\mathrm{E}}-Y_{\mathrm{C}}\right) ;
\end{aligned}
$$

and the condition, $W_{1}=W_{2}$, after some algebra reads (Caimmi 2010, hereafter quoted as C10):

$$
\not \not_{C}=\frac{8}{3} \frac{T}{V_{\mathrm{E}}-V_{\mathrm{A}}} \ln \frac{3 Y_{\mathrm{E}}-1}{3 V_{\mathrm{A}}-1}-\frac{3}{V_{\mathrm{A}} V_{\mathrm{E}}} ;
$$


where, for a selected isothermal curve, the unknowns are $\not p_{C}=\not p_{A}=\not p_{E}, V_{\mathrm{A}}$; and $V_{\mathrm{E}}$.

The reduced volumes, $Y_{\mathrm{A}}, Y_{\mathrm{C}}, Y_{\mathrm{E}}$, see Fig. 3, may be considered as intersections between a VDW isothermal curve $(T<1)$ and a horisontal straight line, $\not p=\not p C$, in the $(\mathrm{O} Y \not p)$ plane. In other words, $V_{\mathrm{A}}, V_{\mathrm{C}}, V_{\mathrm{E}}$, are the real solutions of the third-degree equation:

$$
V^{3}-\left(\frac{1}{3}+\frac{8}{3} \frac{T}{\not p_{C}}\right) V^{2}+\frac{3}{\not p_{C}} Y-\frac{1}{\not p_{C}}=0 ;
$$

which has been deduced from Eq. (13), particularized to $\not p=\not p \phi_{C}$. The related solutions may be calculated using Eqs. (22). The last unknown, $\not p_{C}$, is determined from Eq. (25).

An inspection of Fig. 3 shows that the points, A and E, are located on the left of the minimum, B, and on the right of the maximum, D, respectively. Keeping in mind the above results, the following inequality holds: $V_{\mathrm{A}} \leq$ $V_{\mathrm{B}} \leq 1 \leq V_{\mathrm{D}} \leq V_{\mathrm{E}}$, which implies further investigation on the special case, $V_{\mathrm{C}}=1$. The particularization of the VDW equation of state, Eq. (13), to the point, $\mathrm{C}=\mathrm{C}_{1}$, assuming $\mathrm{Y}_{C_{1}}=1$, yields:

$$
T=\frac{\not C_{C_{1}}+3}{4}
$$

and Eq. (26) reduces to:

$$
\begin{aligned}
& Y^{3}-(1+2 b) Y^{2}+3 b Y-b=0 ; \\
& b=\frac{1}{\not p_{C_{1}}} ;
\end{aligned}
$$

with regard to the generic third-degree equation, Eq. (18), the three solutions, $x_{1}, x_{2}, x_{3}$, satisfy the relations (e.g., Spiegel, 1968, Chap. 9):

$$
\begin{aligned}
& x_{1}+x_{2}+x_{3}=-a_{1} ; \\
& x_{1} x_{2}+x_{2} x_{3}+x_{3} x_{1}=a_{2} ; \\
& x_{1} x_{2} x_{3}=-a_{3} ;
\end{aligned}
$$

where, in the case under discussion:

$$
\begin{array}{ll}
a_{1}=-1-2 b ; \quad a_{2}=3 b ; & a_{3}=-b ; \\
x_{1}=V_{\mathrm{A}} ; \quad x_{2}=V_{C_{1}}=1 ; & x_{3}=V_{\mathrm{E}} ;
\end{array}
$$

and the substitution of Eqs. (30) into two among (29) yields:

$$
\begin{aligned}
& V_{\mathrm{A}}=b-\sqrt{b^{2}-b} \\
& Y_{\mathrm{E}}=b+\sqrt{b^{2}-b}
\end{aligned}
$$


and the combination of Eqs. (27), (28b), and (31) produces:

$$
\begin{array}{ll}
Y_{\mathrm{A}}=\frac{1-2 \sqrt{1-T}}{4 T-3} ; & T \leq 1 ; \\
Y_{\mathrm{E}}=\frac{1+2 \sqrt{1-T}}{4 T-3} ; & T \leq 1 ; ;
\end{array}
$$

which, together with $V_{C_{1}}=1$, are the abscissae of the intersection points between a selected VDW isothermal curve in the $(\mathrm{O} Y \not p)$ plane and the straight line, $\not p=\not \phi_{C_{1}}$, in the special case under discussion.

The substitution of Eqs. (32) into (25), the last being related to the real isothermal curve, yields:

$$
\frac{T}{\sqrt{1-T}} \ln \frac{3-2 T+3 \sqrt{1-T}}{3-2 T-3 \sqrt{1-T}}=6 ;
$$

which holds only for the critical isothermal curve, $T=1$. Accordingly, the abscissa of the intersection point, $\mathrm{C}$, between a selected VDW isothermal curve and related real isothermal curve, see Fig. 3, cannot occur at $V_{\mathrm{C}}=1$ unless the critical isothermal curve is considered. Then the third-degree equation, Eq. (26), must be solved in the general case by use of Eqs. (22). The results are shown in Tab. 1, where the following parameters (in reduced variables) are listed for each VDW isothermal curve, see Fig. 3: the temperature, $T$, the lower volume limit, $V_{\mathrm{A}}$, for which the liquid and vapour phase coexist; the extremum point (minimum) volume, $V_{B}$; the intermediate volume, $V_{C}$, for which the pressure equals its counterpart related to the corresponding lower and upper volume limit, for which the liquid and vapour phase coexist; the extremum point (maximum) volume, $Y_{D}$; the upper volume limit, $V_{E}$, for which the liquid and vapour phase coexist; the extremum point (minimum) pressure, $\not p_{B}$; the pressure, $\not \not_{A}=\not \not_{C}=\not p_{E}$, related to the horisontal real isothermal curve; the extremum point (maximum) pressure, $\not p_{D}$. The locus of the intersections between VDW and real isothermal curves is represented in Fig. 2 as a trifid curve, where the left, the right, and the middle branch correspond to $Y_{\mathrm{A}}, V_{\mathrm{E}}$, and $V_{\mathrm{C}}$, respectively. The common starting point coincides with the critical point. The locus of the VDW isothermal curve extremum points is represented in Fig. 2 as a dotted curve starting from the critical point, where the left and the right branch corresponds to minimum and maximum points, respectively.

A fluid state can be represented in reduced variables as $(Y, \not p, T)$, where one variable may be expressed as a function of the remaining two, by use 
Table 1: Values of parameters, $\not, Y_{\mathrm{A}}, Y_{\mathrm{B}}, V_{\mathrm{C}}, Y_{\mathrm{D}}, Y_{\mathrm{E}}, \not p_{B}, \not p_{C}, \not p_{D}$, within the range, $0.85 \leq T \leq 0.99$, using a step, $\Delta T=0.01$. Additional values are computed near the critical point, to increase the resolution. The true value of the reduced temperature on the last row is $T=0.9999$ or $10 T=9.999$. All values equal unity at the critical point. Index captions: $\mathrm{A}, \mathrm{C}, \mathrm{E}$ - intersections between VDW and real isothermal curves; B - extremum point of minimum; D - extremum point of maximum. Extremum points are related to VDW isothermal curves, while their real counterparts are flat in presence of both liquid and vapour phase. To save aesthetics, 01 on head columns stands for unity.

\begin{tabular}{|l|l|l|l|l|l|l|l|l|}
\hline $10 T$ & $10 \bigvee_{\mathrm{A}}$ & $10 \bigvee_{\mathrm{B}}$ & $01 \bigvee_{\mathrm{C}}$ & $01 \bigvee_{\mathrm{D}}$ & $01 \bigvee_{\mathrm{E}}$ & $10 \not p_{B}$ & $10 \not \not_{C}$ & $10 \not p_{D}$ \\
\hline 8.50 & 5.5336 & 6.7168 & 1.1453 & 1.7209 & 3.1276 & 0.4963 & 5.0449 & 6.2055 \\
8.60 & 5.6195 & 6.8003 & 1.1337 & 1.6821 & 2.9545 & 1.2750 & 5.3125 & 6.4005 \\
8.70 & 5.7116 & 6.8883 & 1.1225 & 1.6436 & 2.7909 & 2.0346 & 5.5887 & 6.6011 \\
8.80 & 5.8106 & 6.9814 & 1.1116 & 1.6052 & 2.6360 & 2.7752 & 5.8736 & 6.8076 \\
8.90 & 5.9176 & 7.0804 & 1.1009 & 1.5669 & 2.4889 & 3.4965 & 6.1674 & 7.0205 \\
9.00 & 6.0340 & 7.1860 & 1.0905 & 1.5285 & 2.3488 & 4.1984 & 6.4700 & 7.2401 \\
9.10 & 6.1615 & 7.2994 & 1.0804 & 1.4900 & 2.2151 & 4.8807 & 6.7816 & 7.4669 \\
9.20 & 6.3022 & 7.4221 & 1.0706 & 1.4511 & 2.0869 & 5.5430 & 7.1021 & 7.7014 \\
9.30 & 6.4593 & 7.5561 & 1.0610 & 1.4117 & 1.9634 & 6.1849 & 7.4318 & 7.9443 \\
9.40 & 6.6369 & 7.7040 & 1.0516 & 1.3715 & 1.8438 & 6.8058 & 7.7707 & 8.1963 \\
9.50 & 6.8412 & 7.8697 & 1.0425 & 1.3300 & 1.7271 & 7.4049 & 8.1188 & 8.4584 \\
9.60 & 7.0819 & 8.0593 & 1.0336 & 1.2867 & 1.6118 & 7.9811 & 8.4762 & 8.7319 \\
9.70 & 7.3756 & 8.2830 & 1.0249 & 1.2404 & 1.4960 & 8.5328 & 8.8429 & 9.0185 \\
9.80 & 7.7554 & 8.5611 & 1.0164 & 1.1892 & 1.3761 & 9.0576 & 9.2191 & 9.3209 \\
9.90 & 8.3091 & 8.9461 & 1.0081 & 1.1278 & 1.2430 & 9.5510 & 9.6048 & 9.6437 \\
9.95 & 8.7471 & 9.2353 & 1.0040 & 1.0876 & 1.1618 & 9.7830 & 9.8012 & 9.8157 \\
9.98 & 9.1727 & 9.5049 & 1.0016 & 1.0540 & 1.0972 & 9.9158 & 9.9202 & 9.9240 \\
9.99 & 9.4018 & 9.6456 & 1.0008 & 1.0377 & 1.0670 & 9.9585 & 9.9600 & 9.9614 \\
9.99 & 9.8035 & 9.8856 & 1.0001 & 1.0117 & 1.0204 & 9.9960 & 9.9960 & 9.9960 \\
\hline
\end{tabular}


of the reduced ideal gas equation of state, Eq. (11), or the reduced VDW equation of state, Eq. (12). The formulation in terms of reduced variables, Eqs. (10), makes the related equation of state universal i.e. it holds for any fluid. Similarly, the Lane-Emden equation expressed in polytropic (dimensionless) variables, describes the whole class of polytropic gas spheres with assigned polytropic index, in hydrostatic equilibrium (e.g., Chandrasekhar 1939, Chap. IV, §4).

The states of two fluids with equal $(Y, \not p, T)$, are defined as corresponding states. The mere existence of an equation of state yields the following result.

Law of corresponding states. Given two fluids, the equality between two among three reduced variables, $Y, \not p, T$, implies the equality between the remaining related reduced variables i.e. the two fluids are in corresponding states.

The law was first formulated by van der Waals in 1880. For further details refer to specific textbooks (e.g., LL67, Chap. VIII, §85).

\section{Equation of state of astrophysical fluids}

Let macrogases be defined as two-component fluids which interact only gravitationally. For assigned density profiles, the virial theorem can be formulated for each subsystem, where the potential energy is the sum of the self potential energy of the component under consideration, and the tidal energy induced by the other one. The virial theorem for subsystem can be expressed as a macrogas equation of state in terms of dimensionless variables, $X_{V}, X_{p}, X_{T}$; related to axis ratio, mass ratio, virial (i.e. self + tidal) potential energy ratio, respectively. The result is $(\mathrm{C} 10)$ :

$$
\begin{aligned}
& X_{p} X_{V} F_{X}\left(X_{p}, X_{V}\right)=X_{T} ; \\
& X_{p}=m^{2} ; \quad X_{V}=\frac{1}{y} ; \quad X_{T}=\phi ;
\end{aligned}
$$

where the function, $F_{X}$, depends on the selected density profiles, $m$ is the (outer to inner component) mass ratio, $y$ is the (outer to inner component) axis ratio along a generic direction, $\phi$ is the (outer to inner component) virial energy ratio, and the density profiles are restricted to be homeoidally striated. The variables, $X_{V}, X_{p}, X_{T}$, play a similar role as the volume, the pressure, and the temperature, for ordinary fluids. Accordingly, $X_{V}, X_{p}$; $X_{T}$, may be defined as macrovolume, macropressure, and macrotemperature, respectively. For further details refer to the parent paper (C10). 
Macroisothermal curves on the $\left(\mathrm{O} X_{V} X_{p}\right)$ plane exhibit a similar trend with respect to VDW isothermal curves on the $(\mathrm{OVp})$ plane, with two main differences. First, no critical point occurs for sufficiently mild density profiles, where all macroisothermal curves are characterized by two extremum points, one maximum and one minimum. Second, a critical macroisothermal curve appears for sufficiently steep density profiles, above (instead of below) which macroisothermal curves exhibit extremum points. For further details refer to the parent paper (C10) and an earlier attempt (Caimmi and Valentinuzzi 2008).

The last inconvenient may be avoided turning Eq. (34) into the following:

$$
\begin{aligned}
& Y_{p} Y_{V} F_{Y}\left(Y_{p}, Y_{V}\right)=Y_{T} ; \\
& Y_{p}=\frac{1}{X_{p}} ; \quad Y_{V}=\frac{1}{X_{V}} ; \quad Y_{T}=\frac{1}{X_{T}} ; \\
& F_{Y}\left(Y_{p}, Y_{V}\right)=F_{X}\left(X_{p}, X_{V}\right) ;
\end{aligned}
$$

as suggested in the parent paper $(\mathrm{C} 10)$.

The existence of a phase transition moving along a selected macroisothermal curve, where the path is a horisontal line ("real" macroisothermal curve) instead of a curve including the extremum points ("actual" macroisothermal curve), must necessarily be assumed as a working hypothesis, due to the analogy between VDW isothermal curves and macroisothermal curves. Unlike the VDW equation of state, Eq. (3), the theoretical macrogas equation of state, Eq. (35a), is not analytically integrable, which implies the procedure used for determining a selected macroisothermal curve, must be numerically performed.

The main steps are (i) calculate the intersections, $Y_{V_{\mathrm{A}}}, Y_{V_{\mathrm{C}}}, Y_{V_{\mathrm{F}}}, Y_{V_{\mathrm{A}}}<$ $Y_{V_{\mathrm{C}}}<Y_{V_{\mathrm{E}}}$, between the generic horizontal line in the $\left(\mathrm{O} Y_{V} Y_{p}\right)$ plane, $Y_{p}=$ const, and the theoretical macrogas equation of state, within the range, $Y_{p_{\mathrm{B}}}<Y_{p}<$ $Y_{p_{\mathrm{D}}}$, where $\mathrm{B}$ and $\mathrm{D}$ denote the extremum points of minimum and maximum, respectively; (ii) calculate the area of the regions, $\mathrm{ABC}$ and $\mathrm{CDE}$; (iii) find the special value, $Y_{p}=Y_{p_{C}}$, which makes the two areas equal; (iv) trace the real macroisothermal curve as a horisontal line connecting the points, $\left(Y_{V_{\mathrm{A}}}, Y_{p_{\mathrm{A}}}\right)$, $\left(Y_{V_{\mathrm{C}}}, Y_{p_{\mathrm{C}}}\right),\left(Y_{V_{\mathrm{E}}}, Y_{p_{\mathrm{E}}}\right), Y_{p_{\mathrm{A}}}=Y_{p_{\mathrm{C}}}=Y_{p_{\mathrm{E}}}=Y_{p_{c}}$. For further details refer to an earlier attempt (C10).

The procedure related to point (ii) above is rather cumbersome and should be performed again with the new variables, $Y_{\mathrm{V}}, Y_{\mathrm{p}}$, and $Y_{\mathrm{T}}$, with respect to an earlier attempt $(\mathrm{C} 10)$. For this reason, the current paper shall be restricted to theoretical macroisothermal curves and related extremum points. In order to preserve the analogy with ideal and VDW gases, the tidal potential energy shall be excluded and included, respectively, in the formulation of the virial 
theorem and related equation of state. The following cases shall be dealt with: UU macrogases, where no critical point occurs; HH macrogases, where the critical point occurs; HN/NH macrogases, where the critical point occurs.

In presence of the critical point, Eq. (35) may be translated into reduced variables, as:

$$
\begin{aligned}
& Y_{p} Y_{V} F_{Y}\left(Y_{p}, Y_{V}\right) \frac{Y_{p_{c}} Y_{V_{c}}}{Y_{T_{c}}}=Y_{T} ; \\
& Y_{p}=\frac{Y_{p}}{Y_{p_{c}}} ; \quad Y Y_{V}=\frac{Y_{V}}{Y_{V_{c}}} ; \quad Y_{T}=\frac{Y_{T}}{Y_{T_{c}}} ; \\
& F_{Y}\left(Y_{p}, Y_{V}\right)=F_{Y}\left(Y_{p} Y_{p_{c}}, Y_{V} Y_{V_{c}}\right) ;
\end{aligned}
$$

where $Y_{p_{c}}, Y_{V_{c}}, Y_{T_{c}}$, are the values of the variables related to the critical point. The counterpart of Eq. (36a) for ideal macrogases reads:

$$
Y_{p} Y_{V} G_{Y}\left(Y_{p}, Y_{V}\right) \frac{Y_{p_{c}} Y_{V_{c}}}{Y_{T_{c}}}=Y_{T}
$$

where $G_{Y}\left(Y_{p}, Y_{V}\right)$ is the expression of $F_{Y}\left(Y_{p}, Y_{V}\right)$ where the interaction terms are omitted. For further details refer to an earlier attempt $(\mathrm{C} 10)$. Accordingly, the equation of state for ideal macrogases where $G_{Y}\left(Y_{p}, Y_{V}\right) Y_{p_{c}} /$ $\left(Y_{V_{c}} Y_{T_{c}}\right)=3 / 8$, coincides with its counterpart related to ideal gases, conformly to Eq. (11).

Macroisothermal curves related to IUU (tidal potential energy excluded) and AUU (tidal potential energy included) macrogases, are plotted in Fig. 4 , left and right panel, respectively, for values of the macrotemperature, $Y_{\mathrm{T}}=$ 20/23, 20/22, 20/21, 20/20, 20/19, 20/18, from bottom to top. The coordinates, $Y_{\mathrm{V}}, Y_{\mathrm{p}}, Y_{\mathrm{T}}$, may be conceived as normalized to their fictitious critical counterparts, $Y_{V_{\mathrm{c}}}=1, Y_{p_{\mathrm{c}}}=1, Y_{T_{\mathrm{c}}}=1(\mathrm{C} 10)$. The comparison with ideal and VDW gases, plotted in Fig. 1, shows a similar trend, except the absence of a critical macroisothermal curve, above which the extremum points disappear.

Macroisothermal curves related to IHH (tidal potential energy excluded) and AHH (tidal potential energy included) macrogases, are plotted in Fig. 5, left and right panels, respectively, for infinitely extended subsystems and values of the reduced macrotemperature, $Y_{\mathrm{T}}=Y_{\mathrm{T}} / Y_{T_{\mathrm{C}}}=20 / 23,20 / 22,20 / 21$, 20/20, 20/19, 20/18, from bottom to top. The general case of bounded subsystems makes only little changes. The comparison with ideal and VDW gases, plotted in Fig. 1, shows a similar trend where macroisothermal curves are more extended along the horisontal direction with respect to isothermal curves. 
Macroisothermal curves related to IHN/NH (tidal potential energy excluded) and AHN/NH (tidal potential energy included) macrogases, are plotted in Fig. 6, left and right panels, respectively, for infinitely extended subsystems and values of the reduced macrotemperature, $\mathrm{Y}_{\mathrm{T}}=Y_{\mathrm{T}} / Y_{T_{\mathrm{c}}}=20 / 23$, $20 / 22,20 / 21,20 / 20,20 / 19,20 / 18$, from bottom to top. The general case of bounded subsystems makes only little changes for AHN/NH macrogases, while the scale change tends to disappear for IHN/NH macrogases. The comparison with ideal and VDW gases, plotted in Fig. 1, shows a similar trend where macroisothermal curves are more extended along the horisontal direction with respect to isothermal curves, and the occurrence of a scale difference for ideal macrogases. The last is due to a mass divergence for infinitely extended $\mathrm{N}$ density profiles, which makes tidal effects higly increase.

The comparison between the VDW critical isothermal curve and its counterparts related to $\mathrm{HH}$ and $\mathrm{HN} / \mathrm{NH}$ macrogases is shown in Fig. 7. The broken curve is the same as in Fig. 2. Accordingly, the vapour and the liquid phase of ordinary fluids coexist within the bell-shaped region bounded by the broken curve. Both $\mathrm{HH}$ and $\mathrm{HN} / \mathrm{NH}$ macroisothermal curves are more extended along the horisontal direction with respect to VDW isothermal curves, which implies a more flattened counterpart of the above mentioned bell-shaped region. The critical point belongs to all curves.

\section{Discussion and conclusion}

Tidal interactions between neighbourhing bodies span across the whole admissible range of lengths in nature: from, say, atoms and molecules to galaxies and clusters of galaxies i.e. from micro to macrocosmos. Ordinary fluids are collisional, which makes the stress tensor be isotropic and the velocity distribution obey the Maxwell's law. Tidal interactions (electromagnetic in nature) therein act between colliding particles (e.g., LL67, Chap. VII, §74). Astrophysical fluids are collisionless, which makes the stress tensor be anisotropic and the velocity distribution no longer obey the Maxwell's law. Tidal interactions (gravitational in nature) therein act between a single particle and the system as a whole (e.g., C10).

In both cases, an equation of state can be formulated in reduced variables: the VDW equation for ordinary fluids and an equation which depends on the density profiles for astrophysical fluids. For sufficiently mild density profiles, macroisothermal curves are characterized by the occurrence of two extremum points, similarly to isothermal curves where a transition from liquid to gaseous phase takes place, or vice versa. For sufficiently steep density profiles, a critical macroisothermal curve exhibits a single horisontal inflex- 
ion point, which defines the critical point. Macroisothermal curves below and above the critical one, show two or no extremum point, respectively, in complete analogy with VDW isothermal curves. In any case, the existence of an equation of state in reduced variables implies the validity of the law of corresponding states for macrogases with assigned density profiles.

For astrophysical fluids, the existence of a phase transition must necessarily be assumed as a working hypothesis by analogy with ordinary fluids. The phase transition has to be conceived between gas and stars, and the $\left(\mathrm{OY} / V Y_{p}\right)$ plane may be divided into three parts, namely (i) a region bounded by the critical macroisothermal curve on the left of the critical point, and the locus of onset of phase transition on the right of the critical point, where only gas exists; (ii) a region bounded by the critical macroisothermal curve on the left of the critical point, the locus of onset of phase transition on the left of the critical point, and the vertical axis, where only stars exist; (iii) a region bounded by the locus of onset of phase transition, and the horisontal axis, where gas and stars coexist. The locus of onset of phase transition, not shown in Fig. 7 for reasons explained above, is similar to its counterpart related to ordinary fluids, represented by the bell-shaped curve in Fig. 7, but more extended along the horisontal direction.

In this view, elliptical and S0 galaxies lie on (ii) region unless hosting hot interstellar gas, and the same holds for globular clusters; spiral, irregular, and dwarf spheroidal galaxies lie on (iii) region, and the same holds for cluster of galaxies; gas clouds in absence of star formation lie on (i) region, and the same holds for hypothetic galaxies with no stars.

In conclusion, van der Waals' two great discoveries, more specifically a gas equation of state where tidal interactions between molecules are taken into account, and the law of corresponding states, related to microcosmos, find a counterpart with regard to macrocosmos. After a century since the awarding of the Nobel Prize in Physics, van der Waals' ideas are still valid and helpful to day for a full understanding of the universe.

\section{References}

[1] Caimmi, R.: Serbian Astron. J. 180, 19 (2010) (C10)

[2] Caimmi, R., Valentinuzzi, T.: Serbian Astron. J. 177, 15 (2008)

[3] Chandrasekhar, S., An Introduction to the Study of the Stellar Structure, University of Chicago Press (1939)

[4] Hernquist, L.: Astrophys. J. 356, 359 (1990) 
[5] Landau, L., Lifchitz, E.: Physique Statistique, Mir, Moscow (1967) (LL67)

[6] Navarro, J.F., Frenk, C.S., White, S.D.M.: Mon. Not. R. Astron. Soc. $275,720(1995)$

[7] Navarro, J.F., Frenk, C.S., White, S.D.M.: Astrophys. J. 462, 563 (1996)

[8] Navarro, J.F., Frenk, C.S., White, S.D.M.: Astrophys. J. 490, 493 (1997)

[9] Nobel Lectures, Physics 1901-1921: Elsevier Publishing Company, Amsterdam (1967)

[10] Rostagni, A.: Meccanica e Termodinamica, ed. Libreria Universitaria di G. Randi, Padova (1957)

[11] Spiegel, M.R.: Mathematical Handbook, Schaum's Outline Series, McGraw-Hill, Inc., New York (1968)

[12] van der Waals, J.D.: Over de Continuited van den Gas-en Vloeistoftoestand (Doctoral Thesis) (1873) 


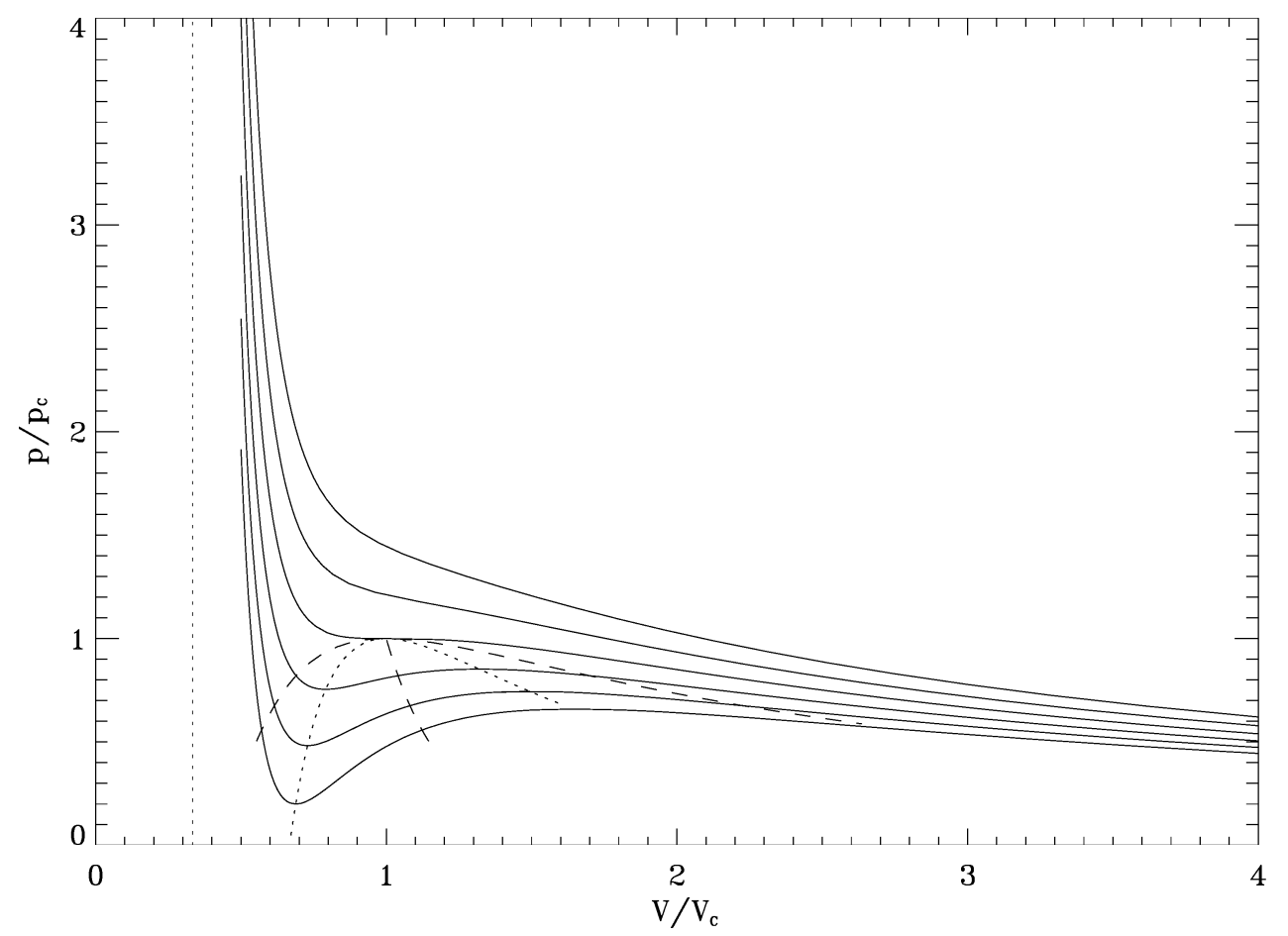

Figure 2: Same as in Fig. 1 (right panel), where the occurrence (within the bell-shaped area bounded by the dashed curve) of saturated vapour is considered. Above the critical isothermal curve $\left(T=T_{\mathrm{c}}\right)$ the trend is similar with respect to ideal gases. Below the critical isothermal curve and on the right of the dashed curve, the gas still behaves as an ideal gas. Below the critical isothermal curve and on the left of the dashed curve, the liquid shows little change in volume as the pressure rises. Within the bell-shaped area bounded by the dashed curve, the liquid phase is in equilibrium with the saturated vapour phase. A diminished volume implies smaller saturated vapour fraction and larger liquid fraction at constant pressure, and vice versa. The VDW equation of state is no longer valid in this region. The dashed curve (including the central branch) is the locus of intersection between VDW and real isothermal curves, the latter being related to constant pressure where liquid and vapour phases coexist. The dotted curve is the locus of VDW isothermal extremum points. 


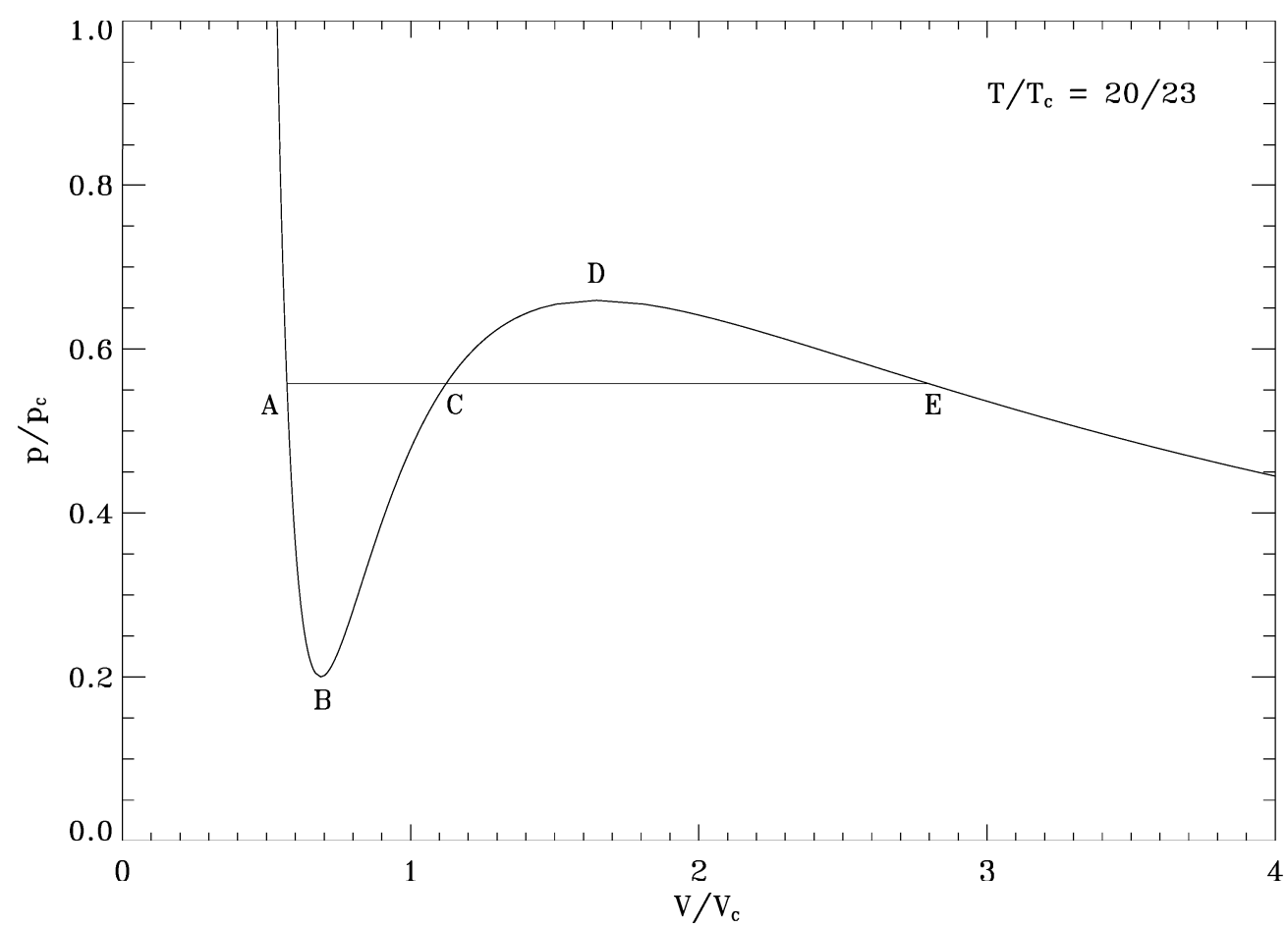

Figure 3: A specific $\left(T / T_{\mathrm{c}}=20 / 23\right) \mathrm{VDW}$ and corresponding real isothermal curve. The above mentioned curves coincide within the range, $V \leq V_{\mathrm{A}}$ and $V \geq V_{\mathrm{E}}$. The VDW isothermal curve exhibits two extremum points: a minimum, $\mathrm{B}$, and a maximum, $\mathrm{D}$, while the real isothermal curve is flat within the range, $V_{\mathrm{A}} \leq V \leq V_{\mathrm{E}}$. Configurations related to the VDW isothermal curve within the range, $V_{\mathrm{A}} \leq V \leq V_{\mathrm{B}}$ (due to tension forces acting on the particles yielding superheated liquid), and $V_{\mathrm{D}} \leq V \leq V_{\mathrm{E}}$ (due to the occurrence of undercooled vapour), may be obtained under special conditions, while configurations within the range, $V_{\mathrm{B}} \leq V \leq V_{\mathrm{D}}$, are always unstable. The volumes, $V_{\mathrm{A}}$ and $V_{\mathrm{E}}$, correspond to the maximum value in presence of the sole liquid phase and the minimum value in presence of the sole vapour phase, respectively. The regions, $\mathrm{ABC}$ and $\mathrm{CDE}$, have equal area. For further details refer to the text. 

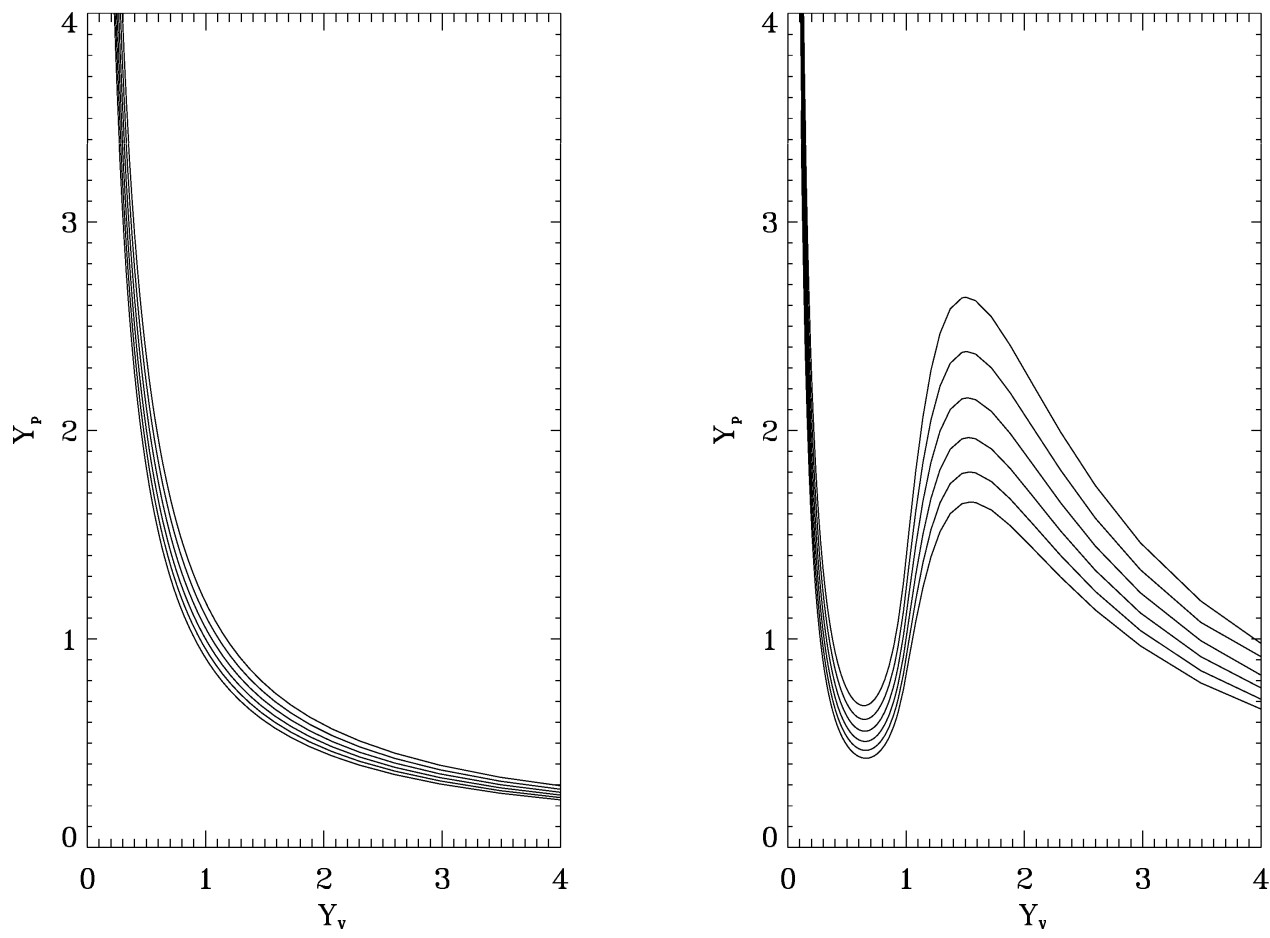

Figure 4: Macroisothermal curves related to IUU (left panel) and AUU (right panel) macrogases, respectively. Macroisothermal curves (from bottom to top) correspond to $Y_{\mathrm{T}}=20 / 23,20 / 22,20 / 21,20 / 20,20 / 19,20 / 18$. No critical macroisothermal curve exists, above which the extremum points disappear. The coordinates, $Y_{\mathrm{V}}, Y_{\mathrm{p}}, Y_{\mathrm{T}}$, may be conceived as normalized to their fictitious critical counterparts, $Y_{V_{\mathrm{c}}}=1, Y_{p_{\mathrm{c}}}=1, Y_{T_{\mathrm{c}}}=1$. 

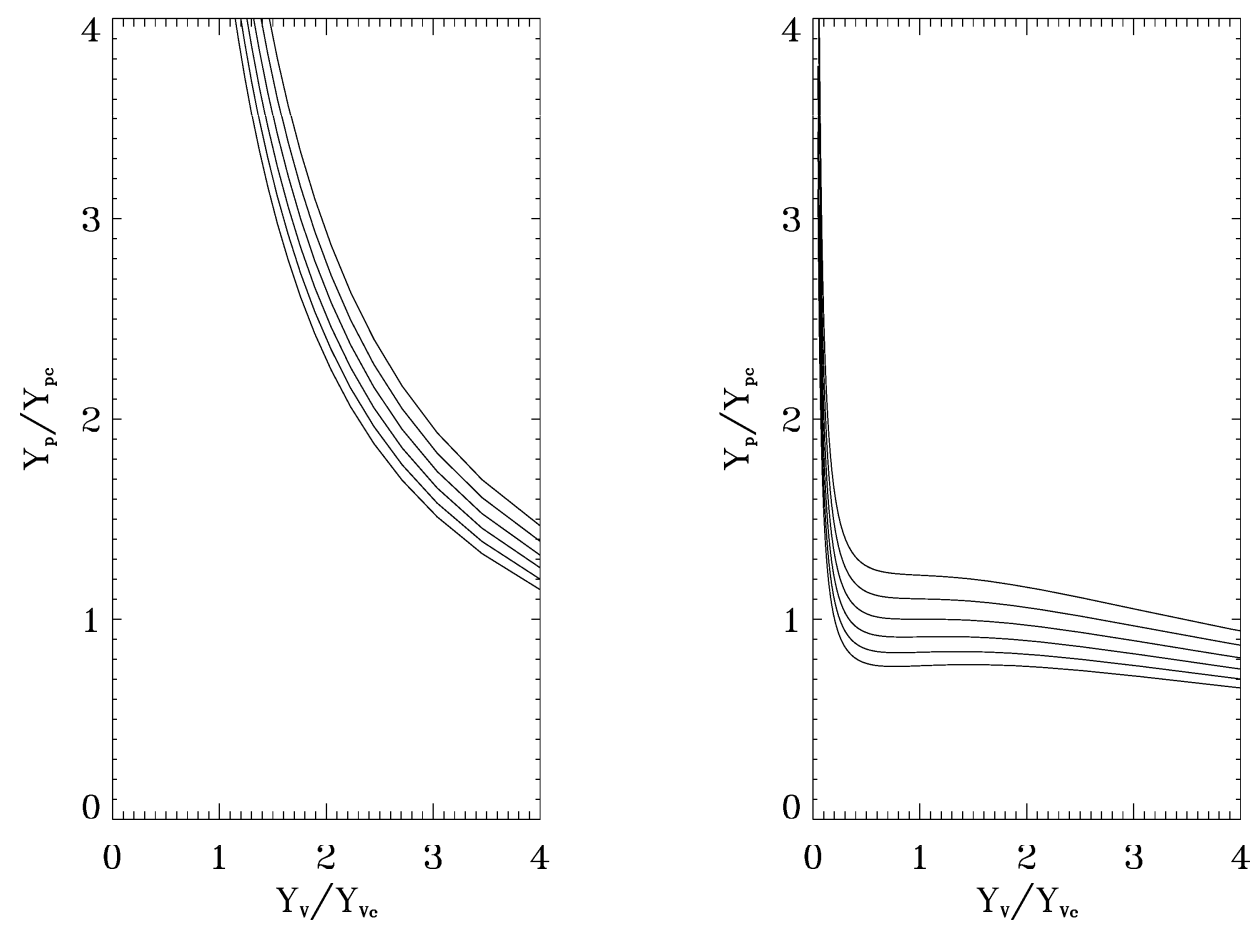

Figure 5: Macroisothermal curves $\left(Y_{\mathrm{p}}=Y_{\mathrm{p}} / Y_{p_{c}}\right.$ vs. $\left.Y_{\mathrm{V}}=Y_{\mathrm{V}} / Y_{V_{c}}\right)$ related to IHH (left panels) and AHH (right panels) macrogases, respectively, for infinitely extended subsystems. Macroisothermal curves (from bottom to top) correspond to $Y_{\mathrm{T}}=Y_{\mathrm{T}} / Y_{T_{\mathrm{c}}}=20 / 23,20 / 22,20 / 21,20 / 20,20 / 19,20 / 18$. The general case of bounded subsystems makes only little changes. 

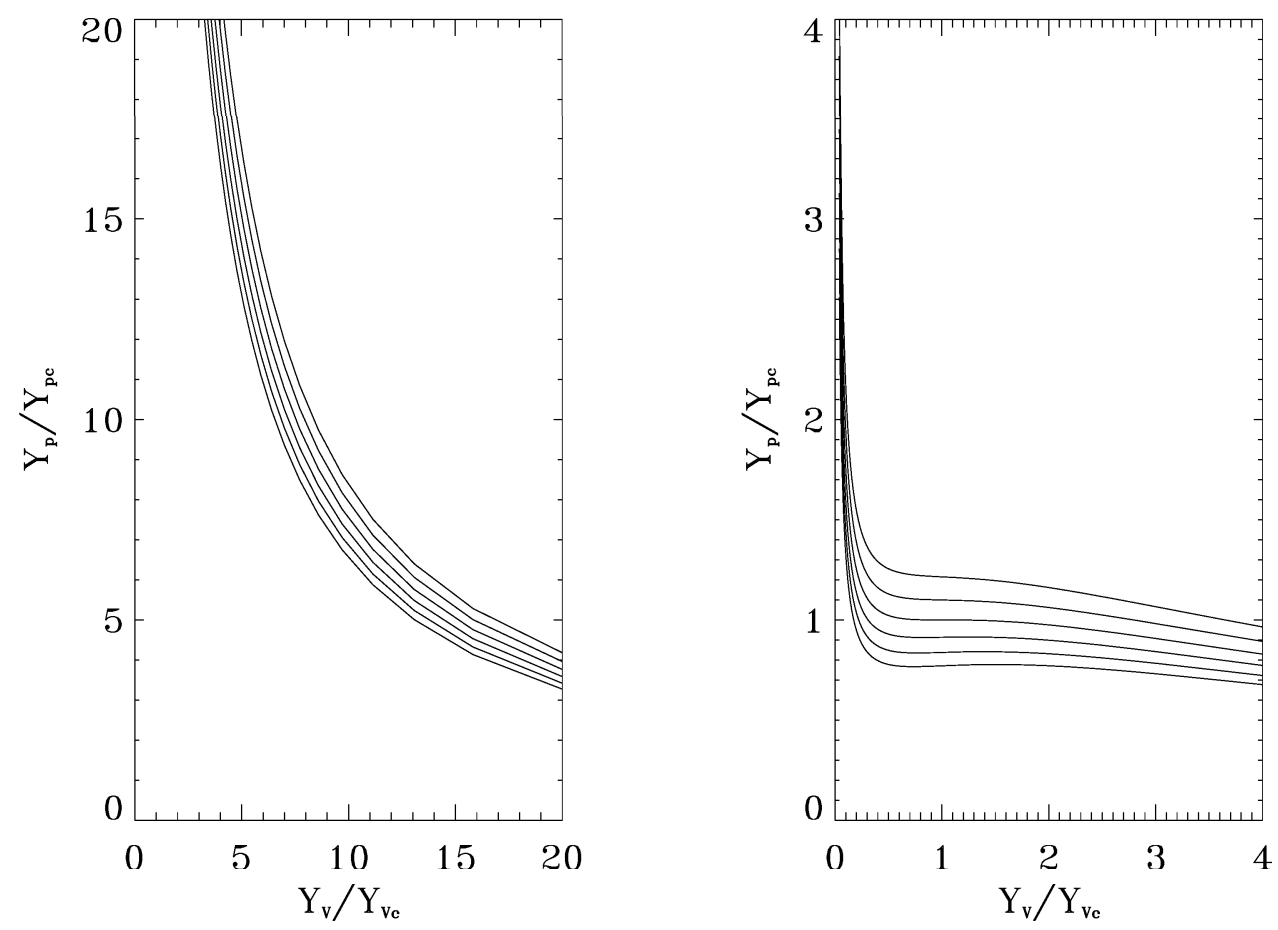

Figure 6: Macroisothermal curves $\left(\mathrm{Y}_{\mathrm{p}}=Y_{\mathrm{p}} / Y_{p_{c}}\right.$ vs. $\left.\quad \mathrm{Y}_{\mathrm{V}}=Y_{\mathrm{V}} / Y_{V_{c}}\right)$ related to IHN/NH (left panels, to be noted the scale difference) and $\mathrm{AHN} / \mathrm{NH}$ (right panels) macrogases, respectively, for infinitely extended subsystems. Macroisothermal curves (from bottom to top) correspond to $Y_{\mathrm{T}}=Y_{\mathrm{T}} / Y_{T_{\mathrm{c}}}=23 / 20,22 / 20,21 / 20,20 / 20,19 / 20,18 / 20$. The general case of bounded subsystems makes only little changes for AHN/NH macrogases, while the scale difference tends to disappear for IHN/NH macrogases. 


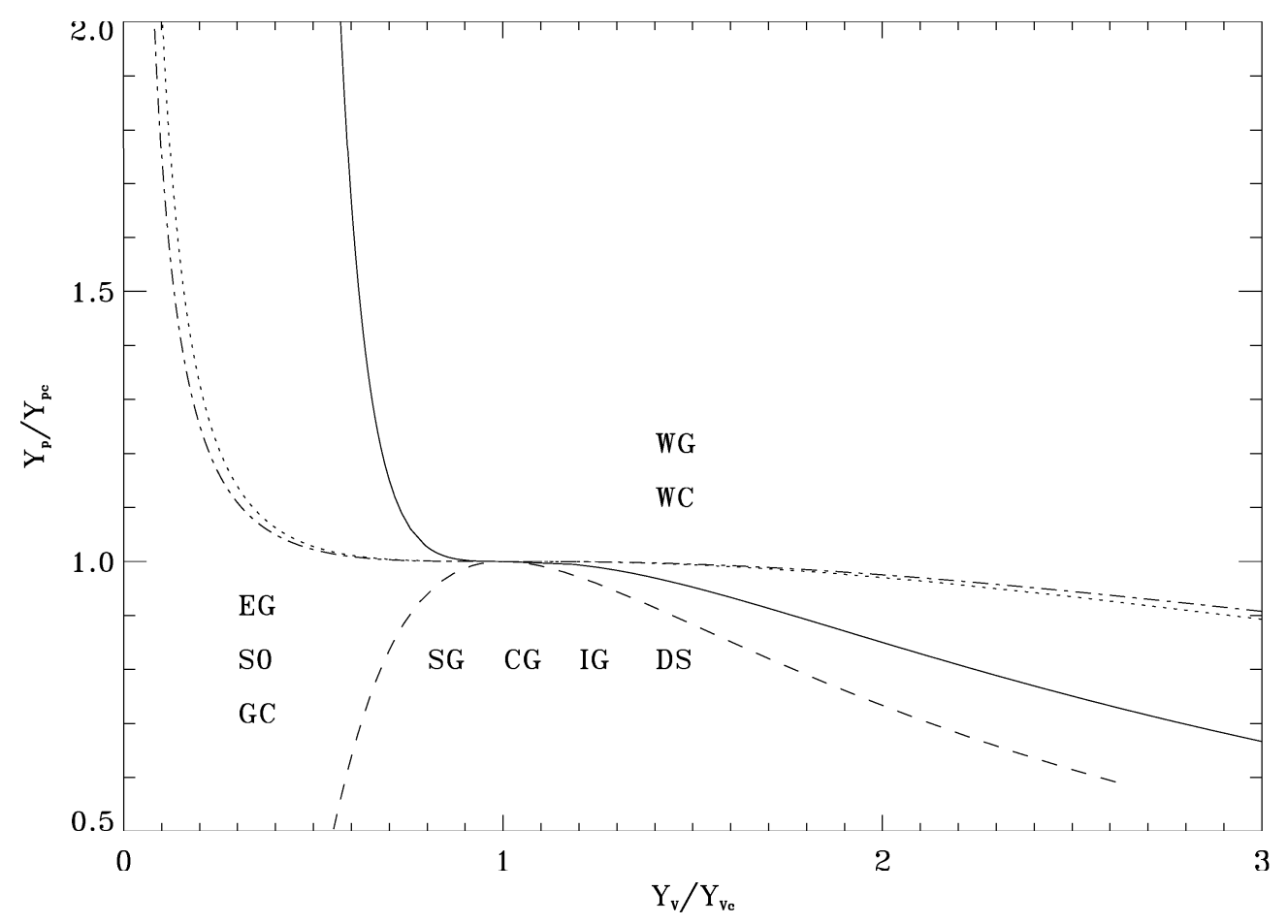

Figure 7: Comparison between VDW critical isothermal curve (full), HH critical macroisothermal curve (dotted) and HN/NH critical macroisothermal curve (dot-dashed). With regard to ordinary fluids, the vapour and the liquid phase coexist within the bell-shaped region bounded by the dashed curve and, in addition, $Y_{\mathrm{V}}=V, Y_{\mathrm{p}}=p$. More extended (along the horisontal direction) bell-shaped regions are expected for $\mathrm{HH}$ and $\mathrm{HN} / \mathrm{NH}$ macroisothermal curves. The critical point belongs to all curves. Different letters denote the expected location of different astrophysical systems. Caption: EG - elliptical galaxies; S0 - lenticular galaxies; SG - spiral galaxies including barred; IG - irregular galaxies; DS - dwarf spheroidal galaxies; GC - globular clusters; CG - clusters of galaxies; WC - wholly gaseous clouds i.e. in absence of star formation; WG - (hypothetical) wholly gaseous galaxies i.e. in absence of star formation. 


\title{
WEIGHT OF THE THERMO-INSULATED CONTAINER EQUIPPED WITH ELECTRIC HEATER
}

\author{
Alexander L. Dmitriev \\ St. Petersburg National Research University of Information Technologies, Mechanics and Optics,
}

St. Petersburg, 49 Kronverksky Prospect, 197101, Russia

Experiment with weighing of the thermo-insulated container in form of three tight metal vessels, in which the internal one is heated up by an electric spiral, is briefly described. Results of the experiment show a rather strong temperature reduction of weight of the vessel made from titan with a relative value in the order of $10^{-6} \mathrm{~K}^{-1}$.

Keywords: gravitation, weight, temperature

Researches of influence of temperature of bodies on results of their exact weighing are actual not only in gravimetry and metrology of mass, but also in connection with importance of this problem for physics of gravitation. The body temperature is a measure of energy of chaotic movement of the microparticles it is made of, which is directly connected with their speed and acceleration; the temperature - the factor describing the electromagnetic interaction of particles of a body. Forces of gravitation also cause the acceleration of bodies, and researches of deep and not trivial interrelation of acceleration and gravitation have fundamental importance. ${ }^{1-4}$ Last years these researches draw special attention in connection with the fact that in a number of experiments there is observed a rather strong negative dependence of the force of gravitation on the temperature. ${ }^{5-7}$

A simple experiment with weighing of the tight thermo-insulated container, inside which the electric heater is placed, has been described below.

The design of container in form of three thoroughly hermetically sealed rigid metal vessels, enclosed in each other, is shown in FIG. 1.

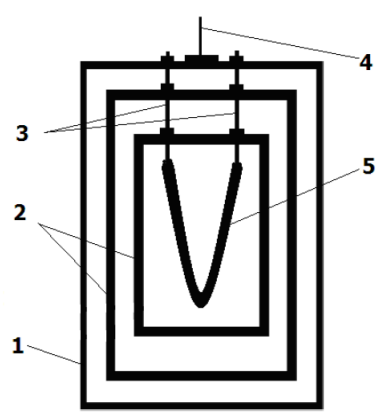

FIG. 1. The design of the container. 1 - a vessel made of steel, 2 - vessels made of titan, 3 - copper electrodes, 4 - a suspension bracket, 5 - an electric spiral.

Full weight of the container is $166.8 \mathrm{~g}$, diameter of the external steel cylinder is $40 \mathrm{~mm}$, height is $53 \mathrm{~mm}$, thickness of walls of the steel cylinder is $1.5 \mathrm{~mm}$, walls of the titan cylinders is $2 \mathrm{~mm}$. Heater - a wire spiral made of Nichrome with weight of $540 \mathrm{mg}$ and electrical resistance of $10.5 \Omega$; the voltage of the power source is $12.5 \mathrm{~V}$, duration of the spiral heating is $20 \mathrm{~s}$.

Weighing of the container before and after the heater switching ON was made on analytical balance of ADV200 make by the elongation method with the period of readout of the current values of weight being about $18 \mathrm{~s}$. Duration of operations of removal and installation of the container for connection to the power source is $1.5 \mathrm{~min}$, accuracy of selective readouts of the container weight is not worse than $50 \mathrm{mcg}$. An example of typical experimental time dependence of the container weight is given in FIG. 2. 


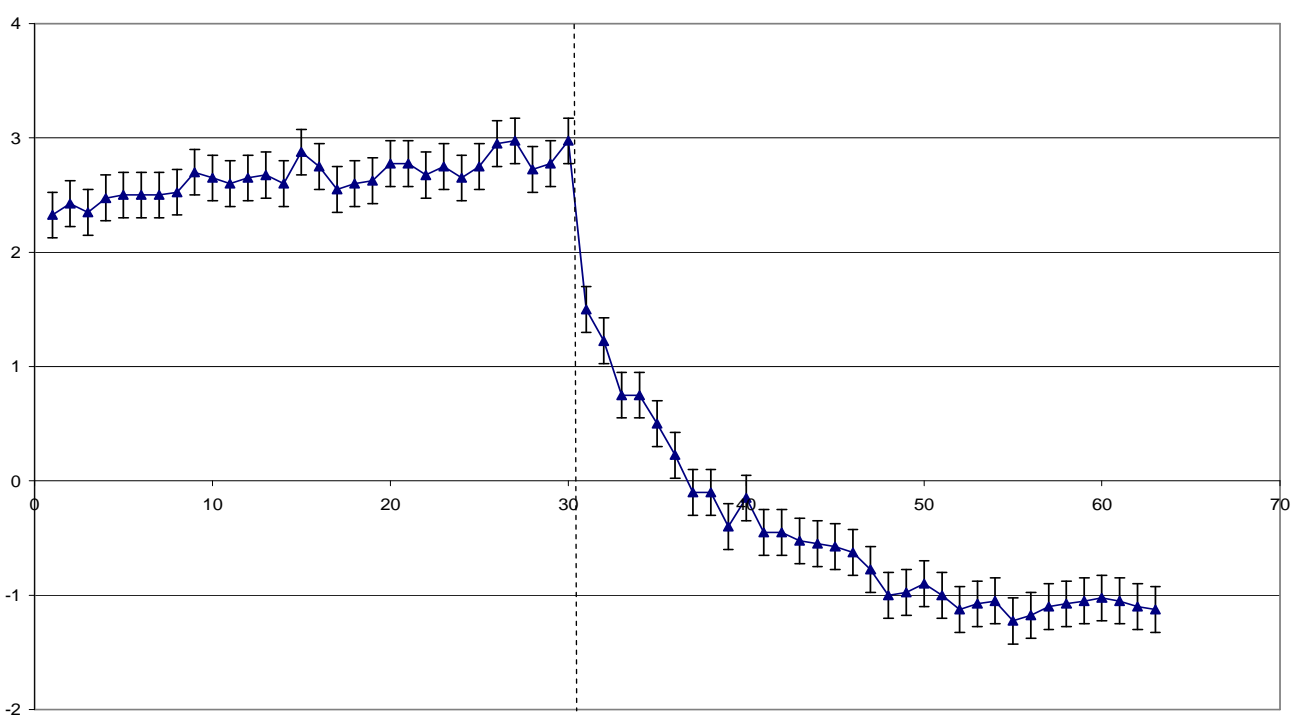

FIG. 2. Time dependence of change of the container apparent weight. The dash line specifies the "moment" of ON and OFF of the heater. One division on the vertical scale corresponds to $100 \mathrm{mcg}$, the period of weight readouts is $18.2 \mathrm{~S}$.

Significant, more than $300 \mathrm{mcg}$ in value, reduction of the container weight during heating and first 2-3 minutes after action of the heater, has regular character and is not connected to errors of operation of removal and installation of the container on the beam of analytical balance (it is proved to be true by the repeated careful measurements).

Principal causes of changes in weight of the hermetically sealed container with the heated up spiral are:

- change of buoyancy of a weighed body, owing to temperature-dependent change of volume of the external steel vessel;

- convective flows of air near the wall of the external vessel, owing to its being heated;

- temperature changes of weight of the electrical spiral and weights of the container, to the greatest degree, weights of the internal cylinder.

Change of the surface temperature of the external cylinder is shown in FIG. 3.

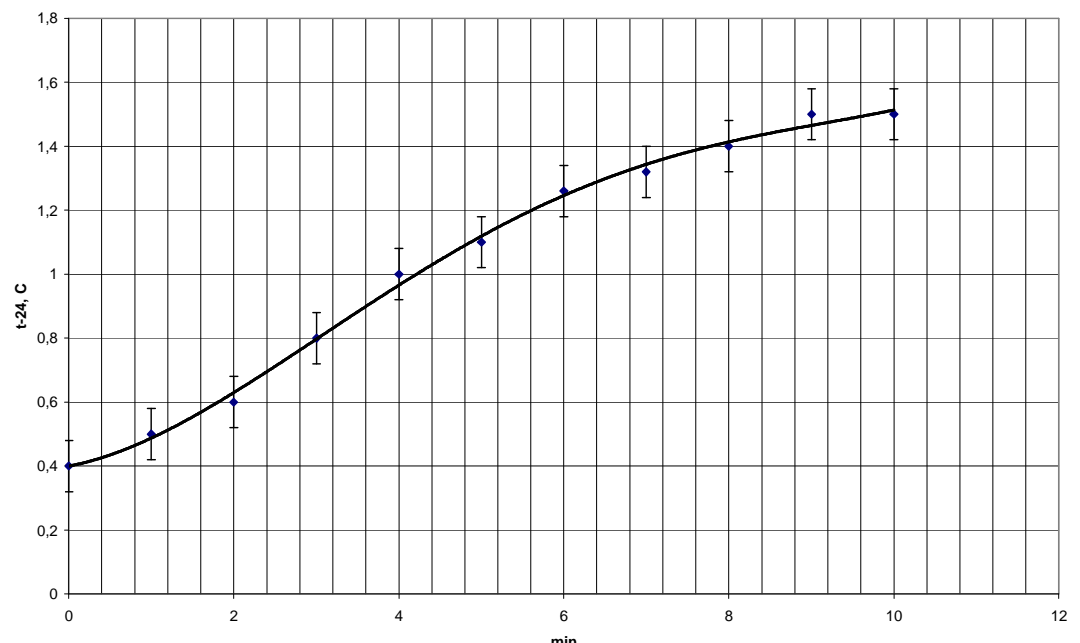

FIG. 3. Change of the surface temperature $t$ of the external cylinder. Time of heating is $20 \mathrm{~s}$, the moment "0" corresponds to ON position of the heater.

Obviously, in the first three minutes of measurements the increase $\Delta T$ of temperature of the external steel cylinder does not exceed $0.4 K$.

Change $\Delta m_{1}$ of apparent weight of the container, caused by change of volume of the steel cylinder owing to 
thermal expansion of its material is equal to:

$$
\Delta m_{1}=\frac{3 \pi}{4} \rho d^{2} h \alpha \Delta T
$$

where $\rho$ - density of air, $\alpha$ - factor of linear expansion of material of the cylinder, $d$ - its diameter, $h$ - height. At $\rho=1.19 \mathrm{~kg} / \mathrm{m}^{3}, \alpha=11.9 \cdot 10^{-6}$ and $\Delta T=0.4 K \Delta m_{1} \approx 1 \mathrm{mcg}$.

Change $\Delta m_{2}$ of apparent weight of the container, caused by deformations of walls of the cylindrical vessel, owing to temperature change of air pressure $\Delta P$ within its volume, as it is possible to show on the basis of the theory of elasticity ${ }^{8}$, is equal to:

$$
\Delta m_{2}=\frac{\pi \rho h d^{3} \Delta P}{4 \delta E}+\frac{\pi \rho d^{3}}{16} \sqrt[3]{\frac{3(1-v) d \Delta P}{2 \delta E}},
$$

where $\delta$-thickness of walls, $E$ - the modulus of elasticity and $v$ - Poisson's ratio. The size $\Delta P$ is connected to change of temperature $\Delta \bar{T}$ of air within the volume of the external cylinder $\Delta P=P \Delta \bar{T} / T$, where $P$ - normal pressure of atmosphere and $T$ - temperature of air in the cylinder. For example, at $\Delta \bar{T}=1 K, T \approx 298 K$, $P \approx 1.013 \cdot 10^{5} \mathrm{~N} / \mathrm{m}^{2}, \Delta P \approx 340 \mathrm{~N} / \mathrm{m}^{2}$. At $\delta=1.5 \mathrm{~mm}, E \approx 2 \cdot 10^{11} \mathrm{~N} / \mathrm{m}^{2}, v \approx 0.3, \Delta m_{2} \approx 50 \mathrm{mcg}$.

The given estimate is overestimated, as in the second addend of formula 2, describing deformation of face walls of a vessel, such walls are presented by thin membranes; actually, the deflection of end faces is less than it is supposed in conclusion 2.

The change $\Delta m_{3}$ of apparent weight of the container, caused by air convection due to difference $\Delta T$ of temperatures of surface of the external steel cylinder and temperatures of air in the closed box of analytical balance, will be estimated on the basis of ${ }^{9}$ according to which

$$
\frac{\Delta m_{3}}{A d^{1 / 4} \Delta T^{3 / 4}}=9.2 \cdot 10^{-7} \mathrm{gcm}^{-9 / 4} K^{-3 / 4}
$$

where the area of lateral surface of the cylinder is $A=\pi d h$. With the maximum difference $\Delta T=0.4 K$, $\Delta m_{3} \approx 40 \mathrm{mcg}$.

Reduction of weight of the container during the first 2-3 minutes of measurements is equal to about $350 \mathrm{mcg}$ which exceeds the sum $\left(\Delta m_{1}+\Delta m_{2}+\Delta m_{3}\right)$ by more than three times.

The observed "defect" of masse $\Delta m_{4}$ with value of $260 \mathrm{mcg}$ can be explained by temperature change of weight of the container with weights $m_{v}$ of the internal titan vessel in the greatest degree.

As shown by the thermo-physical calculations, the change $\Delta T_{v}$ of temperature of the internal vessel in the first minutes from the moment $\mathrm{ON}$ of short-time switching on of the heater is equal to about $10 \mathrm{~K}\left(\Delta T_{v}=\Delta Q / m_{v} c\right.$, where $\Delta Q \approx 100 \mathrm{~J}$ - the energy transferred to a vessel during heat exchange $m_{v}=0.02 \mathrm{~kg}, c=574 \mathrm{~J} / \mathrm{kg} \cdot \mathrm{K}$ the specific thermal capacity of titan).

The factor $\gamma$ describing relative temperature change of weight of an internal vessel,

$$
\gamma=\frac{\Delta m_{4}}{m_{v} \Delta T_{v}}
$$

which is not less than $1.5 \cdot 10^{-6} \mathrm{~K}^{-1}$ that is close to the values of this factor earlier obtained for metals. ${ }^{5,6}$

The detailed description of dynamics of temperature change of the container weight can be executed with account 
for specific features of the container design, and also for the design and the mode of operation of the electric heater.

So, the described experiment confirms the rather strong negative temperature dependence of weight of the container, containing a heated up vessel made of titan. Researches of temperature dependence of weight of materials, conducted in a wide range of temperatures, will allow to expand the existing concepts of physics of gravitational interaction and its connection with electromagnetism.

\section{References}

1 A. L. Dmitriev, Russian Physics Journal, 44 (12), 1323-1327 (2001).

${ }^{2}$ A. L. Dmitriev, $18^{\text {th }}$ International Conference on General Relativity and Gravitation (GRG18), Abstract Book, 77 (2007).

${ }^{3}$ A. L. Dmitriev, AIP Conference Proceedings 969, 1163 (2008).

${ }^{4}$ A. L. Dmitriev, AIP Conference Proceedings 1103, 345 (2009).

${ }^{5}$ A. L. Dmitriev, E. M. Nikushchenko, V. S. Snegov, Measurement Techniques, 46 (2), 115 (2003).

${ }^{6}$ Liangzao Fan, Jinsong Feng, Wuqing Liu, Engineering Sciences, China 12 (2), 9 (2010).

${ }^{7}$ A. L. Dmitriev, Engineering Physics, No 3, 48 (2012) (in Russian)

${ }^{8}$ Yu. N. Rabotnov, Soprotivlenie Materialov, GIFML, Moscow (1962) (in Russian)

${ }^{9}$ M. Glaser, Metrologia, 27, No2, 95 (1990) 


\title{
Relativistic Theory of Black Holes
}

\author{
Daniele Sasso *
}

\begin{abstract}
The gravitational theory is the most accredited theory for explaining black holes. In this paper we present a new interpretation based on the relativistic theory that explains black holes as a consequence of the relativistic speed of departure between the speed of celestial system and the speed of both light and quantum rays at very high energy, calculated with respect to the observer.
\end{abstract}

\section{Historical introduction to black holes}

The term "black hole" was introduced by American physicist J.A. Wheeler because everything, inclusing the light, that went into that astronomical zone wasn' $t$ able to get out and consequently it appeared black.

In the $18^{\text {th }}$ century Laplace and Michell hypothesized for the first time the existence of a celestial body provided with a greatest mass that was able to cause an escape velocity greater than the speed of light for which neither light was able to resist the strongest gravitational force generated by the celestial body.

This hypothesis got on with Newton's corpuscular theory of light but not with the wave theory: on this account the concept of black hole was abandoned.

Some month after the publication of General Relativity by Einstein (1916) the black hole was again contemplated because gravitation in GR was considered a geometric variation of the space and not a force. In 1919 Eddington on the occasion of a total solar eclipse ${ }^{[1]}$ measured the deflection of light coming from a remote star when light passed near the sun. He deduced that in place of the sun a greatest celestial mass should have produced a so great deflection of light that this once gone into the even horizon wasn' $t$ able to get out any longer.

More or less in the same years also Karl Schwarzschild calculated that the black hole should have possessed a greatest mass because the calculus implied a smallest radius of the celestial body $\left(R=2 G M / c^{2}\right)$ and consequently in order to have an acceptable value of radius a very great mass was necessary.

It is also suitable to say that lastly a few published papers have denied the existence of black holes ${ }^{[2]}$. Future further experiments will say if black holes represent a physical reality also if already now there are many evidences in this regard. In the gravitational theory the black hole looks like an astronomical monster that devours all what passes in the proximity of its even horizon, the relativistic theory intends also to propose a more friendly explanation of the black hole.

*email:dgsasso@alice.it 


\section{Gravitational theory}

Whether in Newton's or Einstein's gravitational theory black holes can be explained with a very strong intensity of the gravitational field that is caused by a greatest mass. In fact in Newton' $s$ theory the $F_{G}$ gravitational force on a m mass in a point at $r$ distance from the centre of gravity is given by

$$
F_{G}=\frac{G M m}{r^{2}}
$$

where $\mathrm{G}=6,67 \times 10^{-11} \mathrm{Nm}^{2} \mathrm{Kg}^{-2}$ is the constant of universal gravitation and $M$ is the mass of the celestial body generating the gravitational field. The gravitational force is the more strong the more $M$ is great and the more $r$ is small.

A body with $m$ mass in order to leave the surface of $M$ celestial mass has to be provided with an escape force $F_{e}=m a_{e}$ with opposed direction with respect to the direction of the gravitational force. In order to calculate the initial minimum speed $\left(v_{e}\right.$ escape speed) that a $m$ mass has to possess at the $r$ distance for leaving definitively the celestial body it has to be

$$
\frac{G M m}{r^{2}}=-\frac{m d v}{d t}
$$

Being

$$
\frac{\mathrm{d} v}{\mathrm{dt}}=\frac{\mathrm{dv}}{\mathrm{dr}} \frac{\mathrm{dr}}{\mathrm{dt}}
$$

we have

$$
\begin{gathered}
\frac{d v}{d r} \frac{d r}{d t}=-\frac{G M}{r^{2}} \\
v d v=-\frac{G M d r}{r^{2}} \\
v^{2}=\frac{2 G M}{r}
\end{gathered}
$$

and

$$
v_{e}=\sqrt{\frac{2 G M}{r}}
$$

From the (7) equation we deduce that the escape speed depends on both the $r$ distance from the centre of gravity and the $M$ mass of the celestial body. From the (6) equation, in the event of light $(v=c)$, we find again Schwarzschild's radius

$$
R=\frac{2 G M}{c^{2}}
$$


In this theory $\mathrm{R}$ represents the radius of the even horizon because $\mathrm{M}$ mass is concentred into a smallest volume because of the strongest gravitational field. A graphic representation of black hole is given in fig.1.

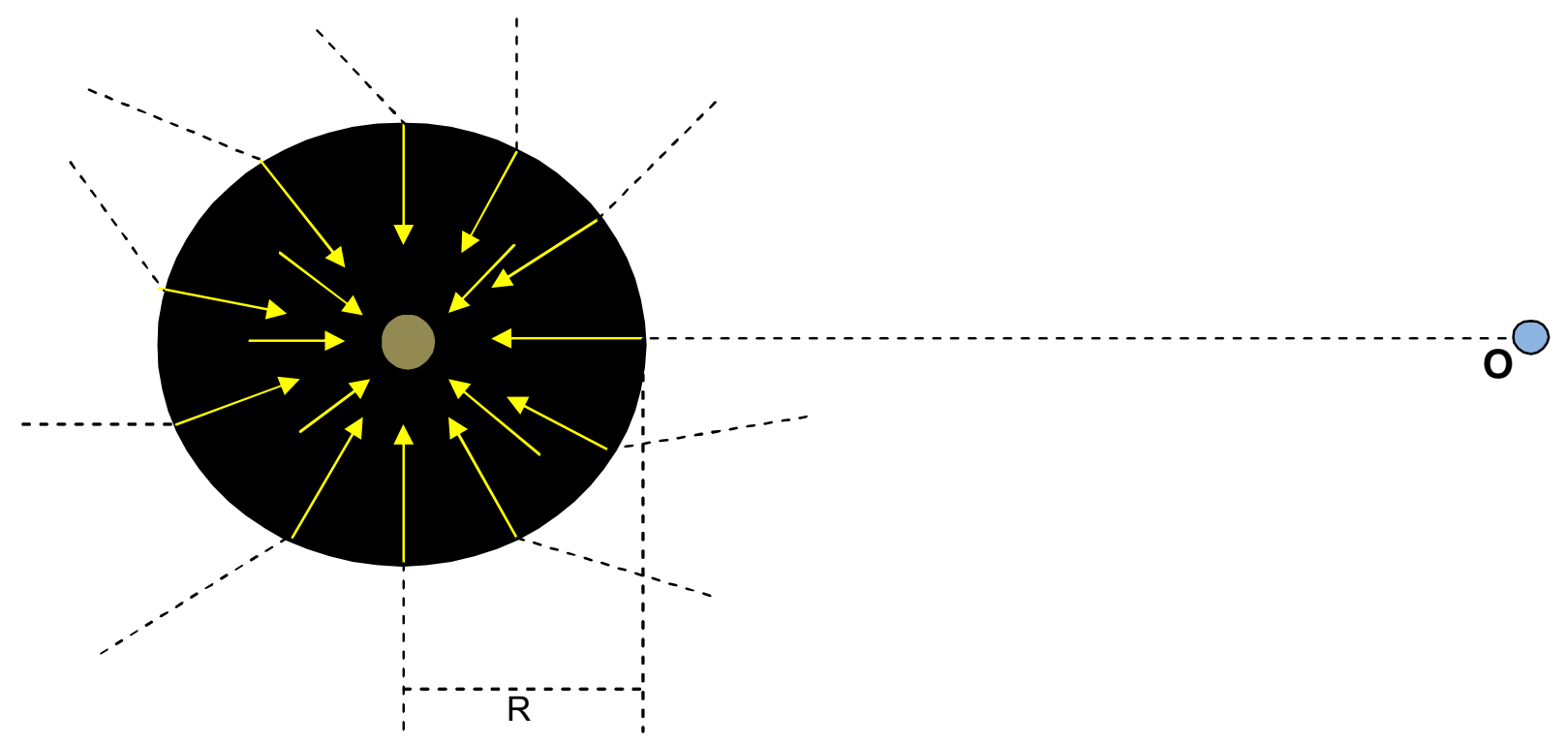

Fig.1 Graphic representation of black hole in the gravitational theory. $\mathrm{R}$ is the radius of the event horizon: all what is inside cannot go out, all what passes very near to the horizon (also the light) is attracted inside. The observer is in the $O$ point.

In the gravitational theory black hole represents an astronomical entity whose prospective motion would have no revealing effect on its behavior. In GR the gravitational force is replaced with the space and time warp that is the more strong the more $\mathrm{M}$ mass is great and consequently the behavior of black hole is equivalent to a suction effect so great that also the light isn't able to go out.

\section{Relativistic theory}

Let us consider an $\mathrm{O}$ observer who is in the origin of the $\mathrm{S}$ reference frame supposed at rest and similarly consider a celestial body which constitutes the S' reference frame. Suppose still that $S^{\prime}$ moves with $V$ velocity in reverse with respect to $S$. Any object launched with speed $u$ from the surface of the celestial body towards the $S$ reference frame along the conjoining line the centre of gravity of $S^{\prime}$ and the $O$ point where the observer is placed (fig.2), has with respect to $S$ the vector velocity ${ }^{[3][4]}$

$$
\mathbf{v}=\mathbf{u}+\mathrm{V}
$$

In concordance with intensities of $\mathbf{V}$ and $\mathbf{u}$ the following cases can happen:

1. $V<u$ : the launched object reaches the $O$ observer with scalar velocity $v=u-V$ in a time $d /(u-V)$ only if the resultant velocity $v$ is greater than the escape speed of the celestial body $v_{e}=\sqrt{2 G M / R}\left(v>v_{e}\right)$. 
2. $V=u$ : the launched object doesn' $t$ reach the $O$ observer and the object staies at a constant distance from $O$.

3. $V>u$ : the launched object doesn't reach the $O$ observer and in any case it moves away from $\mathrm{O}$ with speed $\mathrm{v}=\mathrm{V}-\mathrm{u}$ (this case is represented in fig.2).

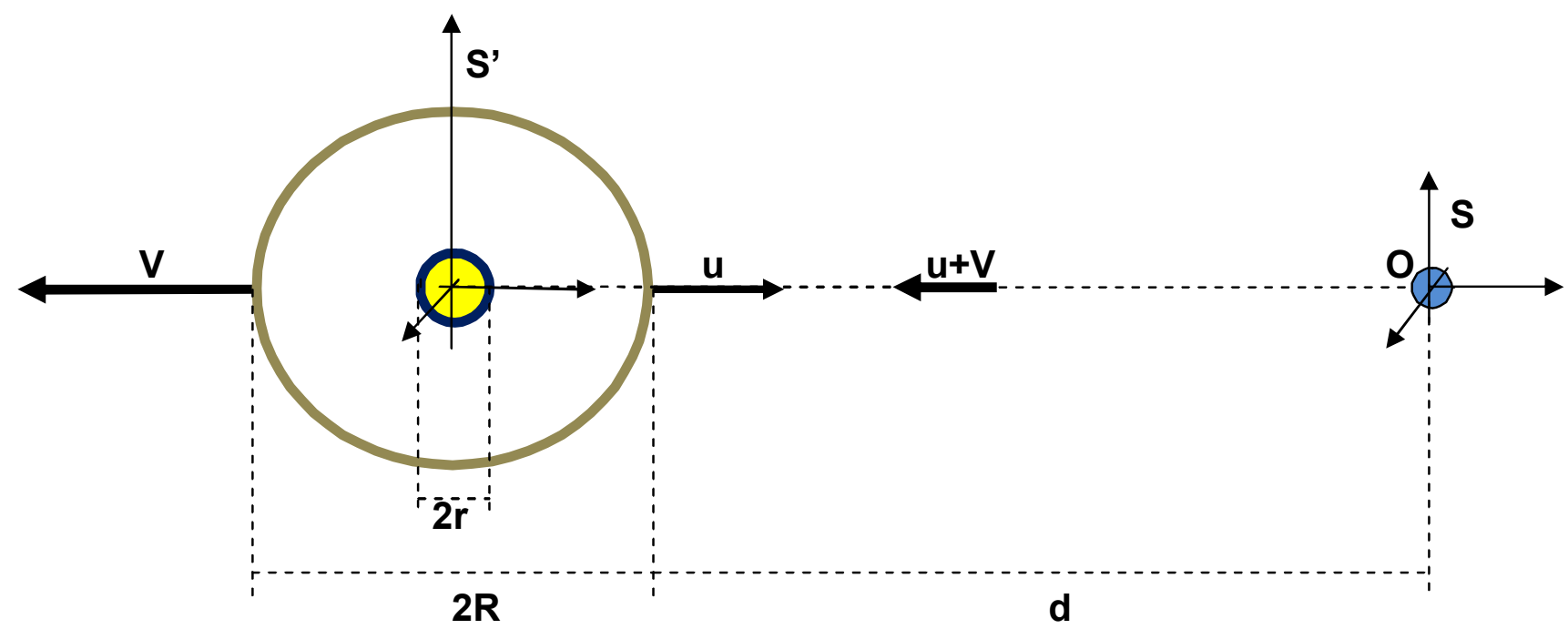

Fig.2 Graphic representation of black hole in the relativistic theory where $\mathbf{V}$ is the speed of departure of black hole with respect to the O observer, $\mathbf{u}$ is the speed of an object launched from the surface of black hole and $\mathbf{u}+\mathbf{V}$ is the speed of the same object with respect to $S$. In figure the case $V>u$ is represented. In the graphic the black hole is represented by a sphere with $R$ radius which because of very great distance from the $O$ observer appears like a small sphere with $r$ radius. $d$ is the distance between the observer and the launch point of the object. In the event of the light the $\mathbf{u}$ speed coincides with $\mathbf{c}$.

In the event of light or energy rays emitted by the celestial body we have $u=c$. The following cases can happen:

1. $V<c$ : the light reaches the $O$ observer with velocity $v=c-V$ in a time $\mathrm{d} /(\mathrm{c}-\mathrm{V})^{[4]}$.

2. $V=c$ : with respect to $O$ observer the light has null velocity and consequently it isn't able to reach the $O$ observer.

3. $V>c$ : the light isn't able to reach the $O$ observer.

In the both cases 2. and 3. the celestial body behaves like a black hole. If during its motion black hole collides with couds of cosmic dusts these behave towards the black hole like a friction that raises by far the temperature of dusts with emission of electromagnetic radiations at very high energy.

\section{Black holes and binary stars}

With reference to fig.3, supposing that $\mathbf{V}$ is the speed of the bynary system and that the orbital speeds of the two stars are smaller than $c\left(v_{1}<c, v_{2}<c\right)$, the following cases can happen: 
a. The $\mathbf{V}$ speed is greater than $\mathrm{c}$ (speed of light and speed of quantum rays at high energy): $\mathrm{V}>\mathrm{C}$.

In figure the star binary system moves away with $\mathrm{V}$ speed from the observer, but the speed of each star with respect to the observer depends on the reciprocal position. In positions of figure star1 moves away with $\mathrm{V}-\mathrm{V}_{1}$ speed and star2 moves away with $\mathrm{V}+\mathrm{v}_{2}$ speed. In that event star2 certainly is a black hole while star1 is a black hole only if $V-v_{1}>c$.

b. The $\mathbf{V}$ speed is equal to $\mathrm{c}: \mathrm{V}=\mathrm{c}$.

In that event star2 is certainly a black hole and star1 isn't certainly a black hole in reciprocal positions of figure.

c. The $\mathbf{V}$ speed is smaller than $\mathrm{c}: \mathrm{V}<\mathrm{c}$. Always with reference to the situation in figure star1 is an ordinary star while star2 is a black hole if $V+v_{2}>c$.

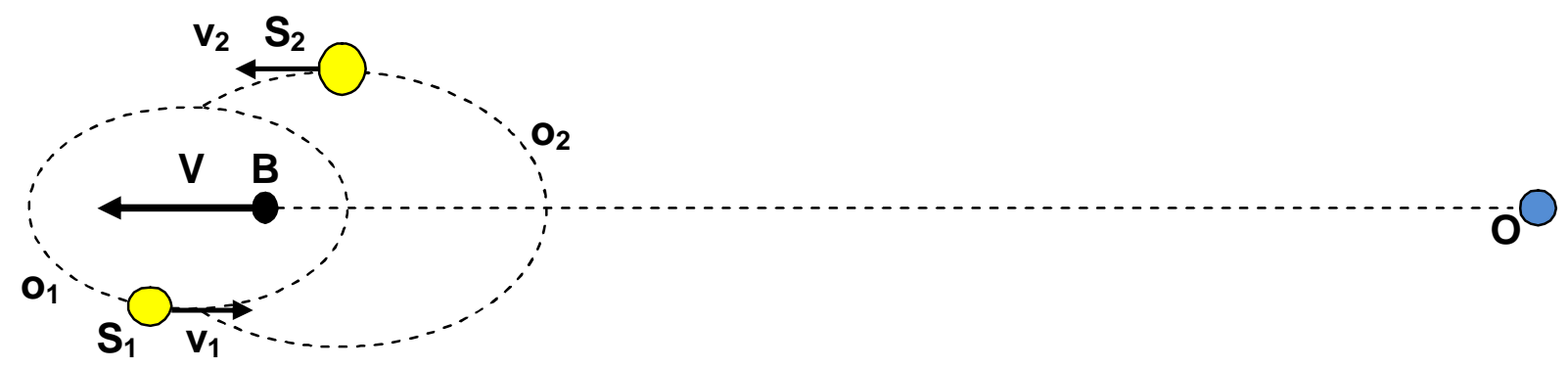

Fig.3 Graphic representation of a binary star. $\mathbf{o}_{1}$ is the orbit of star 1 and $\mathbf{o}_{2}$ is the orbit of star 2 .

$\mathbf{B}$ is the centre of mass of the binary star system which moves away from the observer with $\mathbf{V}$ speed. In the situation of figure, $\mathbf{v}_{1}$ is the approach orbital speed of the star1 with respect to the $\mathrm{O}$ observer and $\mathbf{v}_{2}$ is the departure orbital speed of the star2.

It is interesting to observe that in the relativistic theory the binary star system has a different behavior according to the reciprocal position between the two stars inside the binary system. Only astromical observations will be able to confirm this behavior.

The relativistic theory here described represents an alternative way with respect to the gravitational theory for explaining astronomical nature and physical behavior of black holes.

\section{References}

[1] D. Sasso, Not Linear Element, Cosmological Redshift and Deflection of Light in the Gravitational Field, viXra.org, id: 1111.0036, 2011

[2] S.J. Crothers, The Black Hole Catastrophe, viXra.org, id: 1111.0032, 2011

[3] D. Sasso, Relativistic Effects of the Theory of Reference Frames, Physics Essays (Physicsessays.com), Vol.20, No.1, 2007

[4] D. Sasso, Is the Speed of Light Invariant or Covariant?, viXra.org, id: 1003.2273, 2010 


\title{
Five-Dimensional Tangent Vectors in Space-Time IV. Generalization of Exterior Calculus
}

\author{
Alexander Krasulin \\ Institute for Nuclear Research of the Russian Academy of Sciences* \\ krasulin@post.com
}

\begin{abstract}
This part of the series is devoted to the generalization of exterior differential calculus. I give definition to the integral of a five-vector form over a limited space-time volume of appropriate dimension; extend the notion of the exterior derivative to the case of five-vector forms; and formulate the corresponding analogs of the generalized Stokes theorem and of the Poincare theorem about closed forms. I then consider the five-vector generalization of the exterior derivative itself; prove a statement similar to the Poincare theorem; define the corresponding five-vector generalization of flux; and derive the analog of the formula for integration by parts. I illustrate the ideas developed in this paper by reformulating the Lagrange formalism for classical scalar fields in terms of five-vector forms. In conclusion, I briefly discuss the five-vector analog of the Levi-Civita tensor and dual forms.
\end{abstract}

\section{A. Equivalence classes of two-, three- and four-dimensional volumes}

Consider a set $\Re_{2}$ of all smooth parametrized twodimensional surfaces going through a fixed space-time point $Q$. Let us lable these surfaces with calligraphic capital Roman letters: $\mathcal{A}, \mathcal{B}, \mathcal{C}$, etc. The two parameters of surface $\mathcal{A}$-its two inner coordinates - will be denoted as $\lambda_{\mathcal{A}}^{(1)}$ and $\lambda_{\mathcal{A}}^{(2)}$.

If $f$ is a real scalar function defined in the vicinity of $Q$, one can evaluate its derivatives at $Q$ relative to the parameters of a given surface $\mathcal{A}$ :

$$
\left[\frac{d f\left(P\left(\lambda_{\mathcal{A}}^{(1)}, \lambda_{\mathcal{A}}^{(2)}\right)\right)}{d \lambda_{\mathcal{A}}^{(k)}}\right]_{\lambda_{\mathcal{A}}^{(1)}=\lambda_{\mathcal{A}}^{(1)}(Q), \lambda_{\mathcal{A}}^{(2)}=\lambda_{\mathcal{A}}^{(2)}(Q)},
$$

where $k=1$ or 2 , and let us denote these derivatives as $\left.\partial_{\mathcal{A}}^{(k)} f\right|_{Q}$.

Let us focus our attention on the behaviour of twodimensional surfaces in the infinitesimal vicinity of $Q$. From that point of view $\Re_{2}$ can be divided into classes of equivalent surfaces that coincide in direction or in direction and parametrization. As in the case of parametrized curves, one can consider several degrees to which two given surfaces, $\mathcal{A}$ and $\mathcal{B}$, may coincide:

1. The two surfaces have the same direction at $Q$. A more precise formulation is the following: there exists a real $2 \times 2$ matrix, $\left\|a_{l}^{k}\right\|$, with a positive

\footnotetext{
*Former affiliation
}

determinant and such that for any scalar function $f$

$$
\left.\partial_{\mathcal{A}}^{(k)} f\right|_{Q}=\left.\sum_{l=1,2} a_{l}^{k} \partial_{\mathcal{B}}^{(l)} f\right|_{Q}
$$

where $k=1,2$.

2. The two surfaces have the same direction at $Q$; in the vicinity of $Q$ the direction of the corresponding inner coordinate lines is the same; and along these lines the corresponding parameters change with equal rates. More precisely: for any scalar function $f$

$$
\left.\partial_{\mathcal{A}}^{(k)} f\right|_{Q}=\left.\partial_{\mathcal{B}}^{(k)} f\right|_{Q},
$$

where $k=1,2$.

3. In the infinitesimal vicinity of $Q$, the two surfaces have the same direction and parametrization. This means that

$$
\lambda_{\mathcal{A}}^{(k)}(Q)=\lambda_{\mathcal{B}}^{(k)}(Q)
$$

and for any scalar function $f$

$$
\left.\partial_{\mathcal{A}}^{(k)} f\right|_{Q}=\left.\partial_{\mathcal{B}}^{(k)} f\right|_{Q},
$$

where $k=1,2$.

It is a simple matter to check that relations (1), (2) and (3) are all equivalence relations on $\Re_{2}$, and 
for each of them one can consider the corresponding quotient set-the set whose elements are classes of equivalent two-dimensional surfaces.

It is apparent that one can establish a one-toone correspondence between the equivalence classes of parametrized surfaces associated with relation (2) and ordered pairs of four-vectors, $\left(\mathbf{A}^{(1)}, \mathbf{A}^{(2)}\right)$, such that for any surface $\mathcal{A}$ from a given class

$$
\left.\partial_{\mathcal{A}}^{(k)} f\right|_{Q}=\left.\partial_{\mathbf{A}^{(k)}} f\right|_{Q} \quad(k=1,2)
$$

for any scalar function $f$. It is evident that the vectors in a pair should be linearly independent: otherwise the pair will not correspond to any nondegenerate surface.

In a similar manner one can establish a one-to-one correspondence between the classes of equivalent twosurfaces corresponding to relation (3) and ordered pairs of five-vectors, $\left(\mathbf{a}^{(1)}, \mathbf{a}^{(2)}\right)$, according to the formulae

$$
\left.\partial_{\mathcal{A}}^{(k)} f\right|_{Q}=\left.\partial_{\mathbf{a}^{(k)}} f\right|_{Q} \text { and } \lambda_{\mathcal{A}}^{(k)}=\lambda_{\mathbf{a}^{(k)}} \quad(k=1,2)
$$

for any surface $\mathcal{A}$ from a given class and for any scalar function $f$. In this case, besides being linearly independent, the five-vectors in a pair should satisfy one more requirement: neither of them should be an element of $\mathcal{E}$. Pairs where one of the five-vectors belongs to $\mathcal{E}$ can be considered as a special case corresponding to degenerate two-dimensional surfaces, which are lines.

In a similar manner one can consider a set $\Re_{3}$ of all smooth parametrized three-dimensional hypersurfaces going through a fixed space-time point $Q$ and a set $\Re_{4}$ of all parametrized four-dimensional volumes containing $Q$. If $f$ is a real scalar function defined in the vicinity of $Q$, one can evaluate its derivatives at $Q$ relative to the parameters $\lambda^{(k)}$ of a given hypersurface or four-volume $\mathcal{A}$, and I will denote these derivatives as $\left.\partial_{\mathcal{A}}^{(k)} f\right|_{Q}$ (here $k=1,2,3$ for a hypersurface and $k=1,2,3,4$ for a four-volume).

One can then focus one's attention on the behaviour of hypersurfaces and four-dimensional volumes in the infinitesimal vicinity of $Q$ and consider different degrees to which two given hypersurfaces or four-volumes may coincide. The analogs of relations (2) and (3) will have exactly the same form, only $k$ will now run 1 through 3 or 1 through 4 .

Finally, one can consider the quotient sets corresponding to the latter two equivalence relations and observe that it is possible to establish a one-to-one correspondence between their elements and triplets or quadruples of four- and five-vectors, respectively. The vectors in these triplets and quadruples should be linearly independent and, in addition, none of the five-vectors should belong to $\mathcal{E}$ unless the corresponding hypersurface or four-volume is degenerate.

\section{B. Integrals over m-dimensional volumes}

Let us first consider integrals over parametrized curves. Each of them can be viewed as a rule that assigns a certain number to every finite continuous parametrized curve within a certain region of spacetime. This number is additive and therefore can be presented as an integral

$$
\int_{\lambda_{a}}^{\lambda_{b}} d \lambda \phi(\lambda)
$$

where $\lambda_{a}$ and $\lambda_{b}$ are the end-point values of the curve parameter and $\phi(\lambda)$ is a certain numerical function, which may depend on the curve direction in the infinitesimal vicinity of the integration point.

In applications one is usually interested in invariant integrals, whose value is independent of the curve parametrization. This is impossible if $\phi$ in formula (4) depends only on the integration point (if there exists a scalar function $f$ such that $\phi(\lambda)=f(P(\lambda))$ for every curve), so the dependence on the curve direction is quite essential. This dependence can be of different types. Within four-vector exterior calculus one considers integrals where $\phi(\lambda)$ is a linear function of the tangent four-vector $\mathbf{U}(\lambda)$ and therefore can be presented as a contraction of $\mathbf{U}(\lambda)$ with some fourvector 1 -form, $\widetilde{\mathbf{S}}$, defined in some region of space-time containing the curve:

$$
\phi(\lambda)=<\widetilde{\mathbf{S}}(P(\lambda)), \mathbf{U}(\lambda)>.
$$

Such an integral is denoted simply as $\int \widetilde{\mathbf{S}}$ and is referred to as the integral of 1-form $\widetilde{\mathbf{S}}$ along the specified curve.

In a similar way one can consider integrals over limited two-, three- and four-dimensional volumes. Each of them can be presented in the form

$$
\int_{\Lambda} d \lambda^{(1)} \ldots d \lambda^{(m)} \phi\left(\lambda^{(1)}, \ldots, \lambda^{(m)}\right),
$$

where $m=2,3$, or $4 ; \Lambda$ is the range of variation of inner coordinates; and $\phi\left(\lambda^{(1)}, \ldots, \lambda^{(m)}\right)$ is a certain numerical function, which may depend on the direction of the given $m$-dimensional surface in the infinitesimal vicinity of the integration point. Within four-vector exterior calculus one deals with integrals whose integrand is a linear function of each of the tangent four-vectors $\mathbf{U}^{(k)}(k=1, \ldots, m)$ that correspond to the selected surface parameters. It is easy to show 
that for such an integral to be invariant, the function $\phi$ has to be antisymmetric with respect to permutations of $\mathbf{U}^{(1)}, \ldots, \mathbf{U}^{(m)}$. It therefore can be presented as a contraction of the multivector $\mathbf{U}^{(1)} \wedge \ldots \wedge \mathbf{U}^{(m)}$ with some four-vector $m$-form $\widetilde{\mathbf{S}}$ :

$$
\phi=<\widetilde{\mathbf{S}}, \mathbf{U}^{(1)} \wedge \ldots \wedge \mathbf{U}^{(m)}>.
$$

Such an integral is referred to as the integral of $m$ form $\widetilde{\mathbf{S}}$ over the specified $m$-dimensional surface.

We thus see that in those cases where a given $m^{-}$ dimensional surface is considered only as an integration volume for the integrals discussed above, it is more adequate to characterize its local direction and parametrization with the multivector $\mathbf{U}^{(1)} \wedge \ldots \wedge$ $\mathbf{U}^{(m)}$ rather than with the set of $m$ individual tangent four-vectors. One can then consider the corresponding equivalence relation, according to which two nondegenerate parameterized $m$-dimensional surfaces going through a given point $Q$ belong to the same equivalence class if and only if their multivectors coincide. It is not difficult to see that this relation is equivalent to relation (1) (and to its analogs for three- and four-dimensional volumes) with the additional requirement that the matrix $\left\|a_{l}^{k}\right\|$ be unimodular. One should also notice that at $m=1$ this equivalence relation reproduces relation (2) of part II for parametrized curves.

Let us now see what will happen if the integration volume is characterized by tangent five-vectors. Since we are generalizing exterior calculus, it is natural to consider the case where the integrand is a linear function of each of these five-vectors.

Let us, again, start with parametrized curves. Owing to the invariant decomposition of $V_{5}$ into the direct sum of $\mathcal{Z}$ and $\mathcal{E}$, any integral of the considered kind can be presented as

$$
\int_{\lambda_{a}}^{\lambda_{b}} d \lambda<\widetilde{\mathbf{s}}, \mathbf{u}^{\mathcal{Z}}>+\int_{\lambda_{a}}^{\lambda_{b}} d \lambda<\widetilde{\mathbf{s}}^{\prime}, \mathbf{u}^{\mathcal{E}}>
$$

where $\mathbf{u}(\lambda)$ is the tangent five-vector and $\widetilde{\mathbf{s}}$ and $\widetilde{\mathbf{s}}^{\prime}$ are some five-vector 1 -forms. The first term in this formula is an invariant integral, whose value coincides with that of the integral $\int \widetilde{\mathbf{S}}$ along the same curve, where $\widetilde{\mathbf{S}}$ is the four-vector 1 -form that corresponds to $\widetilde{\mathbf{s}}$. The second term in formula (5) is proportional to

$$
\int_{\lambda_{a}}^{\lambda_{b}} \lambda d \lambda<\widetilde{\mathbf{s}}^{\prime}, \mathbf{1}>
$$

and is not an invariant integral unless $\left\langle\widetilde{\mathbf{s}}^{\prime}, \mathbf{1}\right\rangle$ is identically zero. Therefore, any invariant integral of the considered type should have the form of the first term in formula (5). The latter will be referred to as the integral of five-vector 1-form $\widetilde{\mathbf{s}}$ along the specified curve.

In a similar way one can deal with invariant integrals over two-, three- and four-dimensional volumes. One can show that each of them can be presented in the form

$$
\int_{\Lambda} d \lambda^{(1)} \ldots d \lambda^{(m)}<\widetilde{\mathbf{s}},\left(\mathbf{u}^{(1)}\right)^{\mathcal{Z}} \wedge \ldots \wedge\left(\mathbf{u}^{(m)}\right)^{\mathcal{Z}}>,
$$

where $m=2,3$, or 4 and $\widetilde{\mathbf{s}}$ is now some five-vector $m$ form. Integral (6) will be denoted as $\int \widetilde{\mathbf{s}}$ and will be referred to as the integral of $m$-form $\widetilde{\mathbf{s}}$ over the specified $m$-dimensional surface. As one can see, only the $\widetilde{\mathcal{Z}}$-component of $\widetilde{\mathbf{s}}$ gives contribution to the integral, so for any integration volume the value of the latter coincides with that of the integral $\int \widetilde{\mathbf{S}}$, where $\widetilde{\mathbf{S}}$ is the four-vector $m$-form that corresponds to $\widetilde{\mathbf{S}}^{\mathcal{Z}}$.

There exists another interesting way of constructing invariant integrals. For that one should consider the given $m$-dimensional integration surface (now $m$ $=1,2,3$ or 4 ) as a degenerate volume of dimension $m+1$ and characterize it with the multivector $\mathbf{u}^{(1)} \wedge \ldots \wedge \mathbf{u}^{(m)} \wedge \mathbf{e}$, where $\mathbf{u}^{(k)}$ are the five-dimensional tangent vectors that correspond to nondegenerate inner coordinates and $\mathbf{e}$ is a nonzero five-vector from $\mathcal{E}$. The integral itself will have the form

$$
\int_{\Lambda} d \lambda^{(1)} \ldots d \lambda^{(m)}<\widetilde{\mathbf{t}}, \mathbf{u}^{(1)} \wedge \ldots \wedge \mathbf{u}^{(m)} \wedge \mathbf{e}>
$$

where $\widetilde{\mathbf{t}}$ is some five-vector form of rank $m+1$.

One should now select the five-vector $\mathbf{e}$ from $\mathcal{E}$. It is evident that allowing e to vary from one point to another is equivalent to introducing a certain weight factor into the integral. As a rule, such factors are not considered, but even if one does introduce one, it is more convenient not to absorb it into the multivector, so that the role of the latter would consist only in specifying the infinitesimal integration volume, as it does in ordinary exterior calculus. Considering this, it will be taken that $\mathbf{e}$ is a constant vector.

For the same reason one can choose e to have any nonzero size. If one wishes the five-vector exterior calculus to be applicable to manifolds without metric and without affine connection (as is its four-vector analog), one should select the size of e without any reference to the inner product nor to parallel transport, and the only distinguished choice is then $\mathbf{e}=\mathbf{1}$.

Since $\mathbf{u}^{(1)} \wedge \ldots \wedge \mathbf{u}^{(m)} \wedge \mathbf{1}=\left(\mathbf{u}^{(1)}\right)^{\mathcal{Z}} \wedge \ldots \wedge\left(\mathbf{u}^{(m)}\right)^{\mathcal{Z}} \wedge \mathbf{1}$, for any integration volume integral (7) will have the same value as integral (6) in which $\widetilde{\mathbf{s}}$ is defined by the condition

$$
\begin{aligned}
<\widetilde{\mathbf{s}},\left(\mathbf{u}^{(1)}\right)^{\mathcal{Z}} & \wedge \ldots \wedge\left(\mathbf{u}^{(m)}\right)^{\mathcal{Z}}> \\
& =<\widetilde{\mathbf{t}}, \mathbf{u}^{(1)} \wedge \ldots \wedge \mathbf{u}^{(m)} \wedge \mathbf{1}>.
\end{aligned}
$$


One should also notice that contrary to the case of integral (6), the contribution to integral (7) is given by the $\widetilde{\mathcal{E}}$-component of form $\widetilde{\mathbf{t}}$.

We thus see that a given five-vector $m$-form $\widetilde{\mathbf{s}}(m=$ $1,2,3$ or 4 ) can be integrated over volumes of two different dimensions: $(i)$ over an $m$-dimensional surface, in which case the integral is determined only by the $\widetilde{\mathcal{Z}}$-component of $\widetilde{\mathbf{s}}$, or $(i i)$ over an $(m-1)$-dimensional surface, in which case the integral depends only on the $\widetilde{\mathcal{E}}$-component of $\widetilde{\mathbf{s}}$. At $m=1$ the integration volume in the second case degenerates into an isolated point (or several isolated points), and the integral is replaced by the value of the contraction of the given 1-form with the five-vector 1 . Five-vector 5 -forms can be integrated over volumes of only one dimension: four. For an obvious reason, they do not have a $\widetilde{\mathcal{Z}}$-component.

From our analysis it also follows that each additive rule for assigning numbers to limited $m$-dimensional volumes $(m=1,2,3$ or 4$)$ produced by the integrals considered in this section can be interpreted in three different ways. It can be regarded as an integral of a four-vector $m$-form; or as an integral of a five-vector $m$-form; or as an integral of a five-vector $(m+1)$-form, over the considered volume. The three mentioned forms - which have been denoted above as $\widetilde{\mathbf{S}}, \widetilde{\mathbf{s}}$, and $\widetilde{\mathbf{t}}$, respectively - are related to one another in the following way:

$$
\begin{aligned}
<\widetilde{\mathbf{S}}, \mathbf{U}^{(1)} & \wedge \ldots \wedge \mathbf{U}^{(m)}> \\
& =<\widetilde{\mathbf{s}},\left(\mathbf{u}^{(1)}\right)^{\mathcal{Z}} \wedge \ldots \wedge\left(\mathbf{u}^{(m)}\right)^{\mathcal{Z}}> \\
& =<\widetilde{\mathbf{t}}, \mathbf{u}^{(1)} \wedge \ldots \wedge \mathbf{u}^{(m)} \wedge \mathbf{1}>
\end{aligned}
$$

where $\mathbf{u}^{(k)} \in \mathbf{U}^{(k)}$ for $k=1, \ldots, m$. This invariant relation between forms of different types exists at each $m$ owing to the isomorphicity of the three corresponding vector spaces: (1) the space of multivectors of rank $m$ made out of four-vectors; (2) the space of multivectors of rank $m$ made out of fivevectors from $\mathcal{Z}$; and (3) the space of wedge products of $\mathbf{1}$ with multivectors of rank $m$ made out of five-vectors. At $m=1$ these three vector spaces are respectively $V_{4}, \mathcal{Z}$, and the maximal vector space of simple bivectors over $V_{5}$ with the directional vector from $\mathcal{E}$, the isomorphisms between which have already been discussed in part II. In view of the mentioned isomorphicity, the equivalence relation between parametrized $m$-dimensional volumes obtained by equating the corresponding multivectors is exactly the same for all three types of multivectors considered above.

\section{Generalized Stokes theorem}

Let $\partial V$ be the closed $m$-dimensional boundary of an $(m+1)$-dimensional surface $V$ and let $\widetilde{\mathbf{S}}$ be a fourvector $m$-form defined throughout $V$. The integral of $\widetilde{\mathbf{S}}$ over $\partial V$, which will be denoted as

$$
\oint_{\partial V} \widetilde{\mathbf{S}}
$$

is called the flux of form $\widetilde{\mathbf{S}}$ through the closed surface $\partial V$. The generalized Stokes theorem states that this flux equals the integral over the interior of $V$ of a certain $(m+1)$-form, which is denoted as $\mathbf{d} \widetilde{\mathbf{S}}$ and is called the exterior derivative of $\widetilde{\mathbf{S}}$ :

$$
\oint_{\partial V} \widetilde{\mathbf{S}}=\int_{V} \mathbf{d} \widetilde{\mathbf{S}}
$$

One can give several equivalent definitions to the exterior derivative. As a first step, one usually defines it for a scalar function (regarded as a four-vector 0form): $\mathbf{d} f$ is such a four-vector 1 -form that

$$
<\mathbf{d} f, \mathbf{U}>=\partial_{\mathbf{U}} f
$$

for any four-vector $\mathbf{U}$. This enables one to present the basis 1-forms dual to a coordinate four-vector basis associated with coordinates $x^{\alpha}$ as exterior derivatives $\mathbf{d} x^{\alpha}$. One can then define the effect of $\mathbf{d}$ on an arbitrary form in the following way: for the $m$-form

$$
\widetilde{\mathbf{S}}=S_{\left|\alpha_{1} \ldots \alpha_{m}\right|} \mathbf{d} x^{\alpha_{1}} \wedge \ldots \wedge \mathbf{d} x^{\alpha_{m}}
$$

one has

$$
\mathbf{d} \widetilde{\mathbf{S}}=\mathbf{d} S_{\left|\alpha_{1} \ldots \alpha_{m}\right|} \wedge \mathbf{d} x^{\alpha_{1}} \wedge \ldots \wedge \mathbf{d} x^{\alpha_{m}} .
$$

Another, equivalent definition of the exterior

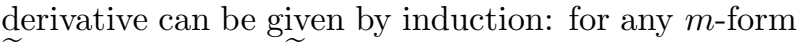
$\widetilde{\mathbf{S}}$ and any $n$-form $\widetilde{\mathbf{T}}$

$$
\mathbf{d}(\widetilde{\mathbf{S}} \wedge \widetilde{\mathbf{T}})=\mathbf{d} \widetilde{\mathbf{S}} \wedge \widetilde{\mathbf{T}}+(-1)^{m} \widetilde{\mathbf{S}} \wedge \mathbf{d} \widetilde{\mathbf{T}}
$$

and for any form $\widetilde{\mathbf{S}}$

$$
\operatorname{dd} \widetilde{\mathbf{S}}=\mathbf{0} .
$$

One can also define the exterior derivative of any four-vector form à la equation (10): if $\widetilde{\mathbf{S}}$ is a 1 -form, then $\mathbf{d} \widetilde{\mathbf{S}}$ is such a 2 -form that

$$
\begin{aligned}
<\mathbf{d} \widetilde{\mathbf{S}}, \mathbf{U} \wedge & \mathbf{V}>=\partial_{\mathbf{U}}<\widetilde{\mathbf{S}}, \mathbf{V}> \\
& -\partial_{\mathbf{V}}<\widetilde{\mathbf{S}}, \mathbf{U}>-<\widetilde{\mathbf{S}},[\mathbf{U}, \mathbf{V}]>
\end{aligned}
$$

for any four-vector fields $\mathbf{U}$ and $\mathbf{V}$; if $\widetilde{\mathbf{S}}$ is a 2 -form, then $\mathbf{d} \widetilde{\mathbf{S}}$ is such a 3 -form that

$$
\begin{aligned}
& <\mathbf{d} \widetilde{\mathbf{S}}, \mathbf{U} \wedge \mathbf{V} \wedge \mathbf{W}> \\
& =\partial_{\mathbf{U}}<\widetilde{\mathbf{S}}, \mathbf{V} \wedge \mathbf{W}>+\partial_{\mathbf{V}}<\widetilde{\mathbf{S}}, \mathbf{W} \wedge \mathbf{U}> \\
& +\partial_{\mathbf{W}}<\widetilde{\mathbf{S}}, \mathbf{U} \wedge \mathbf{V}>-<\widetilde{\mathbf{S}},[\mathbf{U}, \mathbf{V}] \wedge \mathbf{W}> \\
& -<\widetilde{\mathbf{S}},[\mathbf{V}, \mathbf{W}] \wedge \mathbf{U}>-<\widetilde{\mathbf{S}},[\mathbf{W}, \mathbf{U}] \wedge \mathbf{V}>
\end{aligned}
$$


for any four-vector fields $\mathbf{U}, \mathbf{V}$ and $\mathbf{W}$; etc.

Let us now generalize the concept of flux and the Stokes theorem to the case of five-vector forms.

It is natural to define the flux of a five-vector $m$ form $\widetilde{\mathbf{t}}$ through a closed $m$-dimensional surface $\partial V$ as the integral of $\widetilde{\mathbf{t}}$ over $\partial V$, and I will denote this particular integral as

$$
\oint_{\partial V} \tilde{\mathbf{t}}
$$

According to section $\mathrm{B}$, this flux equals the flux through $\partial V$ of the four-vector $m$-form $\widetilde{\mathbf{T}}$ corresponding to $\widetilde{\mathbf{t}} \widetilde{\mathcal{Z}}$. If $\partial V$ is the boundary of the $(m+1)$ dimensional volume $V$, then by virtue of the generalized Stokes theorem for four-vector forms, one has

$$
\oint_{\partial V} \widetilde{\mathbf{t}}=\oint_{\partial V} \widetilde{\mathbf{T}}=\int_{V} \mathbf{d} \widetilde{\mathbf{T}} .
$$

Now, using the one-to-one correspondence that exists between four-vector forms and $\widetilde{\mathcal{Z}}$-components of fivevector forms, one can present the flux of $\widetilde{\mathbf{t}}$ through $\partial V$ as an integral over the interior of $V$ of a five-vector $(m+1)$-form whose $\widetilde{\mathcal{Z}}$-component corresponds to $\mathbf{d} \widetilde{\mathbf{T}}$. It is natural to call this form the exterior derivative of $\widetilde{\mathbf{t}}$ and to denote it as $\mathbf{d} \widetilde{\mathbf{t}}$. We thus obtain the following variant of the generalized Stokes theorem for five-vector forms:

$$
\oint_{\partial V} \widetilde{\mathbf{t}}=\int_{V} \mathbf{d} \widetilde{\mathbf{t}}
$$

where in both integrals the rank of the form equals the dimension of the integration volume.

It is not difficult to show that if

$$
\widetilde{\mathbf{t}}=t_{\left|A_{1} \ldots A_{m}\right|} \widetilde{\mathbf{o}}^{A_{1}} \wedge \ldots \wedge \widetilde{\mathbf{o}}^{A_{m}},
$$

where $\widetilde{\mathbf{o}}^{A}$ is the basis of five-vector 1-forms dual to a passive regular coordinate five-vector basis, then

$$
(\mathbf{d} \widetilde{\mathbf{t}})^{\widetilde{\mathcal{Z}}}=\partial_{\alpha} t_{\left|\alpha_{1} \ldots \alpha_{m}\right|} \widetilde{\mathbf{o}}^{\alpha} \wedge \widetilde{\mathbf{o}}^{\alpha_{1}} \wedge \ldots \wedge \widetilde{\mathbf{o}}^{\alpha_{m}} .
$$

The $\widetilde{\mathcal{E}}$-component of $\mathbf{d} \widetilde{\mathbf{t}}$ is not fixed by the above correspondence, and to determine it one should consider the flux of $\widetilde{\mathbf{t}}$ through a closed surface of dimension $m-1$. Let us denote this surface, again, as $\partial V$. The flux in question will then be the integral of $\widetilde{\mathbf{t}}$ over $\partial V$. According to section $\mathrm{B}$, it equals the flux through $\partial V$ of the five-vector $(m-1)$-form $\widetilde{\mathbf{s}}$ related to $\widetilde{\mathbf{t}}$ by an equation similar to equation (8). If $V$ denotes an $m$ dimensional volume limited by $\partial V$, then according to the variant of the generalized Stokes theorem we have obtained above, one has

$$
\oint_{\partial V} \widetilde{\mathbf{t}}=\oint_{\partial V} \widetilde{\mathbf{s}}=\int_{V} \mathbf{d} \widetilde{\mathbf{s}} .
$$

If now one uses the one-to-one correspondence between the $\widetilde{\mathcal{Z}}$-components of five-vector $m$-forms and the $\widetilde{\mathcal{E}}$-components of five-vector $(m+1)$-forms, one can present the flux of $\widetilde{\mathbf{t}}$ through $\partial V$ as an integral over the interior of $V$ of a five-vector $(m+1)$ form whose $\widetilde{\mathcal{E}}$-component corresponds to $(\mathbf{d} \widetilde{\mathbf{s}})^{\mathcal{Z}}$. This $(m+1)$-form also has the meaning of an exterior derivative of $\widetilde{\mathbf{t}}$, and since its $\widetilde{\mathcal{Z}}$-component is not fixed by the above correspondence, one can take that this is the same form $\mathbf{d} \widetilde{\mathbf{t}}$ we have introduced above. We thus obtain another variant of the generalized Stokes theorem, which has the same form as equation (15), only now in both integrals the rank of the form is one unit greater than the dimension of the integration volume. It is not difficult to show that

$$
(\mathbf{d} \widetilde{\mathbf{t}})^{\widetilde{\mathcal{E}}}=\partial_{\alpha} t_{\left|\alpha_{1} \ldots \alpha_{m-1}\right| 5} \widetilde{\mathbf{o}}^{\alpha} \wedge \widetilde{\mathbf{o}}^{\alpha_{1}} \wedge \ldots \wedge \widetilde{\mathbf{o}}^{\alpha_{m-1}} \wedge \widetilde{\mathbf{o}}^{5}
$$

which together with equation (17) gives one the following general formula for calculating the exterior derivative of $m$-form (16):

$$
\mathbf{d} \widetilde{\mathbf{t}}=\partial_{A} t_{\left|A_{1} \ldots A_{m}\right|} \widetilde{\mathbf{o}}^{A} \wedge \widetilde{\mathbf{o}}^{A_{1}} \wedge \ldots \wedge \widetilde{\mathbf{o}}^{A_{m}} .
$$

As in four-vector exterior calculus, one can define the exterior derivative of a five-vector form without any reference to the Stokes theorem, i.e. as a certain operator that produces an $(m+1)$-form out of an $m$ form. One should, again, start with a scalar function $f$, which is now regarded as a five-vector 0-form, and define its exterior derivative as such a five-vector 1form $\mathbf{d} f$ that

$$
<\mathbf{d} f, \mathbf{u}>=\partial_{\mathbf{u}} f
$$

for any five-vector $\mathbf{u}$. One can then present formula (18) in a form similar to equation (12):

$$
\mathbf{d \mathbf { t }}=\mathbf{d} t_{\left|A_{1} \ldots A_{m}\right|} \wedge \widetilde{\mathbf{o}}^{A_{1}} \wedge \ldots \wedge \widetilde{\mathbf{o}}^{A_{m}}
$$

and take this to be the definition of $\widetilde{\mathbf{d}} \widetilde{\mathbf{t}}$.

Another way of defining the exterior derivative for five-vector forms is similar to equations (14): if $\widetilde{\mathbf{t}}$ is a 1-form, then $\mathbf{d} \widetilde{\mathbf{t}}$ is such a 2 -form that

$$
\begin{aligned}
<\mathbf{d} \widetilde{\mathbf{t}}, \mathbf{u} \wedge \mathbf{v}>=\partial_{\mathbf{u}}<\widetilde{\mathbf{t}}, \mathbf{v}> \\
-\partial_{\mathbf{v}}<\widetilde{\mathbf{t}}, \mathbf{u}>-<\widetilde{\mathbf{t}},[\mathbf{u}, \mathbf{v}]>
\end{aligned}
$$

for any two five-vector fields $\mathbf{u}$ and $\mathbf{v}$; etc.

Finally, from formula (20) one can derive the analogs of equations (13): for any $m$-form $\widetilde{\mathbf{s}}$ and any $n$-form $\widetilde{\mathbf{t}}$

$$
\mathbf{d}(\widetilde{\mathbf{s}} \wedge \widetilde{\mathbf{t}})=\mathbf{d} \widetilde{\mathbf{s}} \wedge \widetilde{\mathbf{t}}+(-1)^{m} \widetilde{\mathbf{s}} \wedge \mathbf{d} \widetilde{\mathbf{t}}
$$

and for any form $\widetilde{\mathbf{t}}$

$$
\mathbf{d d} \widetilde{\mathbf{t}}=\mathbf{0} .
$$


However, unlike the case of four-vector forms, the latter two equations (together with equation (19)) are not enough to define the exterior derivative completely. To gain a better understanding of this fact, one should recall how things work out in the case of four-vector forms.

From the generalized Stokes theorem one obtains the following formula for the exterior derivative:

$$
\mathbf{d} \widetilde{\mathbf{S}}=\mathbf{d} S_{\left|\alpha_{1} \ldots \alpha_{m}\right|} \wedge \widetilde{\mathbf{O}}^{\alpha_{1}} \wedge \ldots \wedge \widetilde{\mathbf{O}}^{\alpha_{m}}
$$

where $\widetilde{\mathbf{O}}^{\alpha}$ is any basis of four-vector 1-forms dual to a coordinate basis. Provided the effect of $\mathbf{d}$ on a scalar function is known, equation (23) is equivalent to equation (13a) and the requirement

$$
\mathbf{d} \widetilde{\mathbf{O}}^{\alpha}=0
$$

(the latter, by the way, is a necessary and sufficient condition of the corresponding basis of four-vectors being a coordinate basis). From equation (10) one can derive that $\widetilde{\mathbf{O}}^{\alpha}$ equal $\mathbf{d} x^{\alpha}$, and so equation (24) follows from equation (13b) and can be replaced with it.

In the case of five-vector forms, equation (20) is equivalent to equation (22a) and the requirement

$$
\mathbf{d} \widetilde{\mathbf{o}}^{A}=0,
$$

which is a necessary condition of the corresponding basis of five-vectors being a regular coordinate basis. However, from equation (19) one can only derive that $\widetilde{\mathbf{o}}^{\alpha}=\mathbf{d} x^{\alpha}$. The fifth basis 1-form, which in this case equals $\widetilde{\mathbf{j}}$, cannot be presented as an exterior derivative of any 0-form (this is also true of any basis of fivevector 1 -forms dual to a standard basis). Thus, its exterior derivative is not determined by the rule $\mathbf{d d}=$ 0 , and to make equations (22) equivalent to equation (20) one should supplement the former with a third equation:

$$
\mathbf{d} \mathbf{j}=0 \text {. }
$$

The fact that basis four-vector 1-forms dual to a coordinate basis can be presented as exterior derivatives of scalar functions, whereas in the case of fivevector forms this is possible only for the first four basis 1-forms, is closely related to the Poincare theorem in application to four-vector forms and to its analog for forms associated with five-vectors. The Poincare theorem states that each four-vector $m$-form $\widetilde{\mathbf{S}}$ with $m \geq 1$, which in a certain region of space-time ${ }^{1}$ satisfies the equation $\mathbf{d} \widetilde{\mathbf{S}}=0$ (such forms are called closed), can be presented in this region as an exterior

\footnotetext{
${ }^{1}$ This region should satisfy certain requirements, which can be found e.g. in ref.[1].
}

derivative of some four-vector $(m-1)$-form. In agreement with this theorem, the basis four-vector 1-forms $\widetilde{\mathbf{O}}^{\alpha}$, which satisfy equation (24), can be presented as exterior derivatives of certain scalar functions (which in this particular case can be chosen to coincide with coordinates). A statement similar to the Poincare theorem can be easily shown to hold for forms corresponding to five-vectors:

Any five-vector $m$-form $\widetilde{\mathbf{s}}$ with $m \geq 2$, which in a certain region of space-time (subject to the same constraints that are imposed within the Poincare theorem for four-vector forms) satisfies the equation $\mathbf{d} \widetilde{\mathbf{s}}=0$, can be presented in this region as an exterior derivative of some five-vector $(m-1)$-form.

At $m=1$ the theorem works for the $\widetilde{\mathcal{Z}}$-component only. The $\widetilde{\mathcal{E}}$-component of any five-vector 1 -form, if it is nonzero, is not an exterior derivative of any scalar function; in this case from $\mathbf{d}\left(\widetilde{\mathbf{s}}^{\widetilde{\mathcal{E}}}\right)=\mathbf{0}$ follows $\widetilde{\mathbf{s}}=$ const $\cdot \widetilde{\mathbf{j}}$. One can easily see how this theorem manifests itself in the case of basis five-vector 1-forms $\widetilde{\mathbf{o}}^{A}$, which satisfy equation (25).

The representation of basis four-vector 1-forms $\widetilde{\mathbf{O}}^{\alpha}$ as exterior derivatives of coordinates is a convenient way of indicating that the selected basis of 1-forms is dual to a coordinate basis. We see that no similar convenient representation exists in the case of fivevector forms, so one should either introduce a special notation for the basis of 1-forms dual to a regular coordinate basis or each time indicate explicitly what kind of a basis is used in the formulae presented.

\section{Five-vector exterior derivative}

In the previous section we have generalized the concept of flux and of exterior derivative to the case of five-vector forms. It turns out that one can go farther and consider an operator similar to the exterior derivative but which differs from the latter in that $\partial$ in the right-hand side of formula (18) is replaced with its five-vector counterpart: $\partial+\lambda \cdot \varsigma \cdot \mathbf{1}$. Let us call this operator the five-vector exterior derivative and denote its effect on a five-vector form $\widetilde{\mathbf{t}}$ as $\widetilde{\mathbf{d}}$.

Since the operator $\partial_{\mathbf{u}}+\lambda_{\mathbf{u}} \cdot \varsigma \cdot \mathbf{1}$ will often appear in the following formulae, it is convenient to introduce a special notation for it: $\partial_{\mathbf{u}}$. By definition, for any scalar function $f$,

$$
\partial_{\mathbf{u}} f \equiv \mathbf{u}[f]=\partial_{\mathbf{u}} f+\varsigma \lambda_{\mathbf{u}} f
$$

From equations (13) of part II it follows that the effect of $\boldsymbol{\partial}_{\mathbf{u}}$ on the product of two scalar functions is 
formally described by the rule

$$
\partial_{\mathbf{u}}(f g)=\boldsymbol{\partial}_{\mathbf{u}} f \cdot g+f \cdot \partial_{\mathbf{u}} g-\boldsymbol{\partial}_{\mathbf{u}} 1 \cdot f g,
$$

where 1 is the constant unity function. As in the case of operators $\partial$ and $\nabla$, it is convenient to introduce the notation $\boldsymbol{\partial}_{A} \equiv \boldsymbol{\partial}_{\mathbf{e}_{A}}$, where $\mathbf{e}_{A}$ is the selected basis of five-vectors. Using this notation, one can present the five-vector exterior derivative of $m$-form (16) as

$$
\mathbf{d} \tilde{\mathbf{t}}=\boldsymbol{\partial}_{A} t_{\left|A_{1} \ldots A_{m}\right|} \widetilde{\mathbf{o}}^{A} \wedge \widetilde{\mathbf{o}}^{A_{1}} \wedge \ldots \wedge \widetilde{\mathbf{o}}^{A_{m}},
$$

where, let us recall, $\widetilde{\mathbf{o}}^{A}$ is the basis of five-vector 1forms dual to a passive regular coordinate basis. One can easily see that there exists a very simple relation between $\mathbf{d}$ and $\mathbf{d}$ : for any form $\widetilde{\mathbf{t}}$

$$
\mathbf{d} \widetilde{\mathbf{t}}=\mathbf{d} \tilde{\mathbf{t}}+\widetilde{\mathbf{j}} \wedge \widetilde{\mathbf{t}}
$$

From equation (29) it follows that the effect of $\mathbf{d}$ on a scalar function $f$ (regarded as a five-vector 0-form) is given by the formula

$$
<\mathbf{d} f, \mathbf{u}>=\boldsymbol{\partial}_{\mathbf{u}} f
$$

which is the analog of equations (10) and (19) for $\mathbf{d}$. One can then present formula (29) in a form similar to equations (20) and (23):

$$
\mathbf{d} \widetilde{\mathbf{t}}=\mathbf{d} t_{\left|A_{1} \ldots A_{m}\right|} \wedge \widetilde{\mathbf{o}}^{A_{1}} \wedge \ldots \wedge \widetilde{\mathbf{o}}^{A_{m}}
$$

which, together with formula (31), can serve as a definition of the effect of $\mathbf{d}$ on an arbitrary five-vector form.

Another way of defining the five-vector exterior derivative is similar to equations (14) and (21): if $\widetilde{\mathbf{t}}$ is a five-vector 1 -form, then $\mathbf{d} \widetilde{\mathbf{t}}$ is such a 2 -form that

$$
\begin{aligned}
<\mathbf{d} \widetilde{\mathbf{t}}, \mathbf{u} \wedge \mathbf{v}>=\boldsymbol{\partial}_{\mathbf{u}}<\widetilde{\mathbf{t}}, \mathbf{v}> \\
-\boldsymbol{\partial}_{\mathbf{v}}<\widetilde{\mathbf{t}}, \mathbf{u}>-<\widetilde{\mathbf{t}},[\mathbf{u}, \mathbf{v}]>
\end{aligned}
$$

for any two five-vector fields $\mathbf{u}$ and $\mathbf{v}$; etc.

Finally, from formula (32) one can derive the analogs of equations (13) and (22): for any $m$-form $\widetilde{\mathbf{s}}$ and any $n$-form $\widetilde{\mathbf{t}}$

$$
\mathbf{d}(\widetilde{\mathbf{s}} \wedge \widetilde{\mathbf{t}})=\mathbf{d} \widetilde{\mathbf{s}} \wedge \widetilde{\mathbf{t}}+(-1)^{m} \widetilde{\mathbf{s}} \wedge \mathbf{d} \widetilde{\mathbf{t}}-\mathbf{d} 1 \wedge \widetilde{\mathbf{s}} \wedge \widetilde{\mathbf{t}},
$$

and for any form $\widetilde{\mathbf{t}}$

$$
\mathbf{d d} \widetilde{\mathbf{t}}=\mathbf{0} \text {. }
$$

These two equations are equivalent to equation (32) and, together with equation (31), can be used to define the effect of $\mathbf{d}$ on any five-vector form by induction. Indeed, by using equation (31) one can show that any basis of five-vector 1 -forms, $\widetilde{\mathbf{o}}^{A}$, dual to a passive regular coordinate basis associated with coordinates $x^{\alpha}$ can be presented as

$$
\left\{\begin{array}{l}
\widetilde{\mathbf{o}}^{\alpha}=\mathbf{d} x^{\alpha}-x^{\alpha} \mathbf{d} 1 \\
\widetilde{\mathbf{o}}^{5}=\mathbf{d} 1
\end{array} .\right.
$$

From equations (34) one then obtains that

$$
\mathbf{d} \widetilde{\mathbf{o}}^{A}-\mathbf{d} 1 \wedge \widetilde{\mathbf{o}}^{A}=\mathbf{0},
$$

which is nothing but equation (25) expressed in terms of $\mathbf{d}$. By induction, from the latter equation one can derive that

$$
\mathbf{d}\left(\widetilde{\mathbf{o}}^{A_{1}} \wedge \ldots \wedge \widetilde{\mathbf{o}}^{A_{m}}\right)-\mathbf{d} 1 \wedge \widetilde{\mathbf{o}}^{A_{1}} \wedge \ldots \wedge \widetilde{\mathbf{o}}^{A_{m}}=\mathbf{0}
$$

for any $m \geq 1$, which together with equation (34a) is equivalent to equation (32).

It is evident that the analog of the Poincare theorem for five-vector forms presented in the previous section can be reformulated in terms of $\boldsymbol{d}$ in the following way: any five-vector $m$-form with $m \geq 2$ or 1 -form from $\widetilde{\mathcal{Z}}$, which in a certain region of spacetime (subject to the constraints imposed within the Poincare theorem) satisfies the equation $\mathbf{d} \widetilde{\mathbf{s}}-\widetilde{\mathbf{j}} \wedge \widetilde{\mathbf{s}}=\mathbf{0}$, can be presented in this region as $\widetilde{\mathbf{s}}=\mathbf{d} \widetilde{\mathbf{t}}-\widetilde{\mathbf{j}} \wedge \widetilde{\mathbf{t}}$, where $\widetilde{\mathbf{t}}$ is some $(m-1)$-form. There exists another theorem for $\mathbf{d}$, which can also be regarded as an analog of the Poincare theorem:

Any five-vector $m$-form $\widetilde{\mathbf{s}}$ with $m \geq 1$, which in a certain region of space-time (subject to the same constraints that are imposed within the Poincare theorem) satisfies the equation $\mathbf{d} \widetilde{\mathbf{s}}=\mathbf{0}$, can be presented in this region as a five-vector exterior derivative of some $(m-1)$-form. At $m=0$ from the above equation follows $\widetilde{\mathbf{s}}=\mathbf{0}$.

Proof: At $m \geq 1$, from equation (30) one has

$$
\mathbf{d} \widetilde{\mathbf{s}}=\mathbf{d} \widetilde{\mathbf{s}}+\widetilde{\mathbf{j}} \wedge \widetilde{\mathbf{s}}=\mathbf{d}\left(\widetilde{\mathbf{s}}^{\widetilde{\mathcal{Z}}}\right)+\mathbf{d}\left(\widetilde{\mathbf{s}}^{\widetilde{\mathcal{E}}}\right)+\widetilde{\mathbf{j}} \wedge\left(\widetilde{\mathbf{s}}^{\widetilde{\mathcal{Z}}}\right)=\mathbf{0} .
$$

Consequently, $\mathbf{d}\left(\widetilde{\mathbf{s}}^{\widetilde{\mathcal{Z}}}\right)=(\mathbf{d} \widetilde{\mathbf{s}})^{\widetilde{\mathcal{Z}}}=\mathbf{0}$, and according to the analog of the Poincare theorem for five-vector forms presented in the previous section, there exists an $(m-1)$-form $\widetilde{\mathbf{t}}$ such that $\widetilde{\mathbf{s}^{\mathcal{Z}}}=\mathbf{d}(\widetilde{\mathbf{t}} \widetilde{\mathcal{Z}})$. The $\widetilde{\mathcal{E}}$ component of equation (37) yields $\mathbf{d}(\widetilde{\mathbf{s}} \widetilde{\mathcal{E}})+\widetilde{\mathbf{j}} \wedge\left(\widetilde{\mathbf{s}}^{\widetilde{\mathcal{Z}}}\right)=$

$$
\begin{aligned}
& (\mathbf{d} \widetilde{\mathbf{s}})^{\widetilde{\mathcal{E}}}=\mathbf{0}, \text { so } \\
& \mathbf{d}(\widetilde{\mathbf{s}})=-\widetilde{\mathbf{J}} \wedge \widetilde{\mathbf{s}} \widetilde{\mathcal{Z}}=-\widetilde{\mathbf{j}} \wedge \mathbf{d}\left(\widetilde{\mathbf{t}} \widetilde{\mathcal{Z}}^{-}\right)=\mathbf{d}\left(\widetilde{\mathbf{j}} \wedge \widetilde{\mathbf{t}}^{\widetilde{\mathcal{Z}}}\right) .
\end{aligned}
$$

At $m \geq 2$, there exists an $(m-1)$-form $\widetilde{\mathbf{r}}$ such that $\widetilde{\mathbf{s}} \widetilde{\mathcal{E}}=\widetilde{\mathbf{j}} \wedge \widetilde{\mathbf{t}} \widetilde{\mathcal{Z}}+\mathbf{d}\left(\widetilde{\mathbf{r}}^{\mathcal{E}}\right)$, and since $\widetilde{\mathbf{j}} \wedge \widetilde{\mathbf{r}}^{\widetilde{\mathcal{E}}}=\mathbf{0}$, one obtains

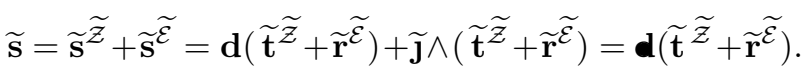


At $m=1, \widetilde{\mathbf{t}}$ is a scalar function, so $\widetilde{\mathbf{t}}^{\widetilde{\mathcal{Z}}}=\widetilde{\mathbf{t}} \equiv t$. From equation (38) it then follows that $\widetilde{\mathbf{s}} \widetilde{\mathcal{E}}-t \cdot \widetilde{\mathbf{j}}=$ const $\cdot \widetilde{\mathbf{J}}$, and since $\mathbf{d}$ (const) $=\mathbf{0}$, one obtains $\widetilde{\mathbf{s}}=\widetilde{\mathbf{s}} \widetilde{\widetilde{z}}+\widetilde{\mathbf{s}} \overline{\mathcal{E}}=\mathbf{d}(t+$ const $)+(t+$ const $) \cdot \widetilde{\mathbf{j}}=\mathbf{d}(t+$ const $)$. Finally, at $m=0$, one has $\widetilde{\mathbf{s}}=\widetilde{\mathbf{z}}$, and the $\widetilde{\mathcal{E}}$ component of equation $\mathbf{d} \widetilde{\mathbf{s}}=\mathbf{0}$ yields $\widetilde{\mathbf{s}}=\mathbf{0}$.

It is not difficult to see that the $(m-1)$-form whose five-vector exterior derivative equals $\widetilde{\mathbf{s}}$ is unique only at $m=1$. At $m \geq 2$ there exists an infinite number of such $(m-1)$-forms, but the difference of any two of them is a five-vector exterior derivative of some $(m-2)$-form.

\section{E. Five-vector flux and reflected five-vector exterior derivative}

The concept of exterior derivative is directly related to the concept of flux: by definition, for any fivevector form $\widetilde{\mathbf{s}}$, the exterior derivative $\mathbf{d} \widetilde{\mathbf{s}}$ is such that its integral over the interior of any limited volume of appropriate dimension equals the flux of $\widetilde{\mathbf{s}}$ through the volume boundary. We now have a generalization of $\mathbf{d}$ : the five-vector exterior derivative $\mathbf{d}$, and one can use the above scheme in the opposite direction to obtain the five-vector generalization of the flux, defining the latter as a quantity which for a given form $\widetilde{\mathbf{s}}$ and any limited volume $V$ of appropriate dimension, equals the integral of $\boldsymbol{d} \widetilde{\mathbf{s}}$ over $V$. Let us call this quantity the five-vector flux of form $\widetilde{\mathbf{s}}$ through volume $V$ and denote it as

$$
\oint_{V} \widetilde{\mathbf{s}}
$$

The formal analog of the generalized Stokes theorem for can then be presented as

$$
\int_{V} \mathbf{d} \widetilde{\mathbf{s}}=\oint_{V} \widetilde{\mathbf{s}}
$$

Let us now find the expression for the five-vector flux in the form of an integral of $\widetilde{\mathbf{s}}$. If $\widetilde{\mathbf{s}}$ is an $m$-form, then $\mathbf{d} \widetilde{\mathbf{s}}$ can be integrated over volumes of dimension $m+1$ and $m$. In the former case the integral will depend only on the $\widetilde{\mathcal{Z}}$-component of $\mathbf{d} \widetilde{\mathbf{s}}$, and since the latter coincides with $(\mathbf{d} \widetilde{\mathbf{s}})^{\widetilde{Z}}$, the flux corresponding to d will be exactly the same as the flux corresponding to $\mathbf{d}$.

The integral of $\boldsymbol{d} \widetilde{s}$ over an $m$-dimensional volume $V$ can be presented, by using equation (30) and the generalized Stokes theorem (15), as

$$
\begin{aligned}
\int_{V} \mathbf{d} \widetilde{\mathbf{s}}= & \int_{V} \mathbf{d} \widetilde{\mathbf{s}}+\int_{V} \widetilde{\mathbf{j}} \wedge \widetilde{\mathbf{s}} \\
& =\oint_{\partial V} \widetilde{\mathbf{s}}+(-1)^{m} \int_{V} \widetilde{\mathbf{s}} \wedge \widetilde{\mathbf{j}}
\end{aligned}
$$

One should now notice that the forms $\widetilde{\mathbf{s}}$ and $\widetilde{\mathbf{t}} \equiv \widetilde{\mathbf{s}} \wedge \widetilde{\mathbf{j}}$ satisfy equation (8) of section $B$, which means that the second integral in the right-hand side of equation (40) is simply the integral of $\widetilde{\mathbf{s}}$ over $V$. One thus obtains the following expression for the five-vector flux of $m$-form $\widetilde{\mathbf{s}}$ through the $m$-dimensional volume $V$ :

$$
\oint_{V} \widetilde{\mathbf{s}}=\oint_{\partial V} \widetilde{\mathbf{s}}+(-1)^{m} \int_{V} \widetilde{\mathbf{s}} .
$$

One should notice that unlike the fluxes of five-vector forms considered in section C, the five-vector flux (41) depends on both components of form $\widetilde{\mathbf{s}}$.

An important consequence of the generalized Stokes theorem is the formula for integration by parts. In the case of five-vector forms, this formula can be easily obtained by integrating both sides of equation (22a) over a given volume $V$ and then using equation (15) to convert the integral of $\mathbf{d}(\widetilde{\mathbf{s}} \wedge \widetilde{\mathbf{t}})$ over $V$ into the integral of $\widetilde{\mathbf{s}} \wedge \widetilde{\mathbf{t}}$ over $\partial V$. After rearranging the terms, one has

$$
\int_{V} \mathbf{d} \widetilde{\mathbf{s}} \wedge \widetilde{\mathbf{t}}=\oint_{\partial V} \widetilde{\mathbf{s}} \wedge \widetilde{\mathbf{t}}-(-1)^{m} \int_{V} \widetilde{\mathbf{s}} \wedge \mathbf{d} \widetilde{\mathbf{t}},
$$

where $m$ is the rank of $\widetilde{\mathbf{s}}$. The generalized Stokes theorem itself can be regarded as a particular case of this formula where $\widetilde{\mathbf{t}}$ is the constant unity 0 -form. Let us now derive the analog of this formula for $\mathbf{d}$

Following the same procedure, one integrates both sides of equation (34a) over a given volume $V$ and then uses equation (39) to convert the integral of $\mathbf{d}(\widetilde{\mathbf{s}} \wedge$ $\widetilde{\mathbf{t}})$ over $V$ into the five-vector flux of $\widetilde{\mathbf{s}} \wedge \widetilde{\mathbf{t}}$. After rearranging the terms, one obtains

$$
\begin{aligned}
\int_{V} \mathbf{d} \widetilde{\mathbf{s}} \wedge \widetilde{\mathbf{t}} & =\oint_{V} \widetilde{\mathbf{s}} \wedge \widetilde{\mathbf{t}} \\
& -(-1)^{m} \int_{V} \widetilde{\mathbf{s}} \wedge \mathbf{d} \widetilde{\mathbf{t}}+\int_{V} \widetilde{\mathbf{j}} \wedge \widetilde{\mathbf{s}} \wedge \widetilde{\mathbf{t}} .
\end{aligned}
$$

It is a simple matter to see that for volume $V$ with dimension one unit greater than the rank of $\widetilde{\mathbf{s}} \wedge \widetilde{\mathbf{t}}$, this equation reproduces formula (42). For volume $V$ with dimension equal to the rank of $\widetilde{\mathbf{s}} \wedge \widetilde{\mathbf{t}}$, it gives one a new formula, which, however, is not very useful since the first term in its right-hand side includes an integral over the interior of $V$ and, in addition, there exists a third term, which is also an integral over $V$. A more useful formula can be obtained if in the right-hand side of equation (43) one isolates the integral over the boundary of $V$ and combines everything else into a single second term. After simple transformations one obtains:

$$
\begin{aligned}
\int_{V} \mathbf{d} \widetilde{\mathbf{s}} \wedge \widetilde{\mathbf{t}} & =\oint_{\partial V} \widetilde{\mathbf{s}} \wedge \widetilde{\mathbf{t}} \\
& -(-1)^{m} \int_{V} \widetilde{\mathbf{s}} \wedge(d \widetilde{\mathbf{t}}-2 \widetilde{\mathbf{j}} \wedge \widetilde{\mathbf{t}}) .
\end{aligned}
$$


We thus see that it makes sense to consider one more operator, which will be denoted as $\mathbf{d}^{\star}$ and will be called the reflected five-vector exterior derivative. By definition, for any form $\widetilde{\mathbf{t}}$,

$$
\mathbf{d}^{\star} \widetilde{\mathbf{t}}=\mathbf{d} \widetilde{\mathbf{t}}-\widetilde{\mathbf{j}} \wedge \widetilde{\mathbf{t}}
$$

Formula (44) can now be rewritten as

$$
\int_{V} \mathbf{d} \widetilde{\mathbf{s}} \wedge \widetilde{\mathbf{t}}=\oint_{\partial V} \widetilde{\mathbf{s}} \wedge \widetilde{\mathbf{t}}-(-1)^{m} \int_{V} \widetilde{\mathbf{s}} \wedge \mathbf{d}^{\star} \widetilde{\mathbf{t}}
$$

It is easy to see that the latter equation can also be presented as

$$
\int_{V} \mathbf{d}^{\star} \widetilde{\mathbf{s}} \wedge \widetilde{\mathbf{t}}=\oint_{\partial V} \widetilde{\mathbf{s}} \wedge \widetilde{\mathbf{t}}-(-1)^{m} \int_{V} \widetilde{\mathbf{s}} \wedge \mathbf{d} \widetilde{\mathbf{t}} .
$$

Formulae (46) and (47) can be derived directly from the following equations:

$$
\begin{aligned}
\mathbf{d}(\widetilde{\mathbf{s}} \wedge \widetilde{\mathbf{t}}) & =\mathbf{d} \widetilde{\mathbf{s}} \wedge \widetilde{\mathbf{t}}+(-1)^{m} \widetilde{\mathbf{s}} \wedge \mathbf{d}^{\star} \widetilde{\mathbf{t}} \\
& =\mathbf{d}^{\star} \widetilde{\mathbf{s}} \wedge \widetilde{\mathbf{t}}+(-1)^{m} \widetilde{\mathbf{s}} \wedge \mathbf{d} \widetilde{\mathbf{t}}
\end{aligned}
$$

which are analogs of equations (22a) and (34a).

In order to express $\mathbf{d}^{\star} \widetilde{\mathbf{t}}$ in terms of its components, it is convenient to introduce the corresponding analog of the operator $\boldsymbol{\partial}_{\mathbf{u}}$, which I will denote as $\boldsymbol{\partial}_{\mathbf{u}}^{\star}$. By definition, for any scalar function $f$

$$
\partial_{\mathbf{u}}^{\star} f \equiv \partial_{\mathbf{u}} f-\varsigma \lambda_{\mathbf{u}} f
$$

From equation (28) one can then derive that

$$
\partial_{\mathbf{u}}(f g)=\boldsymbol{\partial}_{\mathbf{u}} f \cdot g+f \cdot \boldsymbol{\partial}_{\mathbf{u}}^{\star} g=\boldsymbol{\partial}_{\mathbf{u}}^{\star} f \cdot g+f \cdot \boldsymbol{\partial}_{\mathbf{u}} g,
$$

which is similar to equations (48) for $\mathbf{d}$, $\mathbf{d}$ and $\mathbf{d}^{\star}$. Using this new notation, one can present the reflected five-vector exterior derivative of $m$-form (16) as

$$
\mathbf{d}^{\star} \widetilde{\mathbf{t}}=\boldsymbol{\partial}_{A}^{\star} t_{\left|A_{1} \ldots A_{m}\right|} \widetilde{\mathbf{o}}^{A} \wedge \widetilde{\mathbf{o}}^{A_{1}} \wedge \ldots \wedge \widetilde{\mathbf{o}}^{A_{m}} .
$$

One can then derive the analogs of equations (31) through (34), which will differ from the latter in that everywhere $\mathbf{d}$ will be replaced with $\mathbf{d}^{\star}$ and $\boldsymbol{\partial}_{\mathbf{u}}$ will be replaced with $\partial_{\mathbf{u}}^{\star}$.

\section{F. Euler-Lagrange equations for classical scalar fields}

A good illustration to the ideas developed in this paper can be found in the Lagrange formalism for classical scalar fields. Let us suppose that we have $N$ such fields and let us denote them as $\phi_{\ell}(\ell$ runs 1 through $N$ and lables the fields, not components). For simplicity let us confine ourselves to the case where all $\phi_{\ell}$ are real. Mathematically, the action $S$ corresponding to these fields is an invariant four-dimensional integral of the type considered in section B. In local field theory, its integrand depends on the values of the fields and of their first derivatives at the integration point. Thus, for an arbitrary four-dimensional volume $V$ one has

$$
S(V)=\int_{V} \mathcal{L}
$$

where $\mathcal{L}=\mathcal{L}\left(\mathbf{d} \phi_{\ell}, \phi_{\ell}\right)$. According to section $\mathrm{B}$, the Lagrangian density $\mathcal{L}$ can be regarded as a fourvector 4 -form, or as a five-vector 4 -form, or as a fivevector 5 -form. Let us first recall the traditional interpretation. In this case

$$
\mathcal{L}=\frac{1}{4 !} \mathcal{L}_{\alpha \beta \gamma \delta} \mathbf{d} x^{\alpha} \wedge \mathbf{d} x^{\beta} \wedge \mathbf{d} x^{\gamma} \wedge \mathbf{d} x^{\delta},
$$

and for a given volume $V$,

$$
\int_{V} d^{4} x<\mathcal{L}, \mathbf{E}_{0} \wedge \mathbf{E}_{1} \wedge \mathbf{E}_{2} \wedge \mathbf{E}_{3}>=\int_{V} d^{4} x \mathcal{L}_{0123},
$$

where $\mathbf{E}_{\alpha}$ is the corresponding coordinate four-vector basis. The equations of motion for the fields $\phi_{\ell}$ are obtained from the action principle: the physical fields $\phi_{\ell}$ are such that their variation inside a given volume $V$ with the boundary condition $\left.\delta \phi_{\ell}\right|_{\partial V}=0$ yields a zero first variation of the action corresponding to $V$. In the standard way, from this principle one can derive the corresponding Euler-Lagrange equations:

$$
\partial_{\mu}\left[\frac{\partial \mathcal{L}_{0123}}{\partial\left(\partial_{\mu} \phi_{\ell}\right)}\right]=\frac{\partial \mathcal{L}_{0123}}{\partial \phi_{\ell}},
$$

which, equivalently, can be presented as

$$
\begin{aligned}
\frac{1}{4 !} \partial_{\mu} & \left\{\frac{\partial \mathcal{L}_{\alpha \beta \gamma \delta}}{\partial\left(\partial_{\mu} \phi \ell\right)}\right\} \mathbf{d} x^{\alpha} \wedge \mathbf{d} x^{\beta} \wedge \mathbf{d} x^{\gamma} \wedge \mathbf{d} x^{\delta} \\
= & \frac{1}{4 !}\left\{\frac{\partial \mathcal{L}_{\alpha \beta \gamma \delta}}{\partial \phi_{\ell}}\right\} \mathbf{d} x^{\alpha} \wedge \mathbf{d} x^{\beta} \wedge \mathbf{d} x^{\gamma} \wedge \mathbf{d} x^{\delta} .
\end{aligned}
$$

One should now recall the invariant definition of the derivatives of $\mathcal{L}$ with respect to $\phi_{\ell}$ and $\mathbf{d} \phi_{\ell}$ and find that

$$
\left[\frac{\partial \mathcal{L}}{\partial \phi_{\ell}}\right]_{\alpha \beta \gamma \delta}=\frac{\partial \mathcal{L}_{\alpha \beta \gamma \delta}}{\partial \phi_{\ell}},
$$

so the four-vector 4 -form in the right-hand side of equation (50) is exactly $\partial \mathcal{L} / \partial \phi_{\ell}$. In a similar manner one can find that the quantities in the curly brackets in the left-hand side of equation (50) are components of the four-vector-valued 4 -form $\partial \mathcal{L} / \partial\left(\mathbf{d} \phi_{\ell}\right)$. One can then use formula (63) of Appendix to present this side of equation (50) as

$$
\begin{aligned}
\partial_{\alpha} & \left\{\frac{\partial \mathcal{L}_{\mu|\beta \gamma \delta|}}{\partial\left(\partial_{\mu} \phi_{\ell}\right)}\right\} \mathbf{d} x^{\alpha} \wedge \mathbf{d} x^{\beta} \wedge \mathbf{d} x^{\gamma} \wedge \mathbf{d} x^{\delta} \\
& =\mathbf{d}\left\{\frac{\partial \mathcal{L}}{\partial\left(\mathbf{d} \phi_{\ell}\right)}\right\}_{\mu|\beta \gamma \delta|}^{\mu} \wedge \mathbf{d} x^{\beta} \wedge \mathbf{d} x^{\gamma} \wedge \mathbf{d} x^{\delta} \equiv \mathbf{d} \widetilde{\mathbf{J}}^{\ell},
\end{aligned}
$$


where $\widetilde{\mathbf{J}}^{\ell}$ is the scalar-valued 3 -form obtained from $\partial \mathcal{L} / \partial\left(\mathbf{d} \phi_{\ell}\right)$ by contracting its upper and its first lower four-vector indices. We thus see that the EulerLagrange equations (50) can be cast into the following abstract form:

$$
\mathbf{d} \widetilde{\mathbf{J}}^{\ell}=\widetilde{\mathbf{K}}^{\ell}
$$

where $\widetilde{\mathbf{K}}^{\ell} \equiv \partial \mathcal{L} / \partial \phi_{\ell}$. The integral formulation of this relation is the following: the flux of the fourvector 3 -form $\widetilde{\mathbf{J}}^{\ell}$ through the boundary of any limited four-dimensional volume $V$ equals the integral of the four-vector 4 -form $\widetilde{\mathbf{K}}^{\ell}$ over the interior of $V$.

Let us now turn to the other two possible interpretations of $\mathcal{L}$.

In the case where the Lagrangian density is regarded as a five-vector 4 -form one obtains practically the same results except that now in all the formulae all Greek indices are five-vector ones and everywhere $\mathcal{L}$ is replaced with $\mathcal{L}^{\widetilde{\mathcal{Z}}}$. Nothing is said about the $\widetilde{\mathcal{E}}$ component of $\mathcal{L}$ and unless some additional ideas are invokes, it has no relation to the Lagrange formalism.

More interesting results are obtained if $\mathcal{L}$ is regarded as a five-vector 5-form. In this case

$$
\mathcal{L}=\frac{1}{5 !} \mathcal{L}_{A B C D E} \widetilde{\mathbf{o}}^{A} \wedge \widetilde{\mathbf{o}}^{B} \wedge \widetilde{\mathbf{o}}^{C} \wedge \widetilde{\mathbf{o}}^{D} \wedge \widetilde{\mathbf{o}}^{E},
$$

and for a given volume $V$,

$\int_{V} d^{4} x<\mathcal{L}, \mathbf{e}_{0} \wedge \mathbf{e}_{1} \wedge \mathbf{e}_{2} \wedge \mathbf{e}_{3} \wedge \mathbf{e}_{5}>=\int_{V} d^{4} x \mathcal{L}_{01235}$

where $\mathbf{e}_{A}$ is the corresponding passive regular coordinate basis. Following the standard procedure, one can obtain the Euler-Lagrange equations

$$
\partial_{\mu}\left[\frac{\partial \mathcal{L}_{01235}}{\partial\left(\partial_{\mu} \phi_{\ell}\right)}\right]-\frac{\partial \mathcal{L}_{01235}}{\partial \phi_{\ell}}=0,
$$

and considering that in a passive regular basis one has $\boldsymbol{\partial}_{\mu}=\partial_{\mu}$ and $\boldsymbol{\partial}_{5}=1$, one can rewrite them as

$$
\boldsymbol{\partial}_{\mu}\left[\frac{\partial \mathcal{L}_{01235}}{\partial\left(\boldsymbol{\partial}_{\mu} \phi_{\ell}\right)}\right]-\boldsymbol{\partial}_{5}\left[\frac{\partial \mathcal{L}_{01235}}{\partial\left(\boldsymbol{\partial}_{5} \phi_{\ell}\right)}\right]=0
$$

One should then change the sign of the second term by replacing one of the $\boldsymbol{\partial}_{A}$ with $\boldsymbol{\partial}_{A}^{\star}$. This enables one to present equation (52) as

$$
\frac{1}{5 !} \boldsymbol{\partial}_{H}\left\{\frac{\partial \mathcal{L}_{A B C D E}}{\partial\left(\boldsymbol{\partial}_{H}^{\star} \phi_{\ell}\right)}\right\} \widetilde{\mathbf{o}}^{A} \wedge \widetilde{\mathbf{o}}^{B} \wedge \widetilde{\mathbf{o}}^{C} \wedge \widetilde{\mathbf{o}}^{D} \wedge \widetilde{\mathbf{o}}^{E}=\mathbf{0}(53 \mathrm{a})
$$

or as

$$
\frac{1}{5 !} \boldsymbol{\partial}_{H}^{\star}\left\{\frac{\partial \mathcal{L}_{A B C D E}}{\partial\left(\boldsymbol{\partial}_{H} \phi_{\ell}\right)}\right\} \widetilde{\mathbf{o}}^{A} \wedge \widetilde{\mathbf{o}}^{B} \wedge \widetilde{\mathbf{o}}^{C} \wedge \widetilde{\mathbf{o}}^{D} \wedge \widetilde{\mathbf{o}}^{E}=\mathbf{0} .
$$

One should now recall the invariant definition of the derivatives of $\mathcal{L}$ and show that the quantities in the curly brackets in equations (53a) and (53b) are exactly the components of the derivatives $\partial \mathcal{L} / \partial\left(\mathbf{d}^{\star} \phi_{\ell}\right)$ and $\partial \mathcal{L} / \partial\left(\mathbf{d} \phi_{\ell}\right)$, respectively. Finally, one can use formula (64) of Appendix to present equation (53a) as

$\mathbf{d}\left\{\frac{\partial \mathcal{L}}{\partial\left(\mathbf{d}^{\star} \phi_{\ell}\right)}\right\}_{H|A B C D|}^{H} \wedge \widetilde{\mathbf{o}}^{A} \wedge \widetilde{\mathbf{o}}^{B} \wedge \widetilde{\mathbf{o}}^{C} \wedge \widetilde{\mathbf{o}}^{D}=\mathbf{0}(54 \mathrm{a})$

and equation (53b) as

$\mathbf{d}^{\star}\left\{\frac{\partial \mathcal{L}}{\partial\left(\mathbf{d} \phi_{\ell}\right)}\right\}_{H|A B C D|}^{H} \wedge \widetilde{\mathbf{o}}^{A} \wedge \widetilde{\mathbf{o}}^{B} \wedge \widetilde{\mathbf{o}}^{C} \wedge \widetilde{\mathbf{o}}^{D}=\mathbf{0}$,

and it is a simple matter to show that equation (54b) can be obtained from equation (54a) by changing the sign of the $\widetilde{\mathcal{Z}}$-components of all the quantities involved. We thus see that the Euler-Lagrange equations in this case can be presented as

$$
\mathbf{d} \mathbf{\Lambda}^{\ell}=\mathbf{0}
$$

where $\boldsymbol{\Lambda}^{\ell}$ is the scalar-valued five-vector 4 -form obtained from $\partial \mathcal{L} / \partial\left(\boldsymbol{d}^{\star} \phi_{\ell}\right)$ by contracting its upper and its first lower five-vector indices:

$$
\left(\boldsymbol{\Lambda}^{\ell}\right)_{A B C D}=\left[\partial \mathcal{L} / \partial\left(\mathbf{d}^{\star} \phi_{\ell}\right)\right]_{H A B C D}^{H} .
$$

In the language of integrals, equation (55) means that the five-vector flux of the 4 -form $\boldsymbol{\Lambda}^{\ell}$ through any limited four-dimensional volume $V$ is zero:

$$
\oint_{V} \Lambda^{\ell}=0
$$

\section{G. Five-vector Levi-Civita tensor and dual forms}

As on any other vector space endowed with a nondegenerate inner product, one can define on $V_{5}$ a completely antisymmetric tensor, $\boldsymbol{\epsilon}$, whose rank in this case is five. The magnitude of its only independent component, say, of $\epsilon_{01235}$, is fixed by the condition:

$$
\begin{aligned}
& \epsilon_{01235}=+1 \text { in any orthonormal } \\
& \text { basis with positive orientation }{ }^{2},
\end{aligned}
$$

and then the components of $\boldsymbol{\epsilon}$ in an arbitrary fivevector basis $\mathbf{e}_{A}$ will be

$$
\epsilon_{A B C D E}=\eta \cdot|h|^{1 / 2} \cdot|A B C D E|,
$$

where $h$ denotes the determinant of the matrix $h_{A B} \equiv$ $h\left(\mathbf{e}_{A}, \mathbf{e}_{B}\right)$ (at some particular choice of the constant

\footnotetext{
${ }^{2}$ The notion of orientation for five-vector bases is defined in the usual way. In the following I will consider positive the orientation of a normalized regular basis for which the associated four-vector basis has positive four-dimensional orientation.
} 
$\xi)$; parameter $\eta$ equals +1 if the basis $\mathbf{e}_{A}$ has positive orientation and -1 otherwise; and the symbol $|A B C D E|$ is defined in the usual way:

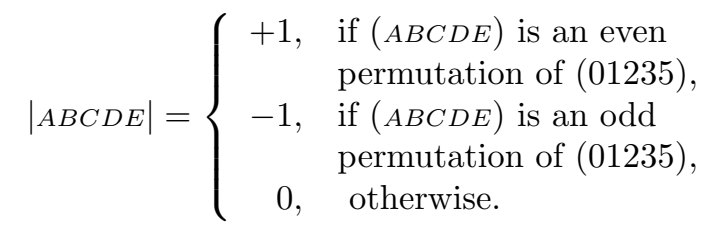

It is easy to show that in any standard basis one has $h=h_{55} \cdot g$, where $g$ denotes the determinant of the $4 \times 4$ matrix $g_{\alpha \beta} \equiv g\left(\mathbf{e}_{\alpha}, \mathbf{e}_{\beta}\right)$, and therefore in any active regular basis one has

$$
\epsilon_{01235}=\eta \cdot|g|^{1 / 2} \cdot \kappa,
$$

where $\kappa \equiv|\xi|^{1 / 2}$, and in any passive regular basis one has

$$
\epsilon_{01235}=\eta \cdot|g|^{1 / 2} \cdot \varpi,
$$

where $\varpi \equiv|\xi|^{1 / 2} \cdot \varsigma^{-1}$ and $\varsigma$ is the dimensional constant introduced in section 3 of part II.

As in the case of the four-vector Levi-Civita tensor, it is convenient to introduce the completely contravariant tensor corresponding to $\boldsymbol{\epsilon}$, whose components are

$$
\epsilon^{A B C D E}=h^{A A^{\prime}} h^{B B^{\prime}} h^{C C^{\prime}} h^{D D^{\prime}} h^{E E^{\prime}} \epsilon_{A^{\prime} B^{\prime} C^{\prime} D^{\prime} E^{\prime}},
$$

where matrix $h^{A B}$ is the inverse of $h_{A B}$. It is not difficult to demonstrate that in any standard basis

$$
\epsilon^{A B C D E}=-\operatorname{sign} \xi \cdot \eta \cdot|h|^{-1 / 2} \cdot|A B C D E|,
$$

and therefore

$$
\epsilon^{A B C D E} \epsilon_{A B C D E}=-5 ! \cdot \operatorname{sign} \xi .
$$

The latter equation is a particular case of the general relation that expresses the contraction of $\boldsymbol{\epsilon}$ with its completely contravariant counterpart over a certain number of indices, in terms of the so-called permutation tensors:

$$
\begin{aligned}
\epsilon^{A_{1} \ldots A_{m} C_{1} \ldots C_{5-m}} \epsilon_{B_{1} \ldots B_{m} C_{1} \ldots C_{5-m}} & \\
= & -(5-m) ! \cdot \operatorname{sign} \xi \cdot \delta^{A_{1} A_{2} \ldots A_{m}} B_{1} B_{2} \ldots B_{m}
\end{aligned}
$$

where $m$ can be any integer from 0 to 5 and

$$
\delta_{B_{1} B_{2} \ldots B_{m}}^{A_{1} A_{2} \ldots A_{m}} \equiv m ! \delta_{B_{1}}^{\left[A_{1}\right.} \delta_{B_{2}}^{A_{2}} \ldots \delta_{B_{m}}^{\left.A_{m}\right]} .
$$

Let me say a few words about the differential properties of $\boldsymbol{\epsilon}$. Since the normalization of the latter is determined by the inner product $h$ and since any nondegenerate $h$ is not conserved by parallel transport, it is a priori not clear whether or not $\boldsymbol{\epsilon}$ is a covariantly constant tensor. One can gain an understanding of the situation from the following general reasoning.

Let us consider an arbitrary standard orthonormal basis $\mathbf{e}_{A}$ at some space-time point $Q$ and let us parallel transport it to a neighbouring point $Q^{\prime}$ along some continuous curve $\mathcal{C}$ connecting $Q$ with $Q^{\prime}$. Let us denote the transported vector $\mathbf{e}_{A}$ as $\mathbf{e}^{\prime}{ }_{A}$.

Owing to the general properties of five-vector parallel transport discussed in section 3 of part II, $\mathbf{e}^{\prime}{ }_{5}$ is, again, a vector from $\mathcal{E}$ and the $\mathcal{Z}$-components of $\mathbf{e}^{\prime}{ }_{\alpha}$ are orthogonal one to another and are normalized. Therefore,

$$
\begin{aligned}
\boldsymbol{\epsilon}\left(\mathbf{e}_{0}^{\prime}, \mathbf{e}^{\prime}{ }_{1}, \mathbf{e}^{\prime}{ }_{2},\right. & \left.\mathbf{e}^{\prime}{ }_{3}, \mathbf{e}^{\prime}{ }_{5}\right) \\
& =\boldsymbol{\epsilon}\left(\mathbf{e}_{0}^{\prime \mathcal{Z}}, \mathbf{e}_{1}^{\prime \mathcal{Z}}, \mathbf{e}_{2}^{\prime \mathcal{Z}}, \mathbf{e}^{\prime \mathcal{Z}}, \mathbf{e}^{\prime}{ }_{5}\right) \\
& =\left(\lambda_{\mathbf{e}^{\prime}{ }_{5}} / \lambda_{\mathbf{e}_{5}}\right) \boldsymbol{\epsilon}\left(\mathbf{e}_{0}, \mathbf{e}_{1}, \mathbf{e}_{2}, \mathbf{e}_{3}, \mathbf{e}_{5}\right),
\end{aligned}
$$

and so $\boldsymbol{\epsilon}$ is covariantly constant or not depending on whether or not parallel transport conserves the length of the vectors from $\mathcal{E}$. The same result can be obtained by computing the covariant derivative of $\boldsymbol{\epsilon}$ in components and finding that in any standard fivevector basis where the length of the fifth basis vector is constant

$$
\epsilon_{A B C D E ; \mu}=-G_{5 \mu}^{5} \epsilon_{A B C D E} .
$$

Thus, in the case where the connection for five-vectors possesses the local symmetry described in section 3 of part II, tensor $\boldsymbol{\epsilon}$ is covariantly constant.

As its four-vector analog, tensor $\boldsymbol{\epsilon}$ can be used for converting multivectors into forms and vice versa. Namely, if $\mathbf{w}$ is a multivector on $V_{5}$ of rank $m$ $(0 \leq m \leq 5)$ with components $w^{A_{1} \ldots A_{m}}$, one can construct from it a five-vector $(5-m)$-form with the components

$$
(m !)^{-1} w^{B_{1} \ldots B_{m}} \epsilon_{B_{1} \ldots B_{m} A_{1} \ldots A_{5-m}} .
$$

To formulate this correspondence in invariant form, one should regard $\boldsymbol{\epsilon}$ as an inner product defined for any two multivectors on $V_{5}$ whose ranks total up to 5 , assuming that

$$
\begin{aligned}
\boldsymbol{\epsilon}\left(\mathbf{e}_{A_{1}} \wedge \ldots \wedge\right. & \left.\mathbf{e}_{A_{m}}, \mathbf{e}_{B_{1}} \wedge \ldots \wedge \mathbf{e}_{B_{5-m}}\right) \\
& \equiv \boldsymbol{\epsilon}\left(\mathbf{e}_{A_{1}}, \ldots, \mathbf{e}_{A_{m}}, \mathbf{e}_{B_{1}}, \ldots, \mathbf{e}_{B_{5-m}}\right) .
\end{aligned}
$$

Regarding multivectors of rank $m$ as elements of a vector space with dimension $5 ! / m !(5-m)$ ! and identifying the linear forms on this space with five-vector $m$-forms, one can employ the same method that has been used in subsection 3.E of part II to define the maps $\vartheta_{g}$ and $\vartheta_{h}$ and put into correspondence to each multivector $\mathbf{w}$ of rank $m$ a certain form $\vartheta_{\epsilon}(\mathbf{w})$ of rank $5-m$ such that

$$
<\vartheta_{\epsilon}(\mathbf{w}), \mathbf{v}>=\boldsymbol{\epsilon}(\mathbf{w}, \mathbf{v})
$$


for any multivector $\mathbf{v}$ of rank $5-m$. It is a simple matter to check that the relation between the components of $\mathbf{w}$ and those of the form $\vartheta_{\epsilon}(\mathbf{w})$ is indeed given by formula (59).

There exists another correspondence between multivectors and forms, which is determined by the nondegenerate inner product $h$ on $V_{5}$ or, more precisely, by the inner product of multivectors induced by $h$. In this case to any multivector $\mathbf{w}$ of rank $m$ with components $w^{A_{1} \ldots A_{m}}$ one puts into correspondence a form of same rank with the components

$$
w^{B_{1} \ldots B_{m}} h_{B_{1} A_{1}} \ldots h_{B_{m} A_{m}} .
$$

This latter form, which I will denote as $\vartheta_{h}(\mathbf{w})$, can be defined invariantly by requiring that for any mulrivector $\mathbf{v}$ of rank $m$

$$
<\vartheta_{h}(\mathbf{w}), \mathbf{v}>=h(\mathbf{w}, \mathbf{v})
$$

where $h$ in this case denotes the inner product of multivectors of rank $m$ induced by the nondegenerate inner product on $V_{5}$.

One can now combine the maps $\vartheta_{\epsilon}$ and $\vartheta_{h}$ and define a one-to-one correspondence between forms or multivectors of rank $m$ and forms or multivectors of rank $5-m$. The image of an $m$-form $\widetilde{\mathbf{w}}$ with respect to this map will be called a form dual to $\widetilde{\mathbf{w}}$ and will be denoted as $\widetilde{\mathbf{w}}^{\text {dual }}$. It is evident that for any form $\widetilde{\mathbf{W}}$

$$
\widetilde{\mathbf{w}}^{\text {dual }}=\vartheta_{\epsilon} \circ \vartheta_{h}^{-1}(\widetilde{\mathbf{w}})
$$

and the components of $\widetilde{\mathbf{w}}^{\text {dual }}$ are expressed in terms of those of $\widetilde{\mathbf{w}}$ according to the well-known formula:

$$
\begin{aligned}
\left(\widetilde{\mathbf{w}}^{\text {dual }}\right)_{A_{1} \ldots A_{5-m}}=(m !)^{-1} w_{C_{1} \ldots C_{m}} \\
\quad \times h^{C_{1} B_{1}} \ldots h^{C_{m} B_{m}} \epsilon_{B_{1} \ldots B_{m} A_{1} \ldots A_{5-m}}
\end{aligned}
$$

It is easy to prove that

$$
\left(\widetilde{\mathbf{w}}^{\text {dual }}\right)^{\text {dual }}=-\operatorname{sign} \xi \cdot \widetilde{\mathbf{w}}
$$

and that the duality operation transforms the $\widetilde{\mathcal{Z}}$ and $\widetilde{\mathcal{E}}$-components of any form $\widetilde{\mathbf{w}}$ respectively into the $\widetilde{\mathcal{E}}$ - and $\widetilde{\mathcal{Z}}$-components of $\widetilde{\mathbf{w}}^{\text {dual }}$. Let me also note that in the case of five-vector forms, as in the case of forms associated with any other vector space of odd dimension, the rank of the dual form never equals that of the initial form, so in the general case one is not able to define the operation of dual rotation. The only exception are the 2-forms with the zero $\widetilde{\mathcal{E}}$-component, which the duality operation transforms into 3 -forms with the zero $\widetilde{\mathcal{Z}}$-component. Since there exists another correspondence between these two types of forms, given by equation (8) at $m=2$, one is able to define a map similar to the duality operation, which transforms a 2-form with the zero $\widetilde{\mathcal{E}}$-component into a 2 -form of the same type. It is easy to see that such an operation corresponds to the duality transformation of four-vector 2-forms.

In conclusion, let me mention one useful identity that involves dual forms: if $\widetilde{\mathbf{s}}$ and $\widetilde{\mathbf{t}}$ are any two fivevector forms of same rank, then

$$
\widetilde{\mathbf{s}} \wedge \widetilde{\mathbf{t}}^{\text {dual }}=\widetilde{\mathbf{s}}^{\text {dual }} \wedge \widetilde{\mathbf{t}}=h(\widetilde{\mathbf{s}}, \widetilde{\mathbf{t}}) \cdot \boldsymbol{\epsilon},
$$

where the inner product of forms $\widetilde{\mathbf{s}}$ and $\widetilde{\mathbf{t}}$ is defined in the usual way:

$$
h(\widetilde{\mathbf{s}}, \widetilde{\mathbf{t}})=s_{\left|A_{1} \ldots A_{m}\right|} t^{A_{1} \ldots A_{m}} .
$$

\section{Appendix: Index transposition identity}

There exists a very useful identity of purely combinatorial nature, which enables one to transpose a single index with a group of antisymmetrized indices if the number of the latter equals the number of values the indices run through. In the general case, this identity can be formulated as follows:

If the array $S_{i j_{1} \ldots j_{m}}(m \geq 2)$ is completely antisymmetric in $j_{1}, \ldots, j_{m}$ and all indices run through the same $m$ values, then

$$
S_{i j_{1} \ldots j_{m}}=m(-1)^{m+1} S_{\left[j_{1} \ldots j_{m}\right] i} .
$$

Proof : One has

$$
\begin{aligned}
S_{i j_{1} \ldots j_{m}}= & S_{i\left[j_{1} \ldots j_{m}\right]} \\
= & m S_{\left[i j_{1} \ldots j_{m}\right]}+S_{\left[j_{1}|i| j_{2} \ldots j_{m}\right]} \\
& -\ldots+(-1)^{m+1} S_{\left[j_{1} \ldots j_{m}\right] i},
\end{aligned}
$$

where, as usual, the notation $\left[j_{1} \ldots j_{k}|i| j_{k+1} \ldots j_{m}\right]$ means antisymmetrization with respect to all the indices inside the square brackets except for $i$. Since all indices run through $m$ values only, the first term in the right-hand side of equation (62) is identically zero. Since $S_{i j_{1} \ldots j_{m}}$ is antisymmetric in its last $m$ indices, one has

$$
\begin{array}{r}
(-1)^{k+1} S_{\left[j_{1} \ldots j_{k}|i| j_{k+1} \ldots j_{m}\right]} \\
=(-1)^{k+1}(-1)^{m-k} S_{\left[j_{1} \ldots j_{m}|i|\right]} \\
=(-1)^{m+1} S_{\left[j_{1} \ldots j_{m}\right] i}
\end{array}
$$

for all $k$ from 1 to $m-1$, so the remaining $m$ terms in the right-hand side of equation (62) are all equal, and equation (62) acquires the form of equation (61).

We will need the following two particular cases of identity (61): 
- If $S_{\alpha \beta \gamma \delta}^{\mu}=S_{[\alpha \beta \gamma \delta]}^{\mu}$ are components of a fourvector-valued four-vector 4 -form, then

$$
\frac{1}{4 !} \partial_{\mu} S_{\alpha \beta \gamma \delta}^{\mu}=\frac{1}{3 !} \partial_{[\alpha} T_{\beta \gamma \delta]},
$$

where $T_{\beta \gamma \delta} \equiv S_{\mu \beta \gamma \delta}^{\mu}$.

- If $S_{A B C D E}^{H}=S_{[A B C D E]}^{H}$ are components of a five-vector-valued five-vector 5 -form, then

$$
\frac{1}{5 !} \partial_{H} S_{A B C D E}^{H}=\frac{1}{4 !} \partial_{[A} T_{B C D E]} \cdot
$$

where $T_{B C D E} \equiv S_{H B C D E}^{H}$.

\section{Reference}

1. L.Schwartz, Analyse Mathématique, vol.II, Hermann, 1967. 


\title{
Computer Simulation Center in Internet
}

\author{
G.A. Tarnavsky \\ Institute of Computational Mathematics \\ and Mathematical Geophysics, Siberian Branch \\ of the Russian Academy of Sciences \\ Lavrentyev Avenue 6 \\ 630090, Novosibirsk, Russia \\ Email: Gennady.Tarnavsky@gmail.com \\ E.V. Vorozhtsov \\ Khristianovich Institute of Theoretical \\ and Applied Mechanics, Siberian Branch \\ of the Russian Academy of Sciences, \\ Institutskaya street $4 / 1$ \\ 630090, Novosibirsk, Russia \\ Email: vorozh@itam.nsc.ru
}

Abstract: The general description of infrastructure and content of SciShop.ru computer simulation center is given. This resource is a new form of knowledge generation and remote education using modern Cloud Computing technologies.

Keywords: Cloud computing, internet, information technologies, computer simulation, program complexes, remote access, remote education.

\section{Introduction}

Recent achievements in the area of information technologies and Internet give the reasons to believe that the new methods of the organization of the scientific knowledge exchange process have already been formed by now, and in the near future, one may expect a general passage from the conventional techniques of the scientific knowledge dissemination via the paper journals to their electronic counterparts.

The present paper is devoted to one of the advanced aspects of the scientific knowledge dissemination - a new form of the transfer of the developed intellectual product, the program complexes for solving various scientific and applied problems from the developers to the users.

The process of the transfer of computational complexes conventionally consisted of the fact that the consumer (the future user) acquired the codes (in some cases also the program texts) from the code developers and then installed them on his computer. To ensure a reliable and declared work of the program complex the user must have a similar operation medium, including the systems for visualizing the digital data.

The stage of the installation of computer programs is, on the whole, very complex even when a support by the developer is provided, and it often causes many difficulties the surmounting of which requires considerable, intrinsically non-productive expenses of intellectual efforts and time.

The new form of using the computational complexes frees from all problems related to the installation of the acquired program product.

A special site - the Computer Simulation Center - is created in the Internet. This Center hosts the program complexes with all their attributes: the preprocessor system for preparing the tasks, the processor system for executing the tasks (executing the computational operations), and the postprocessor system for the output of the obtained information in the digital and graphical forms.

The user must only formulate his own computational task by performing the input of numerical data and starting its numerical solution. He will be provided with the solution of his task upon the 


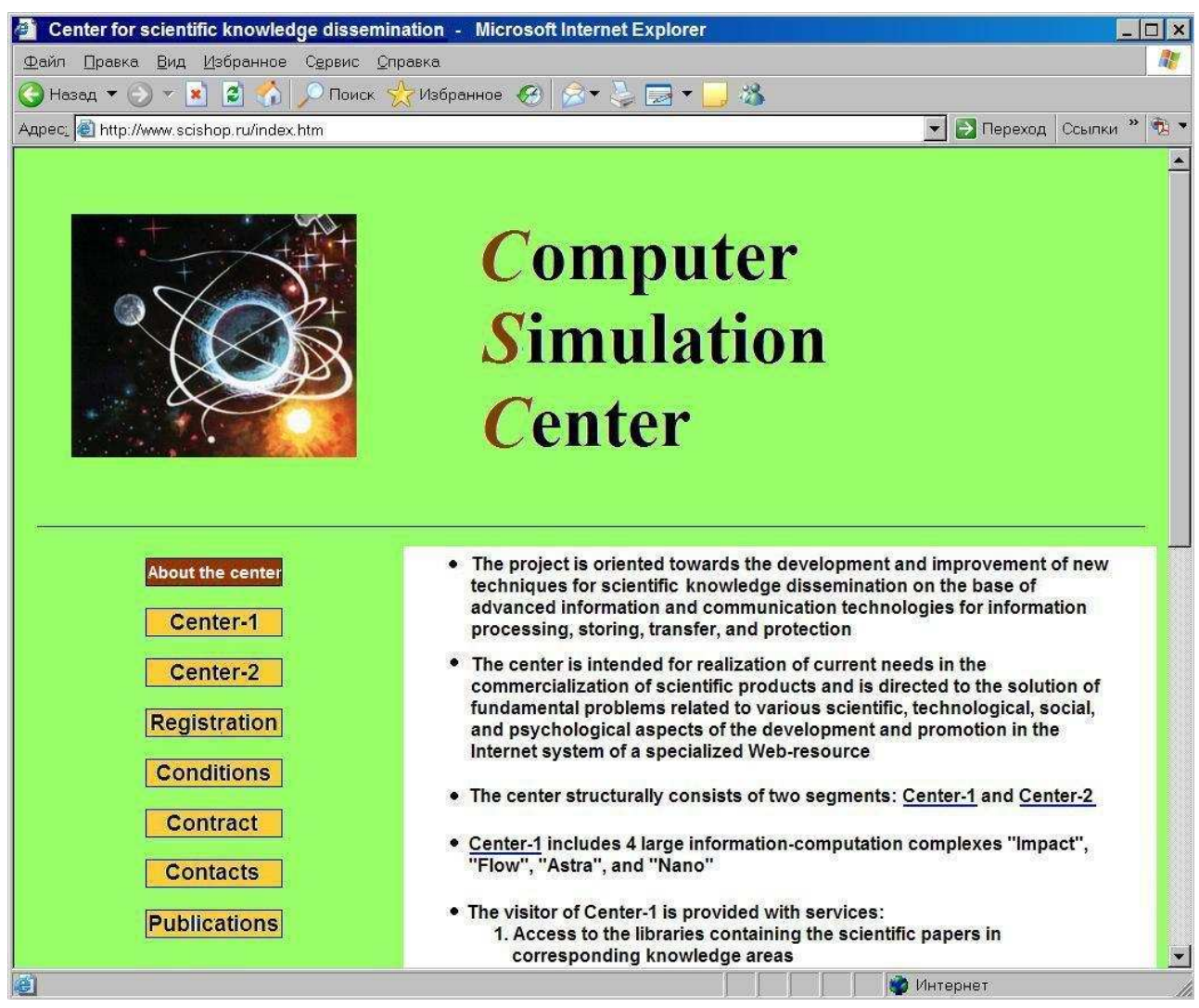

Figure 1: Main page of the Computer Simulation Center in Internet (a fragment).

termination of computations. Special services of the client's support must provide a comfort of the visitor's stay at the Center. In the economic terms, such a method of using the program complexes means their leasing from the resource developers.

The Computer Simulation Center SciShop.ru is a pioneer of this new direction of the development of the advanced information technologies. The Center was created as a result of the execution of the series of works (see, for example, [1, 2, 3, 4, 5, 6, 7, 8, 9, 10, 11]).

A general description and the infrastructure of the Center are presented in [1, 2, 3, and its content, the information-computational complexes for solving the tasks from a number of scientific areas are presented in 4, 5, 6, 7, 8, . At present, the Computer Simulation Center is functioning successfully in the Worldwide web and is subject to its continuous modernization and development (see [10, 11).

This project is directed to the application of breakthrough technologies in the domain of the development and improvement of new techniques for scientific knowledge dissemination with the use of the Worldwide web and is intended for a remote teaching of the specialists, postgraduates, and students to the methods of mathematical modelling and practical training in the solution of scientific and applied problems. The teaching and training are carried out on the basis of efficiently functioning program complexes whose composition is continuously enriched. 


\section{Computer Simulation Center SciShop.ru}

The project on the development of the Computer Simulation Center (http://www. SciShop.ru) is oriented towards the development and improvement of new techniques for scientific knowledge dissemination on the basis of the advanced info-communication technologies for the information processing, storing, transfer, and protection.

- The Center is intended for the realization of current needs in the commercialization of scientific products and is directed to the solution of fundamental problems related to various scientific, technological, social and psychological aspects of the development and promotion in the system of the specialized Internet Web resource.

- The Center structurally consists of two segments: Center-1 and Center-2.

- Center-1 includes four large information-computational complexes "Shock", "Flow", "Astra", and "Nano".

- The following services are provided for the visitor of Center-1:

1. The access to the libraries containing the scientific articles in corresponding knowledge areas.

2. The access to tabular and/or graphical databases containing the results of computer simulation of the corresponding computer tasks.

3. The access to processor systems enabling for the client himself the organization and execution of computer simulation of the problems, which are of interest to him.

4. The access to the locks to pass to the sites of the leading Russian and foreign journals in corresponding knowledge areas.

5. The access to the segment "Forum" for obtaining the consultations and discussion of problems.

- In the non-commercial regime, the visitor has the access to the demo versions of the systems of Center-1. For a full-scale access, one should register and perform the payment.

- The system of the Center for accepting the payments accepts the payment from any electronic payment systems (WebMoney, Yandex.Money, E-gold, etc., which enter the Robokassa consortium). The system for accepting payment with the use of the bank credit cards and SMS messages of the cellular communications has been developed and is now under verification.

- Center-2 is intended for positioning the program developments in various branches of knowledge without any limitations for the themes. The content of this section may be augmented by the resources of any specialists having the copyright for these information resources.

- All the specialists in the field of computer simulation in any knowledge areas, which have the program developments and wish to promote them, also on the onerous basis, are invited for a cooperation with Center-2. The specialists wishing to place their scientific products at Center-2 should familiarize themselves with the conditions of their placement, get in touch with the administration of the Center, and to sign a Contract.

- One can familiarize himself in detail with all aspects of the Center functioning in the special section "Publications" by using the corresponding hyperlink on the site Main page (Fig. 1).

\section{General characteristic of the arrangement of computations at the center and the advantages of the direct computer simulation in Internet}

The comfort of the client's stay in the Internet center and a convenient form of using its segments: the bibliographic section, databases containing already obtained tabular and graphical information, and especially the processor systems are the most important attributes of any program complex. Right this is usually most difficult for the user. 


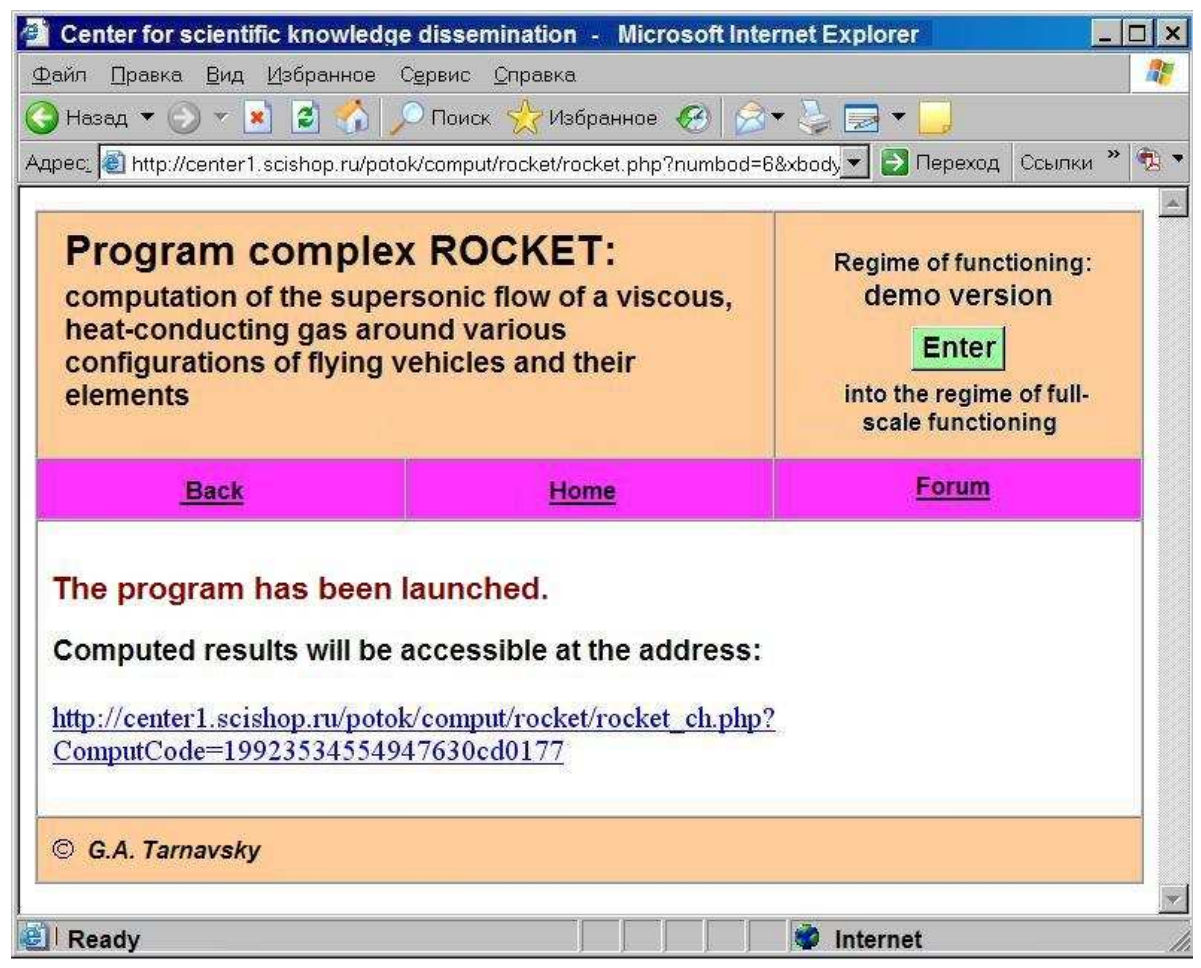

Figure 2: Site page with the messages for the client that the computation of the task formed by him has been launched, and its solution will be accessible at the indicated URL address.

The systems for the pre-processor preparation of tasks (the input of parameters and the start-up of processor systems) are arranged in a clear, convenient, and simplest form, which eliminates an ambiguous interpretation and difficulties for the specialists having even a small experience.

The operations with the processor systems are carried out by the user in the regime of remote access in the Worldwide web on its local portal - directly at the computer simulation center rather than on its own computer after the installation of the complex. This gives for the visitor of the Center a possibility to carry out the study of the computational complex, to organize the solution of the problem of interest, and to obtain the results of computer studies.

The transfer of a computational complex usually consists of the purchase of the license, documentation, and computer codes. After that, the user performs the installation of the purchased product on its own computer facility. As a rule, this occurs with large difficulties, which may be due to various reasons, from using different versions of the operating system to the peculiarities of the supporting systems installed on computers of the seller and the purchaser.

There are no such problems when the processor systems are placed at the Center. All the interfaces have been debugged, well tested and adjusted. The functioning of complexes is faultless in the specified ranges of parameters variation (note that one can always ask any question on the site forum and obtain the explanation). The fact that the user is freed from the necessity of buying the hardware (which is often very costly) necessary for performing the needed computations is one more advantage of this approach.The user in fact "leases" the hardware from the site developers only for the time of the solution of his task.

We emphasize that such an efficient method of using the processor complexes by the visitors of the Center has required the development and implementation of original solutions. Since neither of 
the Internet providers will permit the execution of many and, possibly, long-term computations on his node, which would take many resources and reduce the capacity of channels, one would need the use of a different scheme for execution of computations.

The client organizes at the Center his computational task (chooses the processor system and inputs the parameters in it) and starts up the computation on the system. The systems of this site segment on the support of tasks pack the task into a file and send it via the net to the computing center containing a number of computers, including the multi-processor systems. The task is solved here, the results (Fig. 2) are forwarded either to the Center, if the client waits for them, or to its home address in the net. This scheme has shown itself very well during its beta testing.

\section{The system for registering clients and commercial system of the center}

The program complexes of the Center may be used both free of charge (the demo versions) and on a commercial basis. The access to the regime of the full-scale functioning of the center is realized after the visitor registration in the specialized "book of record" (Fig. 3) and after the visitor transfers the user charge via the electronic payment systems.

The use of the specialized bank system "Robokassa" has been organized (together with the development of necessary interfaces) for the commercial segment of the center. This system enables the use of more than 20 electronic payment systems (WebMoney, Yandex.Money, Internet.Money, Internet.Groshi) as well as a number of foreign electronic payment systems of the type E-Gold, PayPal, MoneyBookers, EuroCash, etc. for the purpose of a significant broadening of the scope of clients. A system has also been implemented, which makes it possible to carry out the payments by using the SMS cellular communications (Fig. 4). The safety of the passage of payments, the transparency of their routing, the necessary messages for the client, the currency conversion in different electronic payment systems are guaranteed by special structures of the "Robokassa" system and have been checked in the course of the beta testing of the Computer simulation center.

One should, however, emphasize that the profit earning is not set at present as a predominant goal of the present many-sided project, which is a functional study of the problems of the scientific knowledge dissemination based on advanced information technologies.

\section{The center content}

The site SciShop.ru is a developing Center of computer simulation. At present, it contains four working information-computation complexes (ICCs):

- "Shock", the high-velocity internal aerodynamics: the computation of shock-wave structures at the inlet to the diffuser of a hypersonic scramjet engine;

- "Flow", the high-velocity external aerodynamics: the computation of the flow around the objects in the atmospheres of the Earth and Mars;

- "Astra", the computational astrophysics: simulation of the dynamics of processes in the intergalactic gas and protoplanet clouds;

- "Nano", microelectronics: computer support of the design of nano-structured semiconductor materials.

Each of these complexes includes the bibliographic section, tabular and graphical databases, which contain the results of the computation of problems in their subject areas as well as the processor systems, which enable the visitor to organize independently the solution of the task of interest. All the resources were created and improved in the course of the execution of numerous computational experiments. 


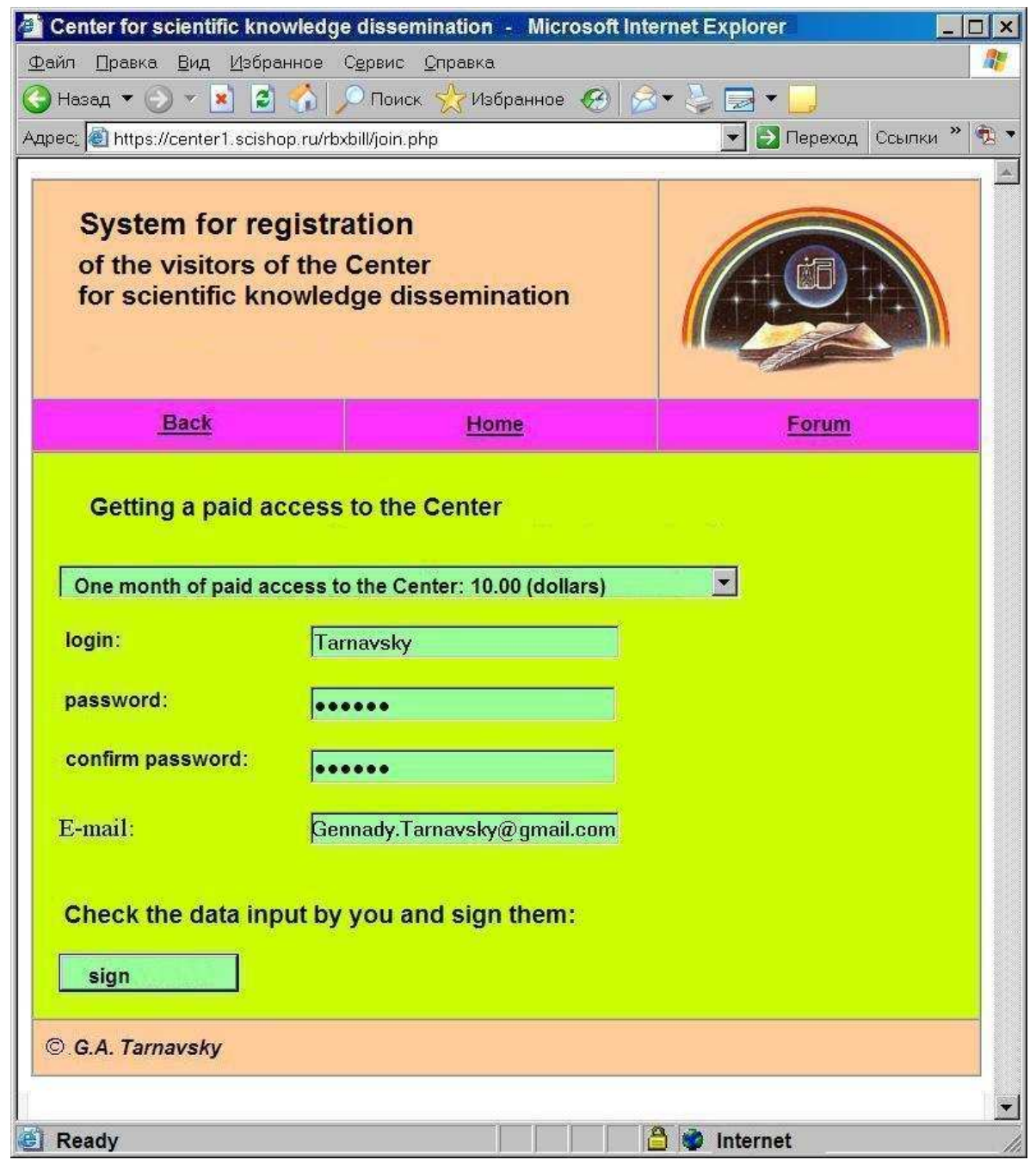

Figure 3: Base page of the site section "The system for registration of the visitors of the Computer Simulation Center".

ICC "Shock" (Fig. 5). A wide spectrum of the investigations of the shockwave flow patterns arising at the hypersonic scramjet engine inlet was done in the works [3, 4] with regard for the variation of the gaseous medium properties on shocks. The configuration of shocks, the type of their interaction (the Mach and/or regular type) and the flow parameters between the shock fronts under the variation of the flight altitude in the Earth atmosphere from 0 to $100 \mathrm{~km}$, the flight velocities from 1.5 to $7 \mathrm{~km} / \mathrm{s}$, the diffuser angles from 0 to 50 degrees were determined.

ICC "Flow" (Fig. 6). The investigation of steady and unsteady flows of both ideal gas (the model of Euler equations) and viscous, heat-conducting gas (the model of full Navier-Stokes equations) around the bodies of different configurations was carried out in the works [5, 6] on the basis of specially developed methods and numerical algorithms within a wide range of determining parameters. The flow structures near the forebody, above the body lateral surface and the body base as well as the flows in the near and far wake of the body were investigated. The characteristic and peculiarities of these structures were determined depending on flight regimes, including the case of 


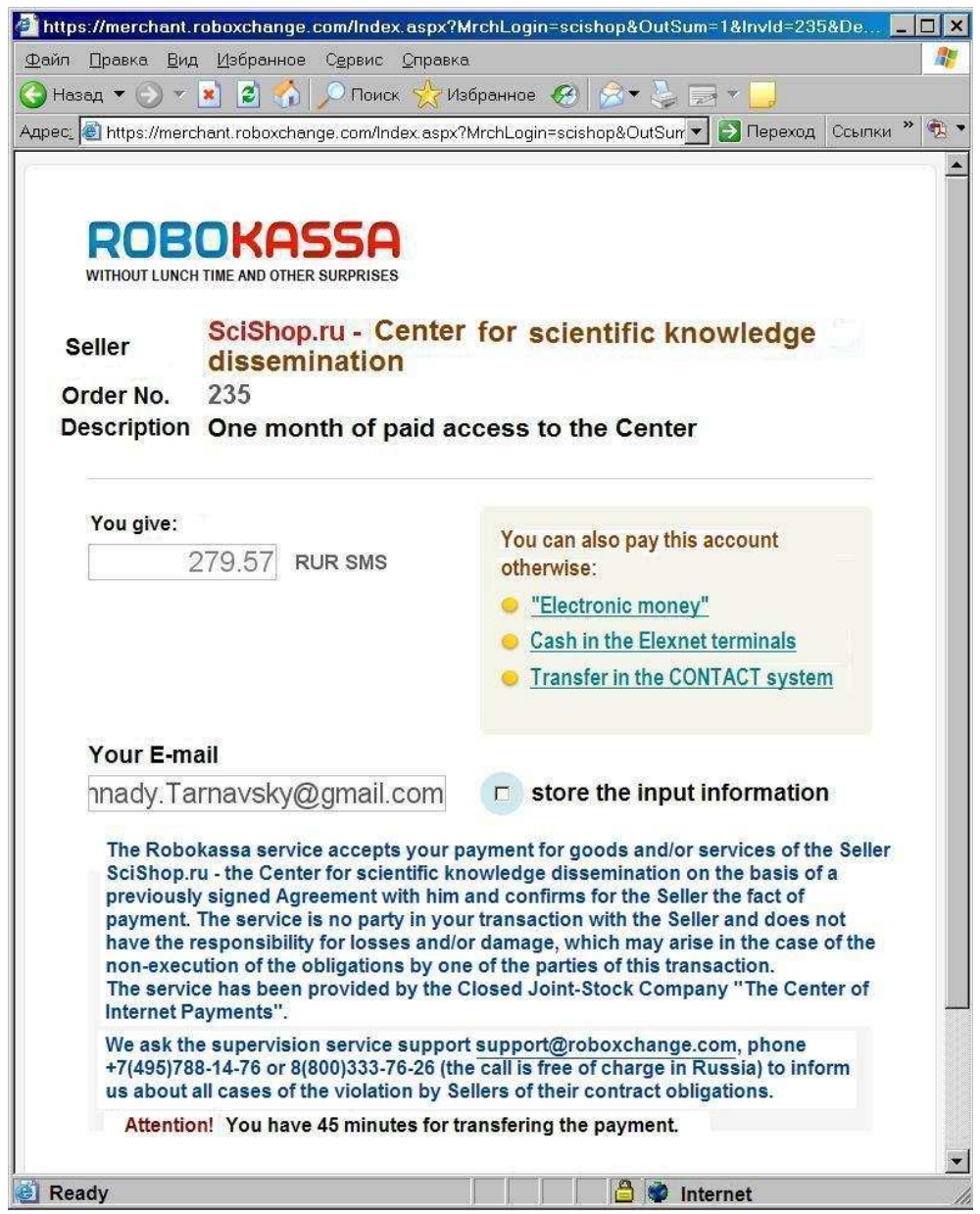

Figure 4: One of the pages of the site section "Transfer of user charge via the SMS messages of cellular communications".

a localized energy supply to the free stream with the formation of a complex flow pattern of the arising internal shock waves, which are determined by the frequency of the sequence of external source pulses, which may lead to resonance phenomena when an insignificant power of pulses gives rise to a substantial destabilization of the flow, high force and thermal loads on the body surface.

The complex enables the modelling of the aerodynamics of the supersonic and hypersonic flight in the Earth's atmosphere at the altitudes from 0 to $100 \mathrm{~km}$ within the speed range from 0.8 to 10 $\mathrm{km} / \mathrm{s}$ as well as, to a certain accuracy (in accordance with available data), a high-velocity flight in the Mars' atmosphere.

In these works, the most important problems of the adequacy of the mathematical model and the algorithms and codes realizing it to the occurring physical process were studied at a qualitatively new level. Three segments of this question, which are related to the nonuniqueness, were considered: the analytic solutions of the Euler equations, numerical solutions of the full Navier-Stokes equations, and the symmetry loss in symmetric problems. The processes of the evolution of the pattern of the flow around the body with the possibility of a passage from one solution branch to another were studied, and the attraction basins of solutions were determined. The trajectories of the computation motion from the starting solution to the final (steady, quasi-steady, unsteady aperiodic) solution in 


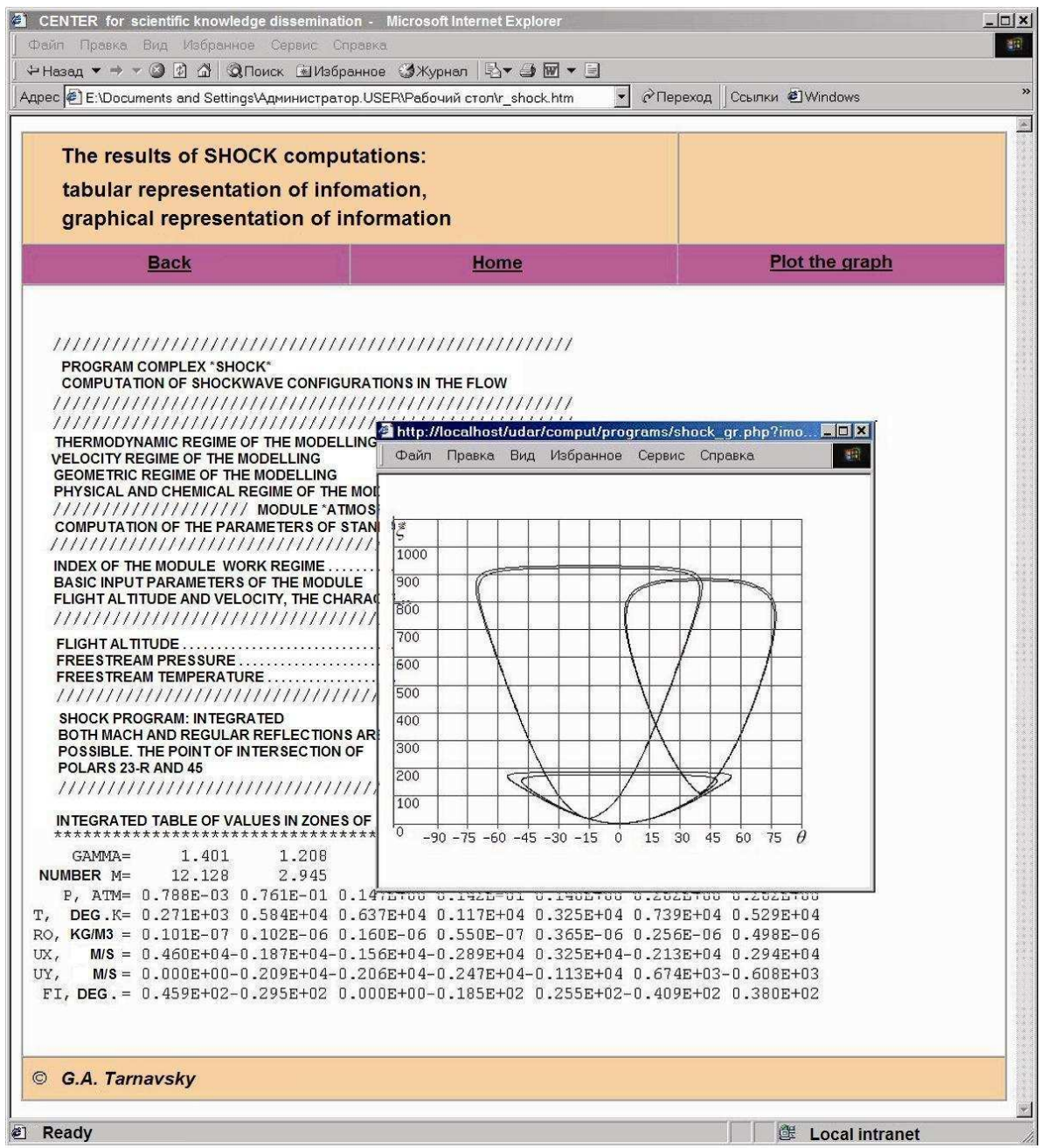

Figure 5: Information-computation complex "Shock". Tabular-digital and graphical representation of the results.

the space of solutions were studied.

ICC "Astra" (Fig. 7). The physical, mathematical, and computational problems of modelling the unsteady three-dimensional problems of the extraterrestrial gas dynamics were considered and analyzed in the work 7 . The system of the Euler gas dynamic equations, which was completed by the force and energy components to model the deviation of the equation of state from the ideal one, the heat-transfer processes (heat conduction, convection, and radiation), gravitation (the gravity field of the point mass and the self-gravitation of a distributed gaseous cloud), was used as the governing system of the differential equations of the mathematical model. The modelling was carried out on the basis of the principle of the decomposition of the complete problem into several sub-problems corresponding to different physical processes. The structuring of the computational complex into several autonomous segments, in its turn, corresponded to this decomposition. This ensures the possibility for extension and supplement of the package of computer programs. The series of the computations of problems on the motion of shock waves and expansion waves in intergalactic gaseous media, on the gravitational collapse of motionless and rotating gaseous clouds, the recession of a 


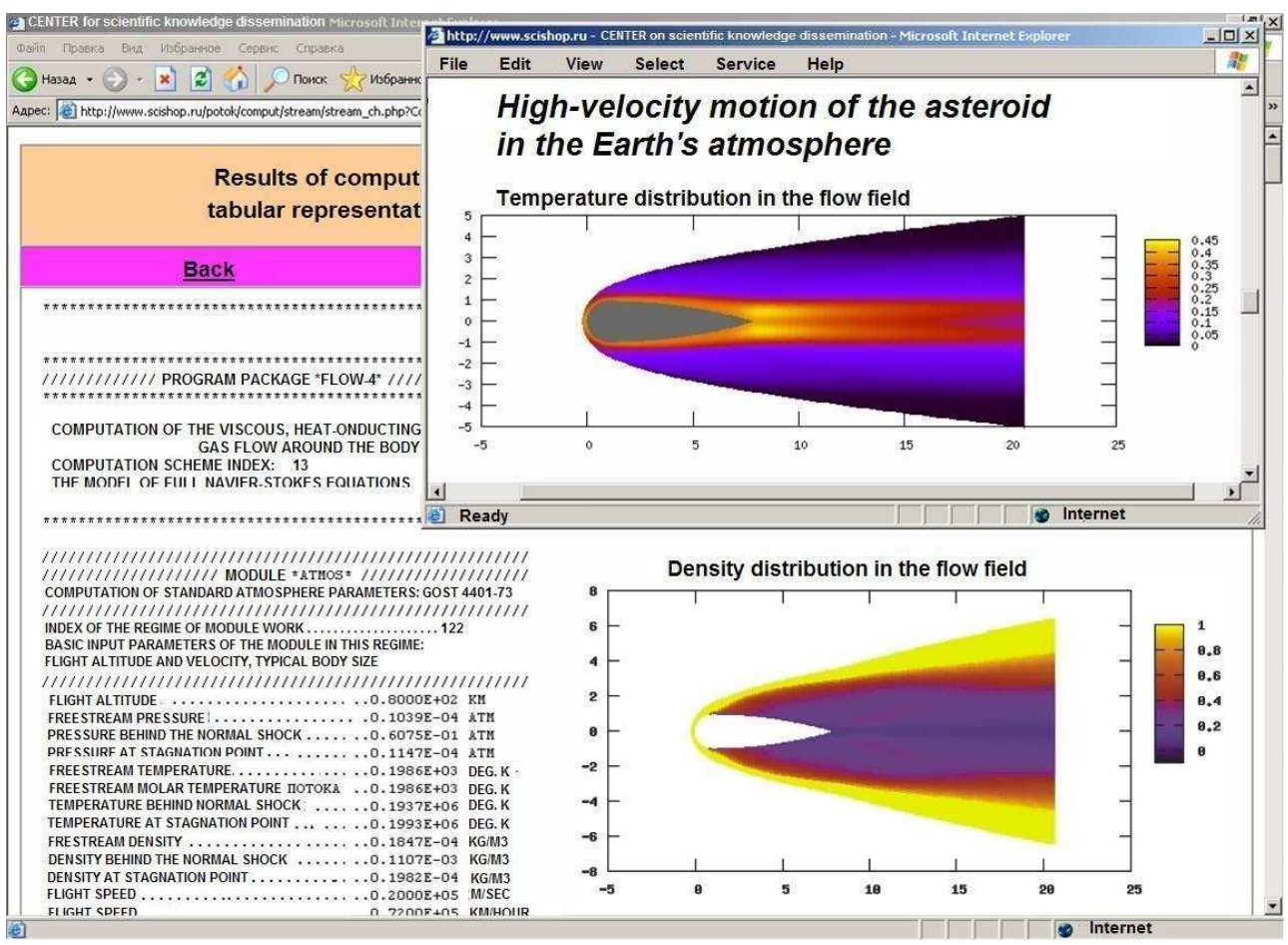

Figure 6: Information-computation complex "Flow". Tabular-digital and graphical representation of the results.

gaseous cluster, which model the formation and explosion of proto-stars, were done. A thorough verification of the theoretical method, the computational algorithm, and complex of computer codes was done for a comprehensive analysis of their properties (the accuracy of computation and the speed of the computational process).

ICC "Nano" (Fig. 8). Theoretical methods were developed in the work 8 for mathematical modelling of a number of physical-chemical and mechanical processes of the technological cycle of the production of new semiconductor materials, in particular, the motion of the oxidation wave in silicon, including the case of the availability of technological masks ensuring special configurations of the interfaces "material/oxide" with the formation of multiply connected regions. These methods were the basis for developing the efficient numerical algorithms and the complex of computer programs.

Special methods, high-accuracy algorithms, and computer codes were developed for computing the physical processes of the segregation of dopant donor and acceptor admixtures (boron, antimony, and arsenic) at the oxidation wave front in a free-crystalline and prestressed silicon [9].

The computer simulation of the formation of specific nanostructures - narrow localized zones of an elevated electric conductivity of the n- and p-types was conducted.

The complex enables the design of nano- and microelectromechanical systems (diodes, capacitors, transistors, etc.) entering the large, very large, and ultra large integral circuits.

A many-sided experience obtained in the course of the works was implemented in the algorithms of program complexes, which are granted to the visitors of the Center [10, 11] for solving their own tasks in the corresponding knowledge areas. 


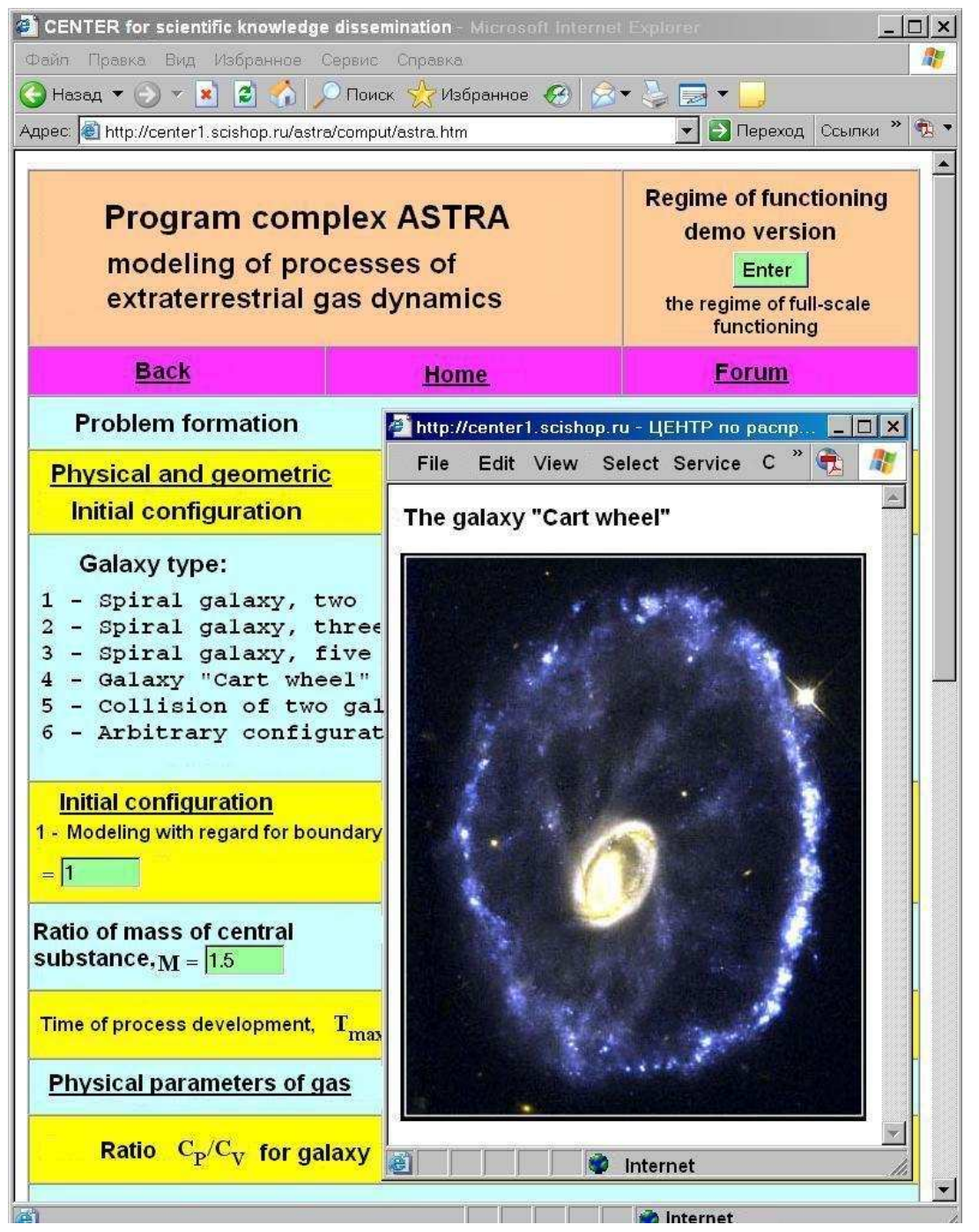

Figure 7: Information-computation complex "Astra". Page for the preparation of tasks and graphical representation of the results.

\section{Conclusion}

The infrastructure and content of the Computer simulation center SciShop.ru, the pioneer of a new form of the scientific knowledge dissemination, were briefly considered in the present paper. This center is intended for a direct use of program complexes for the mathematical simulation of processes in various scientific areas, and it provides the possibility of a direct execution of computations in Internet in the remote access regime. Such a form has wide prospects of the application in scientific research and applied developments as well as for a remote teaching of specialists, postgraduates, and students. 


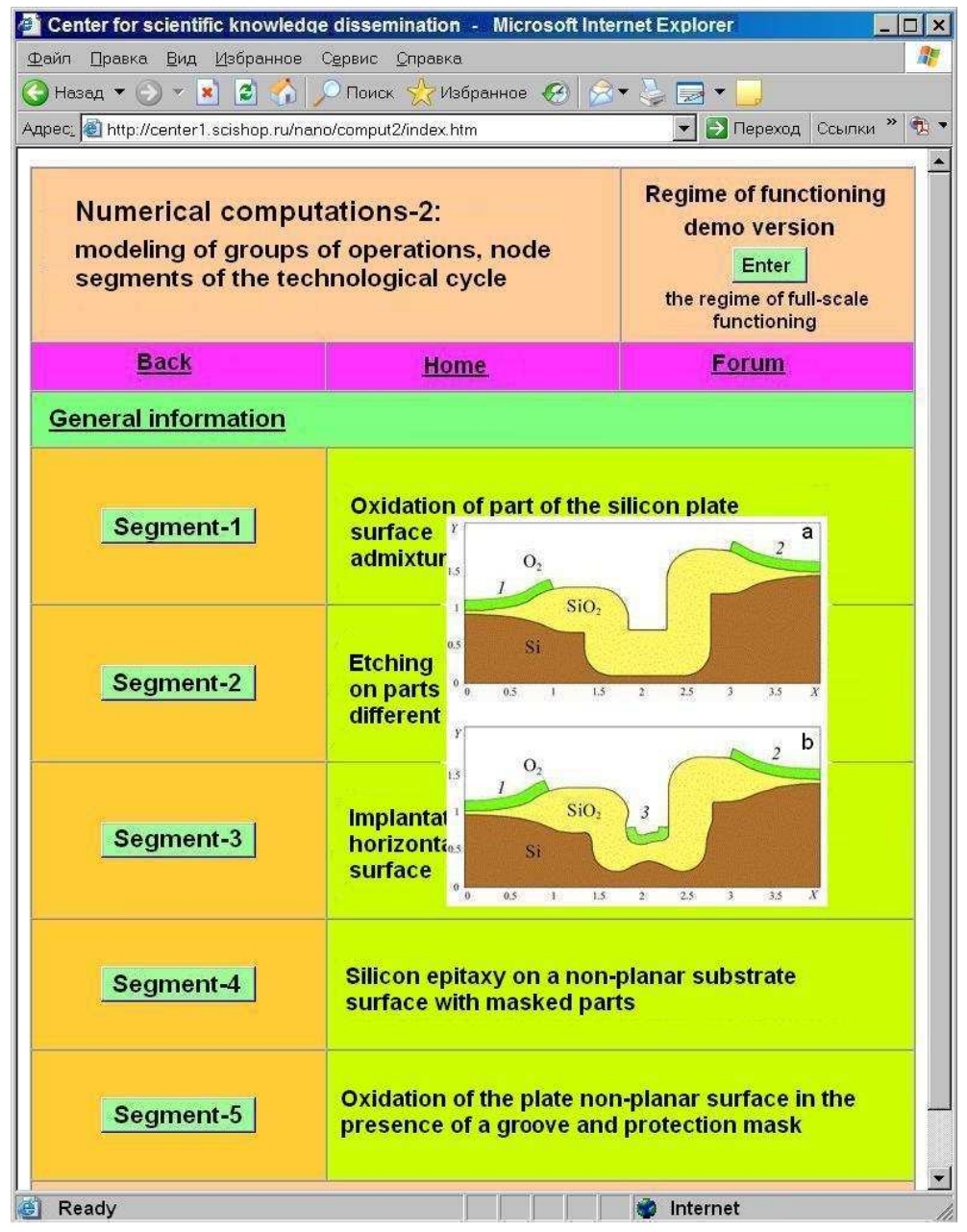

Figure 8: Information-computation complex "Nano". The page of a task for simulation of the groups of technological operations and graphical representation of the results.

\section{References}

[1] G. A. Tarnavsky, A. V. Aliev, V. S. Anishchik, A. G. Tarnavsky, S. B. Zhibinov, and S. S. Chesnokov. Information technologies and problems of the development of the Computer Simulation Center in Internet (in Russian). Information Technologies, No. 8: 68-73, 2009.

[2] G. A. Tarnavsky. Cloud computing: content, infrastructure, and technologies of the arrangement of information flows of the Computer Simulation Center SciShop.ru (in Russian). Investigated in Russia, 13(001):1-29, 2010. http://zhurnal.ape.relarn.ru/articles/2010/001.pdf

[3] G. A. Tarnavsky, A. G. Tarnavsky, and K. V. Gilev. Information-computation Internet Center "Aeromechanics". The first line: program complex "Shock" (in Russian). Numerical Methods and Programming, 6(1):27-48, 2005.

[4] G. A. Tarnavsky. Shock-wave modes of flow at the inlet to the diffuser of a hypersonic scramjet engine: The effect of flight altitude and velocity. High Temperature, 43(1):58-72, 2005. 
[5] G. A. Tarnavsky, A. V. Aliev, and A. G. Tarnavsky. Computer simulation in aeromechanics: program complex "Flow-5" (in Russian). Aerospace Technology, No. 4:27-38, 2007.

[6] G. A. Tarnavsky and A. V. Aliev. Peculiarities of the high-speed flight aerodynamics: computer modelling of hypersonic flow around the object forebody (in Russian). Numerical Methods and Programming, 9(2):371-394, 2008.

[7] A. V. Aliev and G. A. Tarnavsky. Hierarchical SPH-method for mathematical modelling in gravitational gas dynamics (in Russian). In Siberian Electronic Mathematical Proc., 4:376-434, 2007.

[8] G. A. Tarnavsky and V. S. Anishchik. Toolbox NanoMod for computer support of the design of nanostructured semiconductor materials (in Russian). Numerical Methods and Programming, 10(2):34-50, 2009.

[9] G. A. Tarnavsky and E. V. Vorozhtsov. Dopant implantation into the silicon substrate with non-planar surface. Energy and Power Engineering, 2(2):73-77, 2010.

[10] G. A. Tarnavsky. Remote computer simulation of shockwave structures in hypersonic gas flows: technology of cloud computations "The workplace as a service" (in Russian). Numerical Methods and Programming, 11(2):1-25, 2010.

[11] S. B. Zhibinov and G. A. Tarnavsky. Computer Simulation Center in Internet: problems of copyright and intellectual property of the content (in Russian). Investigated in Russia, 12(073):953967, 2009. http://zhurnal.ape.relarn.ru/articles/2009/073.pdf 


\title{
How are white squirrels able to survive in a forest?
}

\author{
J.C. Hodge ${ }^{1 *}$ \\ ${ }^{1}$ Blue Ridge Community College, 100 College Dr., Flat Rock, NC, 28731-1690
}

\begin{abstract}
The white squirrels' (Sciurus carolinensis) causes of success in a preditory environment and causes of the apparent population equilibrium with gray squirrels are mysteries. White squirrels of Brevard, NC are thought to be a color variant of the Eastern gray squirrels. White and gray squirrels were observed from 2001 to May 2010. The squirrel population in the observation area has changed from predominantly gray to predominantly white. The observations suggest white squirrels have many physical and culture characteristics that differ from gray squirrels. These characteristics favor white squirrels over gray in a substantial feral and stray cat (Felis catus) predation and human environment. That Brevard white squirrels may be at an evolutionary branching point is suggested.
\end{abstract}

White squirrels; prey preference; evolutionary dynamics

\section{INTRODUCTION}

Because white pelage is much more noticeable to predators in a forested environment, the cause of the success of the white variant is a mystery. Human protection may be part of the answer.

White squirrel colonies exist in various places in the eastern US (Glesener 2010). The white squirrels of Brevard, North Carolina are distinct from other white squirrel variants. The white squirrels of Brevard have dark eyes, a dark patch on their head, a wide shoulder patch called a saddle, and a dorsal stripe. The dorsal strip occasionally extends the length of the tail. The white squirrels are postulated to be a color morph of the Eastern gray squirrel. However, no head patch has been observed in normal gray variants. Also, a seasonal variation of head patches ranging from gray to pure black has been observed. The inheritance pattern of the two morphs is unknown. The white and gray morphs appear to segregate as separate Mendelian units (Glesener 2001a).

The only other colonies of Brevard type white squirrels are in the southeastern US, particularly in Florida. These colonies appear to have a direct

\footnotetext{
*E-mail:jc_hodgeblueridge.edu
} 


\section{INTRODUCTION}

connection to the Brevard white squirrel (Glesener 2010). Other white squirrel colonies are true albino or white with dark eyes but without the head patch, saddle, or dorsal stripe.

Local folklore holds that white squirrels in Brevard originated from islands off Hawaii or from China and were brought to Florida by a circus. The Brevard white squirrel descended from a single pair trapped in northern Florida and released in Brevard in 1951. The Hawaii or China origin story may have been invented to enhance the appeal of the folklore. Since release, the Brevard white squirrel has increased in abundance and disbursed over a wide area from Cashiers to Hendersonville, a span of approximately 50 miles. Admiring humans may have assisted this distribution.

Periodic sample censuses are conducted (Glesener 2001a,b). This census investigates the relative abundance of white to total squirrel population (hereinafter "Ratio") in an approximate three-mile radius centered on downtown Brevard. The census methods would miss or underestimate some effects such as environmental factors, how far squirrels will travel for a sudden large availability of food, daily activity variance, culture characteristics, and characteristics with short survival times.

Glesener (2001a) reported the Ratio had remained relatively constant at between $21 \%$ and $25 \%$ for several years. Since then, the census has found the Ratio may be increasing. The April 2010 census found an estimate Ratio of $36.7 \%$, the highest on record, and a 12-year average Ratio of $27.4 \%$. However, Glesener (2010) noted this change in the Ratio might be caused by changes in the count protocol. Also, the error bars indicate the recent Ratio increase may not be significant. Glesener (2010) is confident that the white squirrel population is doing well and that the Ratio appears stable. This is another part of the white squirrel mystery.

Glesener (2010) noted variation among the census sectors of both the total number of squirrels and the Ratio. This suggests that environment affects the Ratio in addition to the number of squirrels in a sector. Notably, the highest Ratio (88.9\%) and low overall squirrel abundance were observed on the Brevard College campus. The Brevard College campus has several buildings with moderate, open, and grass covered grounds and sparsely placed trees.

Brevard has a large stray cat population that is considered a nuisance. The cats hunt and kill squirrels.

Neither a white dominant model nor white recessive model describes the apparent Ratio equilibrium (Glesener 2001a). This inconsistency between calculations of gene frequency and census observations suggests the existence of some undetected pleiotropic effect. White squirrels and gray squirrels freely interbreed and have mixed litters. Current wisdom holds that the offspring of mixed mating are either white or gray, but not blends. Glesener (2001a) postulated a gene frequency model to explain the apparent Ratio equilibrium.

This paper suggests that there is a constellation of characteristics including genetic traits, culture, and behavioral traits that allow the Brevard white squirrel to have a higher fitness than the gray squirrel in a highly predatory environment. The white squirrel is white, is cautious, is more agile, has a smaller 


\section{OBSERVATIONS}

body structure, and has a shorter and less bushy tail. The gray squirrel is gray, is more aggressive, is less agile, has a slightly larger body structure, and has a longer and bushier tail. White squirrels and gray squirrels have different cultures. Individual survival requires all the characteristics of the variant be present. That is, the mixing of culture and pelage characteristics within an individual is selected against. Indeed, the highly predatory environment such as in the high stray cat population of Brevard is necessary for the white squirrel to be more fit. The cats introduce a prey preference than causes a stable Ratio.

In section 2, the observations are described The Discussion and conclusion is in section 3 .

\section{Observations}

A bird feeder was installed in 2001 on the east side of 16 Hosta Lane, Brevard, North Carolina 28712 to feed and watch birds. This site was approximately one mile outside Brevard city limits and was outside the squirrel census zone. This feeder has become a squirrel feeder. The food was sunflower seeds. The feeder was suspended approximately 1.5 meters above the ground and one meter from a tree, which was at the edge of a sharply declining slope of approximately 40 degrees. Food dropped to an area of approximately one meter diameter on the ground (hereinafter "Food Area"). The feeder was located approximately three meters from a sliding glass door. The "Feeding Volume" included the feeder, the Food Area, and three meters distant from the feeder. The "Feeding Area" was on the ground within three meters of the Food Area.

Other than stocking the feeder and being close, I did not interfere or interact with the critters. Several patterns and characteristics of white squirrels and gray squirrels have been qualitatively noted.

Distinguishing individuals by their gray pattern determined the number of white squirrels. The number of gray squirrels was determined by distinguishing individuals by their body and tail size. The maximum number that was seen simultaneously within a week determined the number of chipmunks (Tamias striatus) visiting.

Initially, one white squirrel visited once or twice per week. Up to ten gray squirrels visited the Feeding Volume daily. One to two chipmunks visited the Feeding Volume daily.

The number of chipmunks visiting has cycled between none and six. The number of stray and feral cats (herein simply "cats") cycled between none and two. Domestic cats were discounted because they were too fat and slow to be a serious threat to the squirrels. However, they did harass squirrels. The cat cycle lagged the chipmunk cycle by approximately two weeks after I saw four chipmunks. Domesticated dogs were running loose in the area - a situation that may not be present in Brevard city. Also, hen hawks (Accipiter cooperii) and, occasionally, Red-tailed Hawks (Buteo jamaicens) frequently appeared in the sky and trees.

The daily visit count of eight or nine white squirrels has existed since 2007 . 


\section{OBSERVATIONS}

Eight active drays were within an 80-meter radius in the summer of 2009 (Rowe 2009). This was the same number of human houses in this area. This implied approximately 2.8 squirrels per forested acre. Because food was supplied daily, perhaps this was a squirrel culture limit rather than a mast limit. A neighbor also feeds squirrels. When he left for more than a day, over 18 white squirrels visited daily. Therefore, squirrels routinely traveled 80 meters to a food supply and 160 meters when food was scarce. Apparently, a constant and copious mast supply bred more white squirrels not bigger white squirrels. That is, smaller size was an advantage because the group culture structure can be more effective with more individuals for a given food supply.

By May '10, an average of one to three male gray squirrels visited approximately twice per week. Five female and six male white squirrels visited daily. Up to six white squirrels within the viewing area were seen simultaneously daily. Twice in 2008, in Jan. '09, and in Mar. '10 nine white squirrels were seen simultaneously.

The larger gray squirrels had a larger body size, a longer stride, a longer tail, and a wider, bushier tail than the largest white squirrel. The medium sized gray squirrels also had longer and bushier tails than most white squirrels. The larger white squirrels chased and caught the smaller gray squirrels. The medium and larger sized gray squirrels chased all white squirrels. However, a gray squirrel catching a white squirrel has not been observed. Usually, the white squirrel gained at least a half-meter after a three-meter chase. Among squirrels, the squirrel with the larger tail size chased the squirrel with the smaller tail.

The feeder usually emptied each day. The limit of view in the forest during summer was approximately 50 meters. After the feeder was refilled, from five minutes to two hours (to normal feeding time) elapsed before the first squirrel arrived. The time lag was dependent on when the feeder was refilled and which morph arrived first. The shortest time to first squirrel was just after sunrise, around midday and late afternoon. If the feeder was refilled between these times such as at $10 \mathrm{AM}$ and if the first squirrel to visit was a gray squirrel, the next squirrel arrived an average of 40 minutes later. Up to two hours may elapse before the next squirrel arrived occasionally occured. Other squirrels in the trees between 20 meters and 50 meters did not come to the Feeding Volume.

If the first squirrel was a white squirrel, the next one to four squirrels arrived within 20 minutes. Other squirrels in the trees between 20 meters and 50 meters did come to the Feeding Volume.

The definition of a "dominant" squirrel in a group was refined in 2009 to mean high in the pecking order, only. Physical fights ultimately determined pecking order. However, tail size appeared to substitute for fighting.

The definition of an "aggressive" cultural characteristic was refined to mean a squirrel behavior wherein the squirrel either did not tolerate other squirrels within a half-meter radius when feeding or left the Feeding Area when other squirrels were present which applied to squirrels low in the pecking order.

The definition of a "cautious" cultural characteristic was refined to mean a squirrel behavior wherein the squirrel was tolerant of others in the Feeding Area. A cautious, dominant squirrel in the Feeding Area chased other squirrels 


\section{OBSERVATIONS}

for a short distance if they approached within a half-meter or tolerated the others in the Feeding Area. Other characteristics included more wariness than aggressive squirrels of new things, of new noises, and of being too close to a place where it could not see such as corners. For example, the cautious squirrel slowly approached a new dead stick in the Feeding Area whereas the aggressive squirrel usually did not approach or investigate. When startled, the cautious squirrels climbed higher and returned slower than the aggressive squirrels.

The set of squirrels that were aggressive and the set of squirrels that were cautious were mutually exclusive sets. Each squirrel was in one of these sets. A dominant squirrel could be either cautious or aggressive. Likewise, a squirrel low in the pecking order could be either cautious or aggressive.

As long as there were three or less squirrels on the ground, the cautious, dominant squirrel can maintain the half-meter feeding radius. More than three gray squirrels within the Food Area have not been witnessed. More than three and up to six white squirrels in the Food Area simultaneously occurred frequently. The squirrels lower in the pecking order occasionally tested the more dominant squirrel. If there were three or less squirrels in the half-meter zone, the cautious, dominant squirrel attempted to chase or fight. This chasing was little more than a threat if the chased squirrel left or was a chase of less than four meters. That is, the cautious, dominant squirrel asserted dominance then quit.

Occasionally two cautious squirrels shared the feeder. If there were three cautious squirrels in the Feeding Volume, there was a little chasing but they soon settled into an acceptable eating arrangement. A less dominant, cautious gray squirrel was tolerated in the Food Area by dominant, cautious, white squirrels. Two to four cautious squirrels could be on the ground within half-meter of each other feeding. If there were six or more cautious squirrels in the Feeding Volume, one or more would settle on a tree branch and wait.

The aggressive squirrel attempted to chase others in the Food Area. If the other was a more dominant squirrel, a physical fight ensued. The aggressive squirrel was often injured. Another response of aggressive squirrels was to visit the Feeding Area alone at times when others were usually absent. This was an opportunity for predators.

When four or more squirrels were in the Feeding Area, one then another tested the aggressive squirrel's half-meter feeding radius nearly constantly. Eventually, the aggressive squirrel appeared to loose its temper and started chasing the unlucky one of its harassers for several tens of meters. During this chase, which may last a half-minute or more, one or more of the other squirrels invaded the Food Area. When the aggressive squirrel returned, it chased another one of the invaders. This process continued for a few cycles. The aggressive squirrel then retreated to an overlooking branch and waited for the larger number of squirrels to leave. As the aggressive squirrel gains experience, it left the area without the long chase. That is, it was intolerant of others feeding nearby. Further, if an experienced, aggressive squirrel visits when squabbling white squirrels were on the ground, the aggressive squirrel left the area. Dominant squirrels tend to feed on the feeder. When a dominant squirrel was on the 


\section{OBSERVATIONS}

feeder, aggressive squirrels fed on the ground.

Aggressive behavior in a group carried a huge energy price. However, such energy expenditure may have a return if the mast was scarce.

The landscape around the house before a recent remodeling provided two ambush areas for cats that were easily monitored. One was the Feeding Area and one was in the front of the house. The forest around the house provided many other ambush sites. The one in front was a carport. A cat would often hide under the car by the car wheel. When a gray squirrel or chipmunk crossed in front of the house to travel from the Feed Volume to the forest on the other side of the house, they usually traveled close to the house, which was a shorter distance than crossing away from the house. The white squirrels traveled at least five meters from the carport and usually across the narrow part of the driveway. Since monitoring started in 2004, over 30 dead gray squirrels and no dead white squirrels were found within one meter of the carport with a distinguishing bite in the back of its neck.

Cats were frequently seen killing and carrying away chipmunks at the ambush site near the feeder. One white and two gray aggressive squirrels were seen killed in the Feeding Area. They were alone and on the ground. Cautious squirrels had also been seen alone on the ground. However, cautious squirrels were more wary than aggressive squirrels. They sensed the cat approaching before the cat came within striking distance.

Between 2005 and 2009 squirrel road kill between my house and approximately one mile toward Brevard was counted during visits to Brevard. The road was well traveled with forest on both sides of the road. This area seems to have had more white squirrels than gray squirrels in 2005, 2006, and 2007. However, the road kill count averaged 14 gray squirrels and two white squirrels per year. The annual road kill count averaged two gray squirrels and two white squirrels per year in 2008 and 2009 when the white squirrel ratio was high. Because road kill was quickly disposed and because counts were taken only one to four times per week, these counts were likely underestimates of actual road kill.

The nearest, squirrel-supporting tree from the feeder tree was over two meters distant. The white squirrels occasionally jumped this distance rather than travel a longer route. No gray squirrel has been seen jumping this distance. When a gray squirrel was chasing a white squirrel, the white squirrel frequently climbed the tree one to three meters and jumped to another tree approximately one meter away. The gray squirrel did not follow. If the squirrel chasing was a white squirrel, it would frequently follow at this height. However, if the chased white squirrel ascended to over five meters, the chasing white squirrel would follow only infrequently.

If a cat approached, white and gray squirrels emitted a chattering, went up trees, and left the area for at least 15 minutes.

The hen hawks tended to glide toward the feeder from the east. A squirrel feeding on the feeder could not see the hawk approach from this direction. One to three times per week a hawk perched in the forest overlooking a one-lane road. The cautious squirrels stopped feeding and watched. Infrequently, the hawk perched in a tree overlooking a flight path that was an attack path for 


\section{OBSERVATIONS}

the Feeding Area. Two to four times per year, the hawk was seen diving on the Feeding Area. White squirrels in the Feeder Volume emitted a higher rate of chatter than for a cat. Most, but not all, white squirrels scurried under bushes rather than climb trees. White squirrels on the feeder rapidly descended and went under the bushes. Gray squirrels climbed trees. Hen hawks have been known to attack prey in trees. The squirrels returned in two to 10 minutes. The white squirrels apparently had differing signals and behavior for dangerfrom-above and danger-from-the-ground. The gray squirrels did not.

A unique male squirrel started visiting in the early summer of 2005. It had the body but not tail of gray squirrels and mostly gray hair with white on its head, neck, and side. It was a blend, one of two noted during the observation period. It was an aggressive squirrel. This squirrel stopped visiting by mid summer.

Each year one or two younger, white squirrel(s) with variation visited. Some had difficulties or limited abilities. One such white squirrel appeared normal except for a behavioral trait difference in the summer of 2009. Being young and small, other white squirrels would chase it from the feed. It would position itself in a tree close the food and give the danger-from-below call. The other white squirrels scampered up the trees and soon left. The false caller would then eat. After a few weeks and when the false caller had given a warning, the other white squirrels looked but did not leave. A few weeks later, an incident occurred wherein the false caller was on the ground eating and one other white squirrel was on the feeder. The white squirrel on the feeder was gathering seeds and then went to the top of the feeder to eat. Suddenly, the white squirrel on the feeder left the area unusually rapidly without calling. Within two minutes a cat attacked the false caller. The false caller escaped. Within two weeks, the false caller stopped visiting. This was the only squirrel during the observation period with this characteristic.

An aggressive, female white squirrel with a white tail except for distinguishing dark fur at the end of its tail was in a fight with a larger white squirrel in May '09. This aggressive squirrel limped away. The next day this squirrel had a swollen right front paw. A week later, it was missing its right front paw. Its tail became thin. It ate only when other squirrels were absent. It survived through 2009 and became stronger with a bushier tail. By May '10 it could chase smaller white squirrels and maintained its aggressive personality.

As of May '10, two of the 11 white squirrel daily visitors were aggressive. Usually none or one aggressive white squirrel was seen in a season. Both aggressive white squirrels were in the middle of the pecking order. Two of the three male, gray squirrel weekly visitors were aggressive. Both were at the top of the pecking order. The cautious gray squirrel was near the bottom of the pecking order.

A black and white, stray cat remained near the house in the summer of 2009 . Usually the stray cats left when the number of chipmunks declined. By Nov. '09 there were no chipmunks noted in the Feeder Area or around the house, which was unusual. This cat was seen with an injured right front leg in early Nov. '09. This cat was last seen in mid Nov. '09. Since then, no feral or stray 
cats have been seen.

\section{Discussion and conclusion}

The gray squirrels evolved in a wild, forested environment. The gray camouflage allowed the dominant squirrels with increased mating opportunity to be aggressive. The white pelage is very noticeable in the forest. Therefore, developing a cautious characteristic with a white pelage seems plausible a posteriori. Further, developing the cultural characteristic of tolerance in larger groups in a concentrated mast supply seems plausible. Also, a smaller size would allow survival of more individuals for limited mast and for years of low mast availability. All these changes are difficult to explain in a step-by-step evolutionary model in an environment that has selected the gray squirrel.

A human involvement in the selection process is a simpler explanation. Because the initial record was of a circus, the white squirrels may have been selectively bred for the white trait. Selective breeding for wider white underbelly within an enclosure may have also bred for less aggressiveness, more caution, and willingness to feed in larger groups. The foreign origin folklore may have been a circus promotion embellishment. Further, the white squirrel's slighter body, more agility, with more developed speech such as the noted warning sounds and, perhaps, a more developed culture structure such as indicated by the number of squirrels around the feeder simultaneously may be their key to survival in an area of substantial predation.

The present observations suggest a different dynamic of the Ratio than gene frequency of only a pelage trait. The varying Ratio among count sectors (Glesener 2010) and the present observation of a changed Ratio upon the introduction of the feeder and the removal of a cat kill zone suggest the Ratio is environment dependent. Post et al. (2000) studied the dynamics of two noninteracting pray populations in an environment where the predator kills in response to pray frequency. They found that although chaos is possible in food webs, pray preference reduces the potential for chaos. The pray preference may be exercised by the pray. The cautious behavior reduces the cats' opportunity and aggressive behavior may trigger the cats' kill response. This predator-pray dynamic may result in a stable Ratio. Berezovskaya et al. (2009, and references therein) argued that evolution should favor heterogeneous settings including Allee effects. Doebeli \& Ispolatov (2010) demonstrated that evolutionary diversity could occur after the population reaches a continuous stable strategy such as demonstrated by a stable Ratio in a stable environment.

\section{Acknowledgments}

I appreciate the financial support of Maynard Clark, Apollo Beach, Florida, while I was working on this project. 


\section{REFERENCES}

\section{References}

Berezovskaya F., Wirkus S., and Castillo-Chavez C. 2009. Predatorprey interactions in communities with prey dispersal and Allee effects. http://arxiv.org/abs/0907.5382.

Doebeli M. and Ispolatov I. 2010. Continuously stable strategies as evolutionary branching points. http://arxiv.org/abs/1005.3862

Glesener R.R. 2001a. Change in gene frequency in an urban eastern gray squirrel population. J. Elisha Mitchel Sci. Soc. 117:199.

Glesener R.R. 2001b. Equilibrium of Brevard NC's white and gray variants of the eastern gray squirrel. J. Elisha Mitchel Sci. Soc. 117:199.

Glesener R.R. 2010. Overview of findings. http://www.brevardnc.org/whitesquirrel/

Post D.M., Conners M.E., and Goldberg D.S. 2000. Prey Preference by top predator and the stability of linked food chains. Ecology 81:8.

Rowe A, 2009. Personal communication. 


\title{
Customers' Motivation as the Basis of Effective Forming of Communication Mix
}

\author{
N.Skrygun, O.Krainyuchenko, O.Bezpalko \\ National University of Food Technologies, Vladimirskaya str. 68, \\ 01033, Kyiv-33, Ukraine
}

\begin{abstract}
Essence of complex of promotion and his elements is considered, grounded necessity account of type and level motivation of consumer at forming of communication mix, the types of consumers are selected taking into account the level of their motivation, which allows developing effective strategy advancement of the product.
\end{abstract}

Keywords: promotion,communication mix, consumer, motivation, advertising, need.

\section{Introduction}

The inalienable constituent of marketing complex are marketing communication, role and the value of which is acquires the special actuality at forming of favour and loyalty of consumers to the firm's commodity. Tha's why the marketing is considered as a system of communication that by means of the use of psychological mechanisms of influence on consumers' behavior and activate objective actual or potential necessities. The meaningfulness of forming of effective marketing communication is explained and that they try to co-operate customer with a salesperson, provides the individual approach to a potential consumer with taking into account of his psychological features, carry out strengthening and even (at a necessity) change the level of motivation. Therefore, in the article approaches

purchased further development in relation to creation of high-efficiency facilities of advancement on the basis of psychological analysis of consumers' types and their personality characteristics.

\section{Formulation of the problem}

The increase of advertisement communication efficiency need the deep analysis of types of consumers, personality descriptions with the help of which it is possible to create exact and high-efficiency marketing communication psychologically, and also to design goods and services, that are the most needed by the customers. However, there are some 
disadvantages during the reporting of both economic and psychological character that considerably reduces efficiency of communication mix. As the achievement of communication aim is in the sphere of consumer' psychology, the marketing communication must form psychological options that would assist and try to achieve marketing communication targets.

The problems of development of the theoretical bases and different aspects of effective practical application of complex of marketing communication in economic activity of the enterprises were investigated by such home and foreign scientists: S.Garkavenko, T.Lukiynets, T.Prymak, Y.Romate, P.Smith and other. The researches of factors, that influence on consumers' behavior found a reflection in scientific works of J.Angela, O.Zozuliova, O.Lebedeva-Liybimova, A.Starostina and other. Thanks to the works of the scientists, it should be noted that separate questions in relation of forming of communication mix with taking into account of different type and level of consumer' motivation need clarification and further study. The Uncompleteness of scientific development in relation to the indicated problems define the aim of this research, that consists of the selection of different types of consumers taking into account their personality features and levels of motivation for the sake of development of suggestions in relation to forming the possible strategy of advancement of commodity.

\section{Results}

The system of marketing communication - one of the main instrument of marketing complex by means of which the enterprises influence on a marketing environment. Thus, the question of classification of forms of marketing communication in modern literature remains debatable. That's why, Y.Romate offers the system of marketing communication that can conditionally divided into two groups: basic (advertisement, direct-marketing, sales promotion, public rilations) and synthetic (branding, participating in exhibitions and fairs, sponsion, marketing communication are integrated in the places of sale). The author proves that informal verbal marketing communication (generating a communicator rumours) occupy separate position and can be attributed neither fixed nor to synthetic assets of marketing communication [4, p. 140]. S.Garkavenko complements the basic elements of communication mix with the personal sale, distinguishing it's in independent elements from direct-marketing $[2$, p. 410]. 
Also there is a division of measure on commodities promotion on ATL (advertisement in Mass Media and external advertisement) and BTL- actions (measures on sales promotion, public relations, advertisement in the places of sale, direct-marketing, sponsion and others like that). Some specialists (F.Jefkins, F.Kotler and other) according to independent facilities of marketing communication take packing and so-called collateral (side, second-rate) facilities (postals, advertisement souvenirs and others like that). Now there are more than twenty different elements of marketing communication. We are noticed that a clear limit between the different elements of communication mix sometimes it is extremely difficult to set, as all the marketing communication facilities are effective only at their complex use (effect of synergy). Thus, outlets contain cutting or torning off coupons, that, as a rule, give a right on a discount from a price at a purchase promoted product, that often is one of forms of sales promotion or to contain the elements of public relations. Direct-marketing that is realized by the method of direct mail can use outlets, standards promoted product (so-called sampling, which is attributed to sales promotion) and others like that.

Marketing communication substantially differ both in a technological and in organizational plan. The application of psychological processes of a consumer, his/her perception, attention, memory, emotions, process of taking decision about a purchase, motivations, necessities, reasons, stereotypes, values and others like that helps marketing specialists to design the reaction of consumer on marketing stimuli, that they are carried in itself marketing communication. A main task consists of the determination of correlation of the pre-arranged influences with objective necessities and possibilities of a consumer, with its numeral psychological features and psychical processes.

At forming the complex of marketing communication it is necessary to take into account a type and level of motivations of a consumer, in fact the strategy of communication mix depends on it. On the basis of the conducted analysis of possible consumers' types and levels of their motivation is developed a possible strategy of commodity advancement. The first type - is active customer with the brightly expressed and realized requirement in a commodity. In this case a consumer can make the active search of concrete object for satisfaction his necessity (commodity or services), or to understand that these commodity or services are really necessary for him, goods and services from a certain commodity group on principle "winter clothing", "transport", "flour pastry wares", "dairies" and others like that. In 
the first case an advertisement can be examined by a consumer as necessary information about the methods of commodity purchase, and it will be actively and purposefully perceive it (to look through the press, catalogues, to search necessary firms and others like that). Such searching of information is named active. In the second case a consumer is on the stage of decision-making and that's why marketing communication helps or induce to do a choice.

If the given presence of requirement is in a commodity group, the task of marketing specialist is that he must "give some characteristics" to the commodity, to influence at customer' choice (for example, by comparison of separate commodities in this category). In the case when a consumer chose a concrete commodity as method of satisfaction of certain base necessity, marketing communication must be sent to the grant it the objective information in an optimal volume and maximally accessible kind in relation to a presence, property, unique feature, price, place and conditions of sale and other characteristics of commodity. The researches are showed that in this case even sufferet mistakes at promoting product do not influence enough on the high level of consumer estimation of given information as a customer need its not less than the commodity. It explains why an amateurish advertisement works. Thus, excessive methods of persuasion, influence, bringing in, suggestions and others like that can be perceived by an active customer as superfluous that cause an irritation and interfere with an acceptance already made decision. An active consumer has an increase psychological sensitiveness to the advertisement, easier perceives mistakes that complicate communication. Having strong motivation, he finds in an advertisement exactly what he need, pays attention on necessary commodities, distracted from second-rate, obtrusive.

The second type - a customer does not have the realized necessity, but set possibility of unrealized (potential) necessity, that a customer has internal and external terms for his/her origin through the set time. In such case marketing communication will be effective, if they concentrate on the vivid sphere of consumer and his memory. As a rule, the communication mix directs for the reiteration of information; detailed explanation of how the commodity removes the problems of the customer; comparing of commodity advantages with the analogical commodities be means that does not conflict with a current legislation the aim of which is creation in the customer right character of commodity, that can effectively satisfy a potential necessity. For example, a person that does not suffer a disease the medications from 
that are widely advertised, can remember about them, if an advertisement helphim/her to memorize the name. In its turn, it will act part at the choice of medications, if illness will appear. At the level of complex marketing communication a psychological effect largely will be determined by "correct strategy of measures of public relations, as exactly they are sent not to the direct sale, but on creation of the protracted positive character of commodity of firm" born in a mind [3, p. 241]. Creation of proof positive character is provided through memorizing, recognition of trade marks and forming of positive attitude toward them of potential consumers.

The third and most difficult for marketing specialists type of consumer envisages the consumer' absence not only the brightly expressed concrete or potential necessity but also internal terms for its origin. For example, for a person that does not hear, there can not be a necessity in buying audiotechnique, but diabetic person will not buy sweets with content of sugar. Therefore, an advertisement that will call to realization of purchase the commodity inaccessible or unnecessary for these people on objective reasons will cause an irritation and feeling of inferiority as all the time will remind them of present disfigurations. Certain success on such conditions can be scored forming such marketing communication that will suggest by means of promoted product to satisfy certain additional necessity. Thus, a customer can buy objectively an unnecessary for him commodity in a gift, for further resale and others like that.

\section{Conclusions}

Those marketing measures become effective only when they take into account human necessities and objective terms, and realized through the use of modern instruments of marketing communication. It is set that marketing communication can satisfy the obvious (realized) and hidden (unrealized) necessities of consumers for condition if examine them as certain part of commodity and that is oriented to the certain group of customers. An analysis showed that marketing communication gain its targets when in the core of their planning understands the necessity of promotion not only the commodity, but also the satisfaction of potential consumer needs. Therefore, taking into account the consumer' requirements, analysis of his/her necessities and level of motivation as a customer is necessary pre-condition at forming of effective complex of marketing communication. 


\section{References}

[1] About advertisement. The Law of Ukraine// Government courier. - 1996. - №137-138.

[2] S.Garkavenko. Marketing. - K.: Libra, 2002.

[3] A.Lebedev-Liybimov. Psychology of Advertisement. 2-nd edition. - St.P.: Piter, 2007.

[4] E.Romat. Advertisement. 5-th edition. - St.P.: Piter, 2002. 


\title{
Когнитивный аспект исследования концептуальной метафоры с эмоциональной составляющей
}

\author{
Alena Soloshenko \\ Кандидат филологических наук, Россия \\ Докторант Université de Strasbourg, France \\ Email: alena.soloshenko@etu.unistra.fr
}

\begin{abstract}
The present paper explores the cognitive and emotional organization of a conceptual metaphor and its components, in particular, the concept of Fear. Nowadays metaphor is presented as one of the significant mental operations, the tool of perception, and understanding of a reality we live in. Conceptual metaphor research is considered as one of the favorable and effective in various areas of cognitive science. The article deals with the implication of a Qualitative Data Analysis (QDA) and research software into a conceptual metaphor investigation, focusing on the legal thematic texts with emotional content. The data collected via a qualitative research helps to semantically enrich the domains of a conceptual metaphor, to expand the basic understanding of this mental notion, to enlarge and specify a set of modern conceptual metaphors.
\end{abstract}

Keywords: concept, conceptual metaphor, qualitative data analysis, legal discourse, emotional sphere

Язык, будучи средством коммуникации, функционирует в сфере речевой активности. Выступая же средством познания окружающего мира, язык используется в качестве когнитивной способности. Все когнитивные способности человека могут рассматриваться как постоянно развивающиеся способности по ориентации и позиционированию человека в мире. В данном аспекте когнитивная лингвистика играет одну из важнейших ролей. 
Когнитивная наука имеет долгую историю, истоки которой прослеживаются со времен античности. В те времена интеллект, сознание и модели мышления понимались и изучались, в основном, в качестве предметов логики, философии и физиологии (Платон, Аристотель, позже Августин Блаженный, Пьер Абеляр). В рамках современной когнитивной лингвистики парадигма исследования изменилась в плане презентации материала, изменился сам подход. К примеру, более четко обозначилась природа актуального, то есть, вещей, феноменов, событий. Она может по-разному отображаться в сознании человека: в одном случае, она может отображаться посредством визуальной картины, в другом - в качестве наивного понятия, в третьем в качестве символа.

Объектом изучения когнитивизма является человеческий разум, мышление и те ментальные процессы и состояния, которые с ними связаны [1]. Особенно важным является взаимодействие процессов восприятия, обработки, хранения и воспроизведения информации об окружающем мире. В рамках данного подхода неизменно функционируют два взаимосвязанных понятия: действительность и сознание. В понимании того, что есть сознание, исходим из постулатов отечественных школ психологии и психолингвистики (А.Н. Леонтьев, А.Р. Лурия, А.А. Леонтьев, В.В. Красных и др.). Сознание есть специфически человеческая форма отражения действительности и высший тип психики (А.Н. Леонтьев). Действительность - это все сущее, материальное и идеальное, реально существующее и воображаемое (в виде, например, воспоминаний о прошлом, мечтаний о будущем, плодов воображения и фантазий), принадлежащее сознанию и лежащее вне его [2].

Именно эти понятия определяют репрезентацию и функционирование одного из ключевых понятий современной когнитивистики - концептуальной метафоры. Ценностный компонент данного понятия определен тем, что концептуальная метафора является важной ментальной операцией, средством познания окружающего мира, то есть, концептуализации, категоризации, оценки и интерпретации воспринимаемого. Наша обычная концептуальная система, посредством которой мы думаем и действуем, в основе своей глубоко метафорична. Зачастую подчеркивается, что метафоры могут структурировать нашу концептуальную систему, что они «встроены» в природу человек (Lakoff \& Johnson). R. Boyle, к примеру, еще давно заметил, что «среди загадок человеческой речи, метафора остается одной из самых непостижимых» [3]. 
Начиная с 1980-х, метафора начала восприниматься как олицетворение когнитивных и ментальных ресурсов человека. Метафоры стали очень влиятельными понятиями в когнитивной лингвистике, а также в ряду других наук, таких как концептология, лингвокультурология, психолингвистика. Много внимания данному феномену уделяли такие выдающиеся исследователи, как Lakoff (1980), Johnson (1980), Núñes (2000), Kövecses (1986), Turner (1990), Fauconnier (1998), O’Keefe (1990), Gibbs (1995) et al.

Нельзя недооценивать статус концептуальной метафоры в современной действительности. Со времен публикации Lakoff \& Johnson знаменитой и уже ставшей современной классикой книги «Метафоры, которыми мы живем» (Metaphors We Live Ву, 1980), понятие (концептуальной) метафоры чрезвычайно расширилось, изменился статус, изменилось и восприятие метафоры. Метафора приобрела репутацию понятия, которое может быть применено в повседневной жизни. Мы используем его каждый день, когда мы, к примеру, говорим или пишем. Теперь это понятие не функционирует в какой-либо одной области знания. В рамках современного научного знания метафора уже не воспринимается как вид тропа, «скрытое образное сравнение» или «переносное употребление слова» [4]. Полемика идет не только о метафорическом языке (Burke, 1945; Goodman, 1968; Locke, 1979, Stern, 2000), но также о внедрении и функционировании (концептуальной) метафоры в таких сферах деятельности человека, как политика (Tushnet, 1982, Lakoff "Moral Politics", 2002), коммуникация и журналистика (Perry J. Blackburn “The Code Model of Communication”, 2007), психология (MacCormac, 1985), юриспруденция (Suchil, 2001).

Таким образом, становится более очевидным, что изучение метафоры в рамках когнитивной лингвистики является большим преимуществом для разных областей знаний. В данном аспекте наиболее выгодно вскрывается триада язык-сознаниемышление. Изучение, протекающее в интердисциплинарном ключе, позволяет апеллировать не только понятиями когнитивной лингвистики. Изучение концептуальной метафоры является наиболее важным с учетом следующих ключевых моментов:

Во-первых, интердисциплинарное изучение наряду с когнитивной психологией, нейропсихологией, нейробиологией, психолингвистикой; искусственным разумом, компьютерной метафорой. Данная антропоцентрическая парадигма исследования 
включает в себя изучение физиологических (телесных), психических (ментальных) и психологических (эмоциональных) процессов человека. Данные процессы достаточно ярко проявляются в аспекте когнитивного тезиса о «телесной облеченности» (embodied cognition thesis). Другими словами, изучение существ и предметов действительности с точки зрения строения и ощущений человеческого тела.

Во-вторых, изучение концептуальных метафор и метафорических концептов в антропоцентрической парадигме, в аспекте «телесной облеченности» (embodied cognition thesis). Природа и образы телесности повсеместно присутствуют в нашей действительности. В процессе восприятия и концептуализации реальности, человек зачастую апеллирует именно к «телесной» составляющей окружающего мира. Так или иначе, аспект «телесности» очень часто присутствует при исследовании концептуальных метафор. Особенно ярко он представлен в эмоциональных концептах с ярко выраженным отрицательным (страх, ярость) или очень сильным положительным (эйфория, радость) элементом. Рассмотрим, к примеру, такую эмоцию как страх. По мнению психологов, страх является совершенно специфической эмоцией, которая заслуживает особо пристального внимания и исследования. Эта базовая эмоция представляет собой объект конкретных физиологических изменений, сопровождающихся экспрессивно-эмоциональной окраской. Ведущие психологи Watson (1930), Arnold (1960), Plutchik (1967), Ekman (1972), Frijda (1986) определяют страх как одну из немногих важных врожденных эмоций человека, наряду с радостью или гневом.

Концепт CTPAX/ FEAR входит в список констант любой лингвокультуры (Wierzbicka 1992, 1995). Большая исследовательская работа над эмоциональными концептами и (концептуальными) метафорами эмотивной сферы отражена в многочисленных трудах профессора лингвистики университета Eötvös Loránd в Будапеште, Zoltan Kövecses (1986, 1990, 2003). В частности, Кӧvecses отмечает, что страх (fear) можно охарактеризовать с помощью основных эмоциональных метафор, таких как FLUID IN A CONTAINER, OPPONENT IN A STRUGGLE, BURDEN, FORCE, INSANITY, а также с помощью очень специфических метафор, таких как HIDDEN ENEMY или SUPERNATURAL BEING [5].

Стимул извне (определенные обстоятельства) или внутренний стимул (переживание ситуации, которая еще не случилась, но потенциально возможно 
вызывает страх перед неопределенностью, риском, возможным отрицательным исходом) провоцирует эмоцию страха и далее ответную, зачастую защитную, реакцию человека. В данной плоскости наиболее ярко выражена связь тела, организма человека с восприятием и концептуализацией реальности.

Концепт CTPAX/ FEAR имеет обширное поле для категоризации, он очень богат семантически. Так, можно выделить некоторое количество наиболее ярких типов страха, которые практически везде, тем или иным способом, соприкасаются с понятием телесности. Разделим эти типы условно на три группы. Первая группа является базовой и несет в себе общечеловеческий страх, связанный с опасностью, (смертельной) угрозой для жизни человека, неопределенностью: «испугаться до смерти», «умирать от страха», «to scare someone to death », «to be in mortal fear of smth/smb», «on pain of death». Вторая группа включает в себя соотношение страха с каким-либо существом, предметом или зверем: «у страха глаза велики», «держать в страхе», «the eyes of fear see danger everywhere», «fear has a hundred eyes», «to keep in awe». Третья группа включает в себя страх как следствие внутренних и внешних изменений организма человека: «ноги подкосились», «дыхание перехватило», «somebody’s heart missed a bit», «to have a lump in one's throat», «to give one's the creeps».

Исследование различного рода словарей (этимологических, толковых, фразеологических, лингвострановедческих) и тезаурусов показало, что аспект «телесности» очень широко представлен как в английской, так и в русской лингвокультуре. Lakoff \& Johnson (1981) часто указывали на важную роль данного аспекта в когнитивной лингвистике при изучении концептуальных метафор и концептов, образующих их. Также представители когнитивной теории эмоций Arnold (Arnold, 1960), Lazarus \& Schachter $(1964,1970)$ отмечали значительную роль телесных изменений в Оценочной теории эмоций. Arnold писала по этому поводу: «Интуитивная оценка ситуации инициирует действие, ощущаемое как эмоция, которая выражается в телесных изменениях, и, которая может привести к неприкрытому выражению действия» [6]. В данном сегменте следует учитывать следующие моменты:

Во-первых, влияние страха на внутренние органы человека. Особенно часто встречается упоминание о сердце, ногах, а также крови и изменении дыхания: «сердце упало/ ушло в пятки», «to have one's heart in one's boots», «somebody's heart sank»; «почва ушла из-под ног», «legs go week in the knees», «legs got numb», «to feel/ to have 
the ground slipping away from under one's feet»; «fear makes somebody's blood freeze»; «язык проглотил от страха», «his tongue was swollen with fear», «with bated breath», «he was speechless». Данная группа наиболее тесным образом взаимосвязана с метонимией типа DROP IN BODY TEMPERATURE, INCREASE IN RATE OF HEARTBEAT, (see Kövecses 1990, 2003).

Во-вторых, страх сопряженный с внешними телесными изменениями человека: «мурашки по коже от страха», «быть как на иголках», «трястись как осиновый листок», «волосы встали дыбом», «бледный как стена», «to make one's flesh creep», «to give/ to feel cold shivers down one's spine», «to be on tenterhooks», «to be on pins and needles», «to shake/ tremble like an aspen leaf», «somebody's shaking with fear», «something makes somebody's hair stand on end», «as white as chalk». Данная группа состоит из большого количества метонимии типа PHYSICAL AGITATION (see Kövecses 1990, 2003), а также MOVING AGAINST, TENSION (see Davitz 1969).

В-третьих, влияние страха на разум человека: «потерять рассудок», «обезуметь от страха», «душа в пятки ушла», «to go out of one's mind because of fear», «to lose one's reason», «his mind was blank», «to frighten/ scare living daylight out of someone». Данная группа определяется большим количеством метонимии типа INADEQUACY, SLUGGISHNESS, SLOW MOTION/ DELAY MOTION, (THOUGHT) OBSTRACTION.

Существует некоторое количество подходов к изучению составляющих концептуальной метафоры, i.e. ключевых концептов внутри метафоры. Цель их определена важностью глубокого понимания природы проявления и функционирования ключевых концептов и самой коцептуальной метафоры. В данной области важным являются системы категоризации и кодирования. При изучении концептуальных метафор с эмоциональной составляющей в легальном дискурсе важным является, к примеру, определение степени влияния эмоциональных концептов с целью психологического воздействия (е.g., манипуляции, убеждения, принуждения), которое может привести к ментальной оценке принятия/ изменения решения у присяжных или судьи. Данные задачи успешно решает набирающий особую популярность в настоящее время квалитативный (качественный) анализ.

Квалитативный (качественный) анализ исследует текст в его неформализованном виде. Последнее десятилетие показало рост такого рода исследований, также программное обеспечение, поддерживающее квалитативный 
анализ, вышло на более качественный уровень (Weitzman \& Miles 1995; Feldman 1994; Coffeez, Holbrook \& Atkinson 1996; Thomas Muhr 1993; Gibbs 2002 et al). Квалитативные методы исследования, также известные как интерпретативные, более сложные для компьютерной обработки. Процесс восприятия, понимания и интерпретации информации - это многогранный по своей природе механизм. На компьютерном рынке лидирующие позиции в основном ранее занимало программное обеспечение для количественного (корпусного) анализа. Специалисты в области технологий для социологических исследований, Fielding \& Lee справедливо отметили, что у научного мира ушло достаточно много времени на достижение сегодняшнего уровня программного обеспечения, которое используется для качественного анализа (Qualitative Data Analysis) [7]. Сегодня такие комплексные программы как NUD*IST, NVivo, Atlas.ti или MaxQDA поднимают квалитативный анализ на более высокий уровень.

Понятиям страха, устрашения, запугивания отводится довольно большое место в художественной литературе с криминалистичекой и юридической тематикой, как в классической (Agatha Christie, Arthur Conan Doyle, Harper Lee), так и в современной (John Grisham, Michael Connelly, William Bernhardt). Рассмотрим отрывок из художественного текста, а именно, юридического триллера американского писателя John Grisham “The Last Juror” (2004). Некоторые исследовательские вопросы в данной тематике могут быть направлены на определение, к примеру, следующего: (а) какие психологические и тактические приемы наиболее часто используются адвокатом в ходе судебного процесса; (b) какова степень психологического влияния данных приемов на людей в зале суда; (c) какие группы фраз невербального выражения состояния человека (выражение лица, жесты еtс.) наиболее часто встречаются в тексте, описывающем процесс суда присяжных.

В ходе квалитативного анализа и аналитического кодирования текстовых ситуаций, происходивших непосредственно в зале суда, выяснилось, что эмоции и их воздействие на людей в ходе процесса весьма высоки. Нужно заметить, что ассоциативно-семантическое поле legal trial/ court trial довольно ярко представлено концептуальной метафорой LEGAL TRIAL IS WAR. Данная группа включает в себя ряд концептов, тесно связанных со следующими понятиями: argument, fight, battlefield, war. Обратим большее внимание на группы, связанные с проявлением тех или иных эмоциональных всплесков. Так, были выявлены наиболее часто встречающиеся 
эмоционально окрашенные группы и подгруппы, в том числе: 1) Lawyer tactics: general psychological manipulation, persuasion, intimidation; 2) Perception influence and attitude change: persuasion and social influence (позитивное и негативное влияние), и perception tricks to change attitude (чаще негативное влияние).

К примеру, стратегическая тактика адвоката особенно широко представлена группой intimidation с разной степенью градации устрашения (e.g., permissible intimidation, force intimidation), нацеленная на пробуждение таких эмоций, как страх, ужас, чувства беспокойства и тревоги: “When Ernie finished we all felt as though we'd been tampered with. Anyone who'd talked about the case, which of course was every person in the country, seemed in danger of being indicted by Ernie and hounded to the grave" [8].

Процесс восприятия (perception) всегда связан со способностью быть осведомленным о чем-либо, давать оценку чему-либо с помощью и через (а) сознание и (b) чувственное восприятие с элементами интуиции [9]. Восприятие тесным образом связано с эмоциональными импульсами (Forgas 1991; Goleman 1995; Niedenthal, Halberstandt, Margolin et al. 2000, Ekman 2009). В следующем примере, оценка при восприятии в большой степени регулируется сильными негативными эмоциями (ненависть, отвращение, омерзение):

“Then, there were photographs of Rhoda's corpse, a series of eight by ten's that were handed to the jurors and passed around. Their reactions were amazing. Every face was shocked. Some winced. A few mouths flew open. Miss Callie closed her eyes and appeared to pray. Another lady on the jury, Mrs. Barbara Baldwin, gasped at first sight and turned away. Then she looked at Danny Padgitt as if she could shoot him at point black range "Oh my God," one of the men mumbled. Another covered his mouth as if he might throw up" [10].

В данном случае срабатывает стандартная схема порождения эмоции по когнитивно-физиологической теории Schachter (1970), где происходит когнитивная оценка события и когнитивная оценка состояния организма, что приводит в конечном итоге к эмоциям страха, гнева, состояниям шока, беспокойства, к чувствам отвращения, антипатии и тревоги.

Группа perception наиболее ярко представлена подгруппой perception trick to change attitude: 
"Danny was nicely but casually dressed in a white long-sleeved shirt and a pair of starched khakis. As instructed by Wilbanks, he was smiling a lot, as if he were really a nice kid whose innocence was about to be revealed" [11].

Опрятно одетый, приветливый человек скорее вызовет положительные ассоциации и доброжелательное к себе отношение, нежели неприязнь или отвращение (pattern manipulation). Такое намеренное психологическое манипулирование направлено на маскировку истиной личности подсудимого (wolf in sheep's clothing) и попытку извлечь положительные эмоциональные импульсы у присяжных, судьи и присутствующих в зале суда.

Список концептуальных метафор постоянно расширяется. Однако необходимо заметить, что метафор посвященных концептуальной связке Law and Emotions попрежнему остается недостаточное количество. Причиной этому может послужить то, что соприкосновение, и особенно взаимопроникновение сферы закона и сферы эмоций не было достаточно изучено в симбиозе. Понятия здравого смысла до какой-то степени позиционировалось отдельно от эмоций, чувств и настроений человека. Когнитивный аспект исследования эмоциональных концептов в легальном дискурсе с применением квалитативного анализа позволит более детально оценить спепень вовлеченности эмоций в тексте (художественном, публицистическом, юридическом). Также он позволит оценить степень влияния эмоций на умственные процессы, применяемые в легальной практике, а именно процессы оценки событий и принятия/ непринятия решений. Данные, собранные и обработанные в ходе анализа могут применяться при составлении или спецификации концептуальных метафор.

\section{Список литературы}

[1] Маслова В.А. Когнитивная лингвистика / В.А. Маслова.- Минск.: ТетраСистемс, 2004. - c. 6

[2] Красных В.В. Этнопсихолингвистика и лингвокультурология / В.В. Красных.- М.: Гнозис, 2003. - с. 35

[3] Boyle, Robert R, S.J. (1954) The Nature of Metaphor, The Modern Schoolman 31, p. 257 
[4] Ожегов С.И. (2003), Толковый словарь русского языка: 80000 слов и фразеологических выражений/ Российская академия наук. Институт русского языка имени В.В. Виноградова/ С.И. Ожегов, Н.Ю. Шведова. - 4-е. изд., дополненное. - М.: ООО ИТИ Технологии, с. 353

[5] Kövecses, Z. (2003) Metaphor and Emotion. Language, Culture, and Body in Human Feeling, Cambridge University Press, p. 23

[6] Arnold, M.B. (1960) Emotion and Personality, New York: Columbia University Press, p. 177

[7] Fielding, N., Lee, R. M. (1998) Computer Analysis and Qualitative Research, SAGE Publications, p. 11

[8] Grisham, J. (2004) The Last Juror, Doubleday; $1^{\text {st }}$ Ed., p. 95

[9] American Psychological Association (APA): perception. (n.d.). Collins English Dictionary

- Complete \& Unabridged 10th Edition. Retrieved August 13, 2012, from Dictionary.com website: http://dictionary.reference.com/browse/perception

[10] Grisham, J. (2004) The Last Juror, Doubleday; $1^{\text {st }}$ Ed., p. 100

[11] ibid. [10] p. 93 


\title{
Semantic development of preschool children
}

\author{
Baisheva M.I. \\ «North-Eastern Federal university named after M.K.Ammosov»
}

\begin{abstract}
The purpose of the article is updating of semantic forming approach to development of personality in the spiritual moral sphere. The main topic of this article is organization of semantic forming context of education in pre-school childhood. The author pays attention to the semantic forming essence of people's culture of education and takes necessary the inclusion of spiritual moral reading of oral folklore by children in the educational system, sensitivity and pedagogical conditions of semantic forming activities of pre-school children is justified.

Keywords: meaningful education, spiritual and moral development, traditional culture, folklore, independence, pre-school age.
\end{abstract}

All cataclysms occurring in spiritually-moral sphere of a modern society, confirm inevitability of transition to a personal-semantic paradigm of education. Probably, today it is necessary to staticize and comprehend all arsenal of ideas, methods, technologies of semantic formation and developments of intrinsic bases of the person, proved in domestic pedagogics for creation of real dynamic conditions of prompting of the person to self-development and self-creation.

Semantic formation is the most effective at integration of potential of modern education with Cultural creating and human creating essence of traditional culture of education. In the course of the development the traditional culture of education of children in Russia was issued as the greatest spiritually-moral value of the people.

At the time of K.D.Ushinsky in reading book of an initial course of domestic literature there was a section of the spiritually-moral reading, which main objective - education of love to native sources. On K.D.Ushinsky's deep belief, education appointment - to grow up the person having a specific goal in life. The analysis of works of K.D.Ushinsky allows to conclude that one of the greatest means Semantic forming developments of the person is the people folklore.

Considering that oral national creativity as the ethnopedagogical phenomenon and a source of national values demands more substantial approaches to studying and a reflection of values, we have made an attempt to revive the forgotten tradition of domestic literature and 
have solved in modern quality to rethink process of "spiritually-moral reading» children in educational institutions of our republic.

In a context of a humanization of pedagogical process essentially important positions of model of "spiritually-moral reading» were: not assimilation and not accommodation, and integration into persons original national and universal as comprehension of own cultural advantage and advantage of others, an openness and creativity to the world; support of subject-matter of the child at all stages of activity, his Semantic forming activity in selfdevelopment and Destiny building, development of universal infinity, «an exit for limits of», openness to the world; Maintenance of Cultural conformity of education and development of the child when cultural values act: on the one hand, as set of achievements of a society, with another - as a source of creative transformation of the person; in this context the understanding of the valuable maintenance of folklore is considered by the child as a reference point of creating life and mastering by values in own «a reflective key» (V.M.Rozin); the model end result is an emotionally-moral experience of values of a fairy tale, bylinas, legends, legends etc. by the child,: understanding of his participation in destiny of a family, the people, mankind; destiny of native places, the native land and comprehension within the possibilities of own responsibility and at the same time the security in each of them;

Spiritually-moral reading of national literature, on the one hand, staticizes an openness of the child to the world, his valuable-semantic relation to him, and with another, urges on parents, teachers to Semantic forming development of the person of children. Hence, the spiritually-humanistic person can be developed only on the basis of amplification of ways, ascension ways to values-ideals and actualization of an is subjective-personal component of value. Ignoring of an is subjective-personal component of intrinsic bases of the person leads to a spiritual impoverishment.

Special sensuality is shown in the process of measurement, an active projection, interpretation of values, identification of with the creating person at the child. He finds out own uniqueness, connectivity, responsibility to destiny own both parents; the maintenance and technology of work with children provide developing influence of values on consciousness of children and promote psycho-emotional satisfaction, development of steady emotionally-valuable displays, creative-informative activity, humane style of behavior. 
The preschool age is the sensitive period for formation of spiritually-humanistic bases of the person, comprehension of own "connectivity" (N.E.Shchurkova) with destiny of a family, the native people and as a whole mankind.

In researches of domestic psychologists it is established, what exactly ability and requirement to operate at preschool age at children for the first time are shown, being guided by «internal ethical instances». On L.S. Vygotsky, this reference point grows out of mastering of moral requirements of a society. Occurrence of internal ethical instances testifies that external requirements turn in internal, which kids carry out voluntarily, irrespective of control of adults show them to another and to himself.

In a context of a studied problem V.A.Petrovsky, S.L.Rubinshtejna, V.E.Frankla's views are close to us, etc., confirming about infinity of the person. V.E.Frankla's statement: «to be the person - means to fall outside the limits itself. I would tell that the essence of human existence consists in his transcendo» speaks about necessity of enrichment of some approaches to studying of intrinsic properties of the person, allowing to be a social and spiritual being.

Creation of pedagogical conditions of Semantic forming formations of children of preschool age should correspond to age of the child, subculture of the childhood and to be based on following ideas:

- Development of intrinsic valuable potential of the child and if isn't present by the end of preschool age it has quite often an effect (M.I.Lisina, N.I.not, S.L.Rubinshtejn, etc.) should become a leading line of development of the person till the end of preschool age;

- The major aspect of sense of the childhood in human culture consists in opening himself and another as person, in finding of a measure of human in the person (социокультурном, spiritual formation) that underlies development of individual; children in 5-6 years (62 \% of children) prefer conversations on personal themes in dialogue with adults, i.e. «in process of a growing preschool children pass to contacts of the profound moralpersonal plan» (Z.M.Boguslavskaya, A.G.Ruzskaya, E.O.Smirnova, etc.);

- Dialogical interaction is natural quality of the child that dialogue along with game is «this element in which lives and develops the child» (F.Frebel); the organization of philosophic conversation, under N.E.Shchurkova's statement, this education of ability to 
generalizations that the child could see the life phenomena behind the fact, behind the phenomenon - laws, and behind laws to distinguish bases of human life »;

It is necessary to underline that today educational activity in educational establishments, should be considered as construction process of semantic vital concepts of the person and an ascension in "spiritual- I". Thus the education system which is institute of the state, responsible for formation and development of the person, should be improved, being supplemented with the innovative maintenance and being enriched with the technology, focusing on semantic formation.

\section{References}

1. Рерих, Н. К. О Вечном / Н. К. Рерих. - М. : Политиздат, 1991.

2. Полозова, Т. Д. Не склад исторической продукции, а совершенно живое явление / Т. Д. Полозова // Педагогика. - М., 2002. - № 2.

3. Франкл, В. Э. Человек в поисках смысла / В. Э. Франкл. - М. : Прогресс, 1990.

4. Венгер, Л. А. Проблемы формирования познавательных способностей в дошкольном возрасте / Л. А. Венгер. - М. : Знание, 1980.

5. Лисина, М. И. Общение, личность и психика ребенка / Под ред. А. Г. Рузской. М. : Изд-во Ин-та практ. психологии; Воронеж: НПО МОДЭК, 1997. 


\title{
Ecological education of pre-school children in developing environment
}

\author{
Lyubov Andreeva, $\mathrm{PhD}$ \\ Pedagogical Institute, the North-Eastern Federal University, the Republic of Sakha \\ (Yakutia), Russia
}

\begin{abstract}
The relevance of environmental protection requires thinking how a nearby nature area helps to raise ecological awareness of the child, because in different seasons the nature can affect different chord in the hearts of children. It is pedagogically appropriate to organize the rich developing environment for educating of the child to love his mother nature. The problem of using ecological path in educating preschoolers for love their mother nature is very important as the process of the child's interaction with nature is contradictory. The purpose of its creation is teaching children on the examples of specific natural objects, the contact with nature, nurture relations, caring for it. Attaching the child to the world of local nature, we consciously develop different aspects of his personality, arouse his interest and desire to learn his native natural environment, cause the child to severe sympathy to living animals, show the uniqueness of life in any, even the most bizarre form.
\end{abstract}

The relevance of environmental protection requires to think how a nearby nature area helps to raise ecological awareness of the child, because in different seasons the nature can affect different chord in the hearts of children. E.Y. Alexandrov said that a special role in the process of familiarizing preschoolers for their land was given to the multiplication of the child's contact with other people. Through these contacts, the children begin to perceive and evaluate themselves and others in carrying out the conversion of the motivational sphere of other people. So patriotic feelings raise, the child adapts to the social environment.

It is pedagogically appropriate to organize the rich developing environment for educating of the child to love his mother nature. Learning Environment in the Preschool Educational Institution (PEI) is a space of life for children of all ages.

Organizing activity of the child, you need to give him support in his needs. The task of the teacher is to guide his interest to the right direction. Formation of children's representations about the changes in the nature requires constant and direct contact with it. For example, in the PEI "Kencheeri" it was created a special condition: the creation of an ecological trail. Nature trail is a variety of educational nature trails. The problem of using ecological path in educating preschoolers for love their mother nature is very important as the process of the child's interaction with nature is contradictory. The purpose of its creation is 
teaching children on the examples of specific natural objects, the contact with nature, nurture relations, caring for it. Attaching the child to the world of local nature, we consciously develop different aspects of his personality, arouse his interest and desire to learn his native natural environment, cause the child to severe sympathy to living animals, his desire to help, show the uniqueness of life in any, even the most bizarre form.

An initial link of educating consciously correct attitude of preschoolers to nature is a system of specific knowledge, reflecting the major laws of nature: the variety of species, their adaptation to the environment, changes in the growth and development of life in the communities. The specificity of the knowledge system is its design in a specific, limited material, which is available for the observation of children, the knowledge through visualfigurative thinking. These provisions are criterial for developing of ecological environment in the PEI. Proper organization of the zone of nature involves assimilation of an environmental approach to the life of plants and animals and especially methods of environmental education of children by workers of pre-school institutions.

Ecological environment in the kindergarten - concrete animals and plants that live permanently in an institution and are under the care of adults and children. It is very important that teachers and other staff of the kindergarten knew ecological features of each object of nature - its need for these or other factors of the environment, the conditions in which it is doing well and growing.

Strict adherence to an environmental approach to the equipment of an area in kindergartens will allow children to see:

1) the most common and indissoluble bond of the living organism and the environment;

2) morphofunctional adaptation to specific elements of habitat;

3) the birth of a new organism, its growth, development and facilities, providing these processes;

4) the specificity of the living organism (plant or animal), it difference from other subjects;

5) the variety of living organisms, and different ways of interacting with the environment. 
Nurture love for mother nature in children of the age of 3-4 - a means of formation in their minds a realistic knowledge of the natural environment, based on sense experience and upbringing of proper relationship to it. The interaction process of a little child with plants and animals is contradictory. Individual attitude to the nature may occur in children a moral and an immoral action. Consciously correct attitude is produced when there is close contact and various forms of the child's interaction with plants and animals, that are in the room and in the area of $\square$ kindergarten [3, c. 33].

The basic principles of arrangement and equipment of a developing environment in preschool institutions are: age, group and individual isolation, creating comfortable light conditions, optimal air-heating systems, providing sufficient physical activity, creating conditions that are close to natural.

Children adjust to the perception of something unusual, the appearance of the environmental room is very different from all others, that they see every day. In some kindergartens it was indicated that adaptation of newly arrived children passes quickly, if they like unusual conditions [2, c. 33].

As an important element the nature trail is included in the developing environment of PEI, directed to the upbringing of children's love to their mother nature.

Often children do not see what trees grow in the area of their kindergarten, on their own street. Systematic activities and observations of nature of the kindergarten help to ensure that children immediately recognize the familiar flowers, shrubs and trees. For educating of love for mother nature it is necessary to organize an environmental activity. The Environmental activity in preschool educational institution helps to ensure that the process of becoming consciously correct attitude to nature is accompanied by certain behaviors of a child, which can serve as a criterion to assess the level of environmental education. These are independent observations, experimentations, the issues, the desire to talk about feelings and experiences, to discuss them, to embody in a different activity (to reflect in a game, to create an art-production, to care for animals and plants) [4, p. 34].

Children of preschool age have "nature-loved instincts", that with appropriate leadership can be developed into stable personal qualities, stable patriotic feelings. In objects of an ecological trail in the kindergarten great attention is paid to the specific natural phenomena, weather observations, the right skills of ecological culture. Equipping the 
territory of the kindergarten, we should remember that the nature trail is not a flowerbed that should be completely tidy, decorated with flowers, but a part of nature close to the child, preserved or carefully recreated by people.

Children's understanding of natural phenomena in animate and inanimate nature should grow and multiply. At this time a realistic understanding of these phenomena and the ability to establish relationships between them are formed. Perception of nature helps to develop emotional, sensitive, caring attitude towards all living beings.

In the younger group, the developing environment as a nature trail was organized for educating of love for mother nature. Emotional responsiveness to the effects of the natural environment is formed by the children in such conditions. Children, learning in this environment, are able to do useful environmental work, they develop control over their actions.

Preschool children educator should know the features of this age in his work on the aesthetic education with children. Children of this age have a great desire for autonomy and independence. They want to see everything, to open everything themselves.

In the realization of the tasks of upbringing love for mother nature great importance has the natural environment in the kindergarten. In the kindergarten "Kencheeri" they are nature corners in all groups, a nature room, a winter garden, a correctly cultivated land, which provide an opportunity to contact with nature, the organization of systematic observations of natural phenomena and objects, the familiarizing children to regular labor.

In the kindergarten a special nature trail was created, a natural area with wild plants. Recommendations of the program "Our home is nature" by N.A. Ryzhov were used for the development of the ecological trail.

According to the existing recommendations, nature trail is to be connected in such a way that it crosses as many different types of landscapes, for example, a riverside, a hill, a lined meadow, etc.

Creating the environmental trail in the kindergarten "Kencheeri" began with organizational work for production of a map of zoning area. Fragments of natural plant communities, typical especially for this place, were formed on the trail. Forest, steppe, meadow corners were equipped on the plots, where children can learn about the local flora, fauna and ecosystems of meadows and forests. The more diverse the plants on the ecological 
path are, the more children have the representations, associated with certain plants, conditions of their nutrition and habitat.

Before you allocate a space for the planting of different plant societies, teachers have to explore the territory of the yard, highlighting the most shaded and areas, wetter and drier ones. It was determined this way, where are the best conditions for steppe plants (open dry sites), forest plants (shaded, moistened) etc. If possible, it is necessary to create a small pond with aquatic plants, which has a positive effect from cognitive and aesthetic points of view.

Trees of different ages, including one withered tree were presented on the ecological path. It allowed us to introduce children to a number of insects, lichens, fungus, mosses, which are usually settled on these trees, and to explain the cycling of matter in nature. For this purpose it was necessary to include in the path a stump and an old fallen tree, covered with moss and lichen. That tree was brought from the nearest forest.

Diverse life forms of plants: trees, shrubs and grass were presented on the ecological path of the kindergarten. Beautiful flowering plants were selected in such a way that within a year one flower replaces the other, so we created a garden of continuous flowering. There was a small group of weed plants, which did not interfere with other plants to grow, and it was no need to destroy it. It also became a part of the trail.

On the example of weeds we explain children many biological characteristics of plants, the human impact on vegetation societies. With the same purpose we left small trampled areas on the path. Comparing them with others, we clearly showed the children how the vegetation cover changes under the influence of trampling. We sought to make objects of the environmental trail various. There is no need to have all of the trail looked perfect: to clean dry autumn leaves, to uproot old trees, to destroy completely all the weeds.

During walks and excursions on the ecological path we introduced children to plant and animal life, gave the knowledge about some insects and animals of those places, where their kindergarten is situated. For example, in order to children understand the harm that leafeating caterpillars bring to plants they were placed in a fish tank with the plant on which they were found. After watching it could be seen as caterpillars strike leaves in a short term. In the conversation the teacher underlined the benefit of birds, destroying these caterpillars. Considering that at present there are very few butterflies, children didn't catch them and put into the cages, they watched them on walks, flying or sitting on the plants and grass. 
Moreover, we didn't allow children to collect a large number of insects, that children sometimes like to do, they could catch 1-2 species of insect, watch them in cages for a few days and release, explained what benefits they bring to nature.

Younger preschoolers also were developed their educational interest - they examined plants, participated in the collective observations, made sketches in their calendars, asked questions, listened to stories of the educator. We developed aesthetic sense of children, the ability to notice and assess attractive qualities of plants: the beauty of shape and color of leaves, vines, flowering plants. Children noticed and react emotionally on emerging shoots, buds, flowers.

Through training on the ecological trail we developed the perception of plants as living beings: children could see their poor condition (drying up leaves, pale color, elongated stems, etc.); detect failure conditions for their life (dry land, low light). Children sympathized plants: they reported on the observed failures, fulfilled instructions on plant care, helped in their transplantation.

Children got acquainted with certain types of trees, shrubs, cultivated and wild plants, garden and kitchen plants. They learned their names, features, structural features (roots, stems, branches, leaves, flowers, seeds) and the appointment of all organs. They also learned that all plants are creatures, that for life, growth, maturation of seeds they must maintain their integrity, they need certain conditions (heat, light, moisture, soil nutrients, air). These conditions are at the site of the kindergarten, so the plants grow there. The weather conditions are not constant, they are seasonal. Plants have adapted to life in the changing conditions.

The most favorable conditions for plants of ecological trails are in summer: it is warm, hot, days are long, a lot of sunshine, rains, loose soil absorbs rainwater, it nourishes roots of the plants. At this time, there is a lot of grass, flowers, green foliage on trees and shrubs, all plants have a stormy life - they grow, flower and produce seeds.

In autumn environmental conditions of the path change: it is getting cold, less light (days are shorter). Winter conditions of the environmental trail are unfavorable: it is very cold, little light, snow. In spring the conditions change again, it is getting warmer and warmer, days become longer. The plants come to life, a new life begins.

In the educating children to love for mother nature we used objective, plot and art paintings, photographs. Viewing paintings should be done repeatedly, as it is necessary to 
provide the perception of the individual parts of the picture, and the whole image, art paintings, landscapes are essential for the development of aesthetic perception of nature and feelings of children in relation to it. We plan to work out the "Green Book". Children will collect materials about the nature of Yakutia, essays, short stories, poems, newspaper clippings and photographs. For this book we collect puzzles, invented by children, fairy tales and stories, which are based on knowledge of nature.

Thus, the creation of developing environment in kindergarten promotes educating children to love for their mother nature. Upbringing a child through his love for his mother nature will allow to form right attitude to nature, will teach the rules of interaction with its objects. Conscious relationship with nature can be formed in preschoolers in the conditions of close contact and various forms of the child's interaction with plants and animals.

\section{References:}

1. Dmitriev, Y.D. About nature to adults and children. - Education, Moscow, 2002. - 244 p.

2. Kondratyeva, N. Program of environmental education for children. "We are" - The Union, St. Petersburg, 2006. - 214 p.

3. Nikolaeva, S. Education of an environmental culture in the preschool childhood. - The New School, Moscow, 2005. - 160 p.

4. Ryzhov, N.A. Leadership of the teacher to educate children with nature / / Preschool. 1991. - № 7. - P. 34.

5. The system of patriotic education in the PEI / Comp. E.Y. Alexandrova and others Teacher, Volgograd, 2007. - 203 p. 


\title{
Tolerance formation in the course of intercultural communication
}

\author{
Nikolaeva L.V., \\ Master of pedagogical sciences, Yakutsk
}

\begin{abstract}
In the article we reveal the ideas of the pedagogy of tolerance that should be transmitted through education and cultural policy. We also reveal the theoretical bases of tolerance formation of a personality in the context of globalization, criteria of individual's tolerance and conditions of its formation. Basis for the formation of individual's tolerance is learning to intercultural communication. We consider the definition of the theory of intercultural communication. Linguistic cultural approach is aimed to create an evaluative attitude to language as a social phenomenon, as a means of reflecting national and universal in the cultures of studied languages as a means of intercultural communication. It is necessary to educate basis of tolerance from pre-school age children, form tolerance to each other, understanding, interest and respect for the traditions of their own and other peoples, introduction to general cultural values, features of communication and culture of the peoples of other countries, education of friendly relations and training to intercultural communication.
\end{abstract}

Tolerance is a necessary and fundamental value for realization of human rights and an achievement of peace. In the simplest and main form tolerance is recognition behind others of the right to respect of their personality and the self-identity, formed a basis for today's international standards of human rights.

In the modern conditions, rates which were characterized by increase in globalization and integration of all vital processes and the phenomena, on the one hand, and aspiration to isolation of national cultures, with another, - special value is gained by a problem of tolerant relationship of representatives of various cultural communities. The perception of cultural diversity and cultural distinctions conducts to understanding of need of the dialogue equal in rights which main objective is ensuring humane relationship of representatives of different national cultures, acceptance of principles of tolerance and cultural pluralism.

Scientists of the world, UNESCO documents clearly express idea of need of tolerance for society, which can't be tolerant to intolerance, and the hostility caused by wars and the interethnic conflicts. Tolerance recognition as a world condition between the people, human rights is the main task of democracy.

Implementation of these values in the world community will make the basis «cultures of the world». Any culture in essence grows out of training. Education is training, at the heart of which and in which implementation cultural values lie. At the heart of education of culture of the world values of the world, human rights, democracies and, the most important, tolerances lie.

The special sphere of the theory and practice of tolerance in the modern world - is intercultural communication. 
By Furmanova V.P. definition intercultural communication represents dialogue of cultures. Intercultural communication as dialogue of cultures is way of universal communication which covers exchange of information and cultural values in a context of interethnic communication [9, page 38]. The main objective of intercultural communication at the present stage of development of society consists in making active humanitarian potential, to aim it at development and the approval of the concept of a priority of universal values, rapprochement of cultures of the different countries by introduction in consciousness of pupils of feeling of unity and value of our civilization.

Intercultural communication as dialogue of cultures - the multidimensional phenomenon representing now interest and philosophers, both linguists, and psychologists, and лингводидактов, and methodologists. In its contents interaction is traced: a) the language reflecting culture of the people; б) the culture transferring an originality of socio-historical conditions; в) the personality formed during practical activities.

Intercultural communication is considered as communication process (verbal and nonverbal) between the communicant being carriers of different languages and cultures or set of specific processes of interaction of people, belonging to different cultures and languages (Haleeva I.I.). In the conditions of intercultural interaction between communicating there are intercultural relations. Participants of communication, making use of the linguo-cultural experience both the national and cultural traditions and habits, at the same time try to consider as well other language code, other customs, other traditions, other norms of social behavior. If it doesn't occur, the act of intercultural interaction can't take place or be broken because of emergence so-called «communicative failures», having more serious negative consequences for mutual understanding of partners in communication, than language mistakes.

The socio-linguistical aspect of studying of intercultural communication is based on the concept of universality of the communication processes occurring at level of interpersonal, inter-group and international communication. The insufficient attention to existing sociocultural distinctions between contacting groups and native speakers influences extent of mutual understanding and trust level. Theoretical problems of intercultural communication and communication are investigated in works M.M.Bakhtina, V.S.Bibler, V.V.Vorobyev, M.S.Kagan, R. Lado, Safonova's Accusative, In the masculine Singer, E.I.Passova, Furmanova, I.I.Khaleeva, G.A.Zuckerman's Accusative, etc.

Now, when the new relation to international relations is formed, there is an exchange of cultural values at all levels: international, national and individual. Just as psychoanalysts will improve theories of communications more completely to describe nature of interaction between the patient and the doctor, and practice of the international relations needs systematic immersion in particular this or that culture to find a key to more effective intercultural communications (Dodd). In this plan the new directions in language pedagogics are the cultural sociality of education (Safonov V.P.) and research on a lingual cultural science 
(Vorobyov V. V.). The cultural sociality of the content of education by Safonova V.P. definition is understood as strengthening of functions of educational systems as transmission media the pupil of universal and national cultural values on the basis of comparative studying of languages and cultures, their influence at each other, complementarity in world process of universal culture. «Lingual cultural science - the modern humanitarian discipline of synthesizing type studying interrelation and interaction of language and culture in its functioning and reflecting this process as complete structure of units in unity of their language and extra language (cultural) contents by means of system methods and with orientation to modern priorities and cultural establishments (system of norms and universal values) [5, page 36]. Lingual cultural science competence means not only knowledge of value of concepts and words, but also all system of the cultural values expressed in language.

The Lingual cultural science's approach is directed on formation at trainees of the valuable relation to language as to the social phenomenon, as to means of reflection of the national and universal people, as to means of intercultural communication, generalizations of knowledge of perception of the world by various ethnoses, the people in their spiritual, material culture and its various forms of existence.

Lingual cultural science arose on a joint of such interdisciplinary sciences, as a Linuval regional geography, ethnolinguistics, ethnoetiquette, social linguistics, communication psychology, cultural anthropology, history of world and national cultures which help to carry out valuable and orientation studying of cultural identity of the people and their contribution to development of world culture and understanding of as the subject of culture capable to intercultural communication.

Tolerance is formed on the basis of development of ability and readiness to understand mentality of representatives of other cultures. Numerous examples testify that the people rather well knowing a foreign language, having appeared in the environment foreign, very often meet difficulties because of ignorance of cultural distinctions and sociocultural standards of behavior in this country. In mentality 3 components forming "a mentality triangle" are interconnected:

\section{Knowledge}

\section{Relations Behavior}

The concept "mentalities" calls conditional line behind which there is possible or impossible a proper response.

At communication of representatives of different cultures there is some threshold preventing adequately to perceive behavior of each other, i.e. the cultural interference is observed. According to R. Lado, the culture is the structured system of simulated behavior for native speakers which doesn't extend the action through borders of cultures [4, page 50]. 
Cases when the bad knowledge or a complete ignorance of culture, customs and traditions of the countries - diplomatic partners of Russia led to considerable interstate friction are in the history known. The knowledge of customs and culture plays not less important role, than knowledge of language.

\title{
Cultural iceberg
}

\author{
food \\ clothes \\ dwelling \\ living conditions \\ knowledge (education) \\ behavior (habit) \\ belief \\ ability of perception
}

The model of culture of R. Gibson is of interest for our research. Robert Gibson represents culture as a cultural iceberg in which the main aspects of life and customs, habits and laws of each people are concentrated.

If to present formation of a cultural background of knowledge as the top part of an iceberg, it is clear that for effective intercultural ties it is important to see and understand the lower part of an iceberg: behavior, belief, perception, character, i.e. mentality of the people.

Each people have features of communication: sociability, an outlook, self-expression, the relation to public opinion, social symbolism, national etiquette, symbolics of gifts, colors, speech etiquette, distance at communication.

Robert Lado in the work «Linguistics over borders of cultures» emphasized that training to another's culture should be carried out through comparison to the culture. On R.Lado of unit of the simulated behavior, inherent in each culture, have a form, value, the distribution which comparison can help with overcoming of difficulties of understanding of another's culture. For example, same form, different value. It gives an example traditional bullfight in Spain which was always a source of intercultural misunderstanding.

Form. Bullfight has very exact and difficult form. The person armed with a sword and a red raincoat, throws down to challenge a bull and kills him in fight. The matador, a bull, picadors, music, clothes are a part of a form. 
Value. Bullfight transfers a complex of values in the Spanish culture. It is a sport. It symbolizes triumph of art of the person over a brute force of a bull. It is courage demonstration.

Distribution. Bullfight is characterized by difficult distributive model. There is a season of bullfights in an annual cycle, favorite days in a week cycle, favorite time in a day cycle. Bullfight occurs on the special arena and in the special place, known to any person in this culture.

Form, value and distribution by eyes of the representative of other culture. The American viewer will see a form: the man in special clothes, with a sword and a red raincoat in the hands, provoking and killing bull. For him it is a murder of a "defenseless" animal by the armed person. It is dishonest, it isn't sports, cruelly.

Such opposite perception speaks different concepts - a bull and the person - in two cultures. In the Spanish culture of people it is not considered physically strong, but it is dexterous and clever. The bull strong, but isn't reasonable. In the American culture the people are physically strong, a bull too. Feelings of pain, grief, pity etc. (stories about animals, among them «the Bull Ferdinand») are also inherent in a bull. The bull deserves equal chances in fight.

Thus, mastering by culture of other people assumes assimilation not only corresponding «the culture facts», but also their specific national mentality that will help to expect possible misunderstanding between representatives of different cultures, to prevent complications in relationship and, keeping national originality, to take steps towards each other. The Swedish researcher K.Yonsson notes that usually at coincidence of interests of the parties, at cooperation national distinctions aren't noticed, but it is necessary to arise to the conflict as they start to play important role.

In the tideway of our research we allocate four main components of tolerance is a recognition, acceptance, respect and understanding. Between people today it is possible to consider acceptance and recognition of distinctions as the most acceptable form of intercultural interaction. In the United Nations accepted in 1995 concerning education, a science and culture it is specified «Declarations of principles of tolerance»: « tolerance means respect, acceptance and understanding of rich variety of cultures of our world, our forms of self-expression and ways of manifestation of human identity. Tolerance is a harmony in variety. This concept meaning refusal of dogmatism, from absolution of truth and approving the norms established in the international legal acts in the field of human rights» [3, page 44]. Original tolerance is valuable moral quality of the developed personality, i.e. the personality which has the values and interests and is ready, if it is required, them to protect, but at the same time yours faithfully treats views and values of other people. Tolerance assumes 
understanding of that the world and the social environment are multidimensional, so, views of this world are diverse.

At the same time tolerance demands natural, healthy hostility to the negative phenomena in respect of ideology, communication and behavior.

It is possible to allocate the following criteria of tolerance:

- equality (equal access to the social benefits, to administrative, educational and economic possibilities for all people, irrespective of their floor, race, a nationality, religion etc.);

- mutual respect, benevolent and tolerant relation to various groups (to disabled people, refugees etc.);

- preservation and development of cultural originality and languages of ethnic minorities;

- coverage by events of public character, holidays as it is possible bigger number of people if it doesn't contradict their cultural traditions;

- possibility to follow the traditions for all cultures presented in society;

- freedom of a religious belief provided that it doesn't strike at the right and possibility of other members of society;

- cooperation and solidarity;

Thus, the modern concept of essence of tolerance is based on recognition, acceptance and respect of dissimilarity, distinction, readiness for dialogue, for intercultural communication. It is thus important to form ethno-cultural competence, which assumes only objective information on other people at younger generation. Ethno-cultural competence is understood «as process of acquisition by the individual of objective knowledge and experience in area of ethnology and the interethnic interaction, the various people promoting ethno-consolidation [3, page 57]. To possess ethno-cultural competence - means to recognize ethno-cultural pluralism, to have true, accurate knowledge of ethnic community and their culture, to understand their specifics. Ethno-cultural competence at the same time connects knowledge, ideas of ethnic community and their culture and the tolerant behavioral relations, which have been adjusted on interethnic mutual understanding and interaction. And in this plan formation of ethno-cultural competence is closed with education of ethnic tolerance.

The B.C.Gershunsky specifies, what exactly education is capable actively and to form purposefully mentality of tolerance [4, page 14] adheres to the current point of view also academician V.A.Tishkov who has put forward idea of pedagogics of tolerance which should be broadcast through education and cultural policy. 
On our concepts, tolerance is necessary for bringing up from preschool age when children only start to build the relations with each other. Education should go in a complex «kindergarten - school - higher education institution» in continuity of formation of bases of tolerance: tolerance concerning each other, understanding, interest and respect for traditions of other people living in the territory of the republic, and then, at learning of foreign languages, familiarizing with common cultural values and features of communication and culture of the people of other countries, education of friendship, training of intercultural communication.

\section{Bibliography}

1. Bibler, V.S.Kultura. Dialogue of cultures: experience of definition//philosophy Questions. - 1989. No. 6. - page 31-43

2. Sparrows, V. V. Lingvocultural science. Monograph / V.V.Vorobyev. - M: Prod. RUDN, 1997 $330 \mathrm{c}$.

3. Gershunsky B.S. Tolerance in system of valuable and target priorities of education//Pedagogics. 2002. No. 7, page 3-13.

4. Gurov, C.N, Vulfov, C.N, Galyapin, V.N. Formation of the tolerant personality in the polyethnic educational environment. Manual / V.N.Gurov, V.N.Vulfov, V.N.Galyapina. - М: Пед.общ. Russia, $2004-234 c$.

5. Lado, R. Lingvistics over borders of cultures. New in foreign linguistics/R. Lado. - Вып.25. М.:Прогресс, 1989. - page 32-62.

6. Safonova, V.P. Studying of languages of the international communication in a context of dialogue of cultures and civilizations. Safonov's monograph / Accusative - Voronezh: Sources, 1996 - 215c.

7. Singer M.R. Intercultural communication//Social sciences abroad. - 1989. - No. 2, page 80

8. Rierdon, B.E. Tolerance - the road to world / B.E.Rierdon - M: Prod. "Bonfi", 2001 - 302c.

9. Furmanova, V.P. Intercultural communication and a lingvocultural science in the theory and practice of training to Furmanov's foreign languages / Accusative. - Saransk: Prod. Pestilence. унив., 1993. - 120c. 